\title{
Human-in-the-Loop Segmentation of Earth Surface Imagery
}

This is a Preprint and has not been peer reviewed.

\section{Authors:}

D. Buscombe

Marda Science LLC, contracted to USGS Pacific Coastal and Marine Science Center, CA

E. B. Goldstein

Department of Geography, Environment, and Sustainability, University of North Carolina at Greensboro, NC

C.R. Sherwood

USGS Woods Hole Coastal and Marine Science Center, MA

C. Bodine

School of Informatics, Computing and Cyber Systems, Northern Arizona University, AZ

J.A. Brown

USGS MD-DE-DC Water Science Center, Dover, DE

J. Favela

Department of Earth and Planetary Sciences, University of California Santa Cruz, CA

S. Fitzpatrick

Department of Computer Science, California State University, CA

C.J. Kranenburg

USGS St. Petersburg Coastal and Marine Science Center, FL

J.R. Over

USGS Woods Hole Coastal and Marine Science Center, MA

A.C. Ritchie

USGS Pacific Coastal and Marine Science Center, CA 


\section{J.A. Warrick \\ USGS Pacific Coastal and Marine Science Center, CA \\ P. Wernette \\ USGS Pacific Coastal and Marine Science Center, CA}

Key points:

- Methodology for image segmentation based on agreement between a labeler and a machine learning model

- Faster more accurate segmentation of interpretable imagery compared to traditional labeling

- Large multi-labeler consensus facilitates reproducible scientific inference from Earth surface images

Version 1, Friday $15^{\text {th }}$ October, 2021

Associated software, Doodler: https://dbuscombe-usgs.github.io/dash_doodler/ 


\title{
Human-in-the-Loop Segmentation of Earth Surface Imagery
}

\author{
Daniel Buscombe ${ }^{1}$, Evan B. Goldstein ${ }^{2}$, Chris R. Sherwood ${ }^{3}$ Cameron. Bodine $^{4}$, \\ Jenna A. Brown ${ }^{5}$, Jaycee Favela ${ }^{6}$, Sharon Fitzpatrick ${ }^{7}$, Christine J. Kranenburg ${ }^{8}$, \\ Jin-Si R. Over ${ }^{3}$, Andy C. Ritchie ${ }^{9}$, Jonathan A. Warrick ${ }^{9}$ and Phil Wernette ${ }^{9}$ \\ ${ }^{1}$ Marda Science LLC, contracted to USGS Pacific Coastal and Marine Science Center,CA, USA \\ ${ }^{2}$ Department of Geography, Environment, and Sustainability, University of North Carolina at \\ Greensboro, NC, USA \\ ${ }^{3}$ USGS Woods Hole Coastal and Marine Science Center, MA, USA. \\ ${ }^{4}$ School of Informatics, Computing and Cyber Systems, Northern Arizona University, AZ, USA. \\ ${ }^{5}$ USGS MD-DE-DC Water Science Center, Dover, DE, USA. \\ ${ }^{6}$ Department of Earth and Planetary Sciences, University of California Santa Cruz, CA, USA. \\ ${ }^{7}$ Department of Computer Science, California State University, CA, USA. \\ ${ }^{8}$ USGS St. Petersburg Coastal and Marine Science Center, FL, USA. \\ ${ }^{9}$ USGS Pacific Coastal and Marine Science Center, CA, USA.
}

Friday $15^{\text {th }}$ October, 2021

\begin{abstract}
Segmentation, or the classification of pixels (grid cells) in imagery, is ubiquitously applied in the natural sciences. Manual methods are often prohibitively time-consuming, especially those images consisting of small objects and/or significant spatial heterogeneity of colors or textures. Labeling complicated regions of transition that in Earth surface imagery are represented by collections of mixed-pixels, -textures, and-spectral signatures, can be especially error-prone because it is difficult to reliably unmix, identify and delineate consistently. However, the success of supervised machine learning $(M L)$ approaches is entirely dependent on good label data. We describe a fast, semi-automated, method for interactive segmentation of $\mathrm{N}$-dimensional $(x, y, N)$ images into two-dimensional $(x, y)$ label images. It uses human-in-the-loop ML to achieve consensus between the labeler and a model in an iterative workflow. The technique is reproducible; the sequence of decisions made by human labeler and ML algorithms can be encoded to file, so the entire process can be played back and new outputs generated with alternative decisions and/or algorithms. We illustrate the scientific potential of segmentation of imagery of diverse settings and image types using six case studies from river, estuarine, and open coast environments. These photographic and non-photographic imagery consist of 1 and 3-bands on regular and irregular grids ranging from centimeters to tens of meters. We
\end{abstract}


Doodler: Human-in-the-Loop Segmentation of Earth Surface Imagery

demonstrate high levels of agreement in label images generated by several labelers on the same imagery, and make suggestions to achieve consensus and measure uncertainty, ideal for widespread application in training supervised ML for image segmentation.

Keywords - Machine Learning, Data Labeling, Interlabeler agreement, Gridded data, Earth surface processes, Geomorphology, Geospatial analysis and map creation

\section{Plain Language Summary}

Labeling pixels in scientific images by hand is time-consuming and error-prone, so we would like to train computers to do that for us. We can use automated techniques from Artificial Intelligence or AI, like one called Deep Learning, but it needs a lot of example images and corresponding labels that have been made by hand. So, we still need to label quite a lot of images at the pixel level —called image segmentation. We made a computer program called Doodler that speeds up the process; you label some pixels, and it labels the rest. It is the fastest method we know of for image segmentation because it is semi-automated. We also show that it produces accurate and precise labeling, as we demonstrated by having multiple people use this method to label the same images. Because it is so fast and accurate, it allows us to get enough data to train Deep Learning models to do segmentation on all the images we have, from the past and in the future. Doodler therefore enables geoscientists to use Artificial Intelligence to extract much more information from their imagery, in service of geoscience in general. 
Doodler: Human-in-the-Loop Segmentation of Earth Surface Imagery

\section{Introduction}

\subsection{The Need for Data Labeling Tools for Earth Surface Processes Research}

Automation of data-intensive tasks is increasingly important in Earth surface-processes research. Due to the availability of data at greater spatial and temporal coverages and resolutions [Farr et al. 2007, Gorelick et al., 2017, Wulder et al., 2019], and open-source geo-analytics tools [Schwanghart] and Scherler, 2014, Richardson et al. 2018], it is increasingly possible to automate the discovery of patterns in processes operating over complex landscapes [Walker et al., 2017, Larsen et al., 2021]. Scoping feasible applications of analytical tools such as machine learning (ML) in the geosciences has become a useful way to rapidly explore and prototype ideas with data [Reichstein et al., 2019. Goldstein et al., 2019].

Given the wealth of available ML algorithms in open-access software, geomorphologists have an unprecedented set of available tools for data exploration and hypothesis testing. Machine learning allows us to teach a computer to learn by example, usefully approximating quantities from readily obtainable data that are otherwise hard to sense [Buscombe et al., 2017], parameterize [Ni et al., 2021, Beuzen et al., 2019, Tinoco et al., 2015], flag for quality control [Sugiura and Hosoda, 2020], or to visualize or make automated inference on high-dimensional datasets that a human could not [Plant and Stockdon, 2012, Chmiel et al., 2021], especially for phenomena without well-developed theory [Fox et al., 2015, Goldstein and Coco, 2015]. However, the generation of the right type of examples for the machine to learn, or enough of sufficient quality, is a challenge that requires the development of specialist data labeling tools. These tools would allow Earth surface processes researchers to generate their own data representations for training ML to automate cleaning, distillation or classification of content, and make inference, on large geospatial datasets. 
Doodler: Human-in-the-Loop Segmentation of Earth Surface Imagery

An example is the segmentation of imagery.

\subsection{The Need for Better Tools for Image Segmentation}

What we hereafter call imagery is considered in the broadest sense as any dataset on a regular grid that may or not have a regular spatial footprint, which is collected for scientific applications in the Earth and environmental sciences and in related scientific fields. This definition includes geospatial datasets or rasters, photographic imagery, imagery from satellites, sonar, radar, and other geophysical sensors, and any other gridded data that is visually interpretable (by a subject matter expert or otherwise). Such Earth surface imagery comes in a range of types, from single-band or greyscale commonly created by sensors used in geophysical applications that consist of interpretable textures and edges, to hyperspectral imagery where up to hundreds of coincident bands sense a different narrow portion of the electromagnetic spectrum. We use the term pixels to mean either pixels or voxels, depending on whether the imagery is two- or three-dimensional.

The increasing availability of imagery and increasing acceptance [Olhede and Wolfe, 2018], accessibility [Gil et al. 2016], and sophistication of human-supervised computerized analyses and classification workflows [Cheng et al., 2001, Hossain and Chen, 2019, Mi and Chen, 2020], mean that accurate image segmentation workflows - involving the classification of all pixels in an image - are ubiquitous in need and application in the geosciences [Carleer et al. 2005, Kotaridis and Lazaridou, 2021]. Probabilistic segmentation of imagery using ML has various uses in Earth surface processes research [Lang et al., 2019] involving environmental monitoring [Anders et al., 2011, Gaddes et al., 2019, Bayr and Puschmann, 2019, Su et al., 2020]. Detection of change in geomorphic studies has traditionally involved differencing of elevation surfaces [James et al., 2012]. Segmentation of coincident imagery allows for additional insight, for example the classification/attribution of the change, evaluation of the agent of change [Grams et al., 2019], the nature and persistence of change, 
Doodler: Human-in-the-Loop Segmentation of Earth Surface Imagery

and determination of implications [Barlow et al., 2006, Drăguţ and Eisank, 2012]. Understanding these insights is key to habitat monitoring [Ridge et al., 2019, Chilson et al., 2019, Gray et al., 2019] and land use or cover (change) mapping [Lefsky, 2010, Buscombe and Ritchie, 2018, Carbonneau et al. 2020, Pandey et al. 2021] among many other examples [Weinstein, 2018, Chaudhary et al., 2019, Quinn et al., 2018, Ching et al., 2018].

State-of-the-art ML-based image segmentation requires at least some level of human supervision [Kotaridis and Lazaridou, 2021, Sultana et al., 2020]. Often the greatest challenge to developing an automated workflow can be the creation of model training data that is internally consistent [Serre, 2019]. In the case of image segmentation, training data consists of label imagery where each pixel is categorized into any number of pre-determined discrete nominal or ordinal classes. Many applications of segmentation of Earth surface imagery by definition are concerned with surfaces, therefore the focus of many labeling workflows, and also the present contribution, is the generation of 2D label images by segmenting visually interpretable imagery, i.e., up to three coincident bands.

Such label imagery is typically acquired by either hand-digitizing vector polygons that are subsequently rasterized [Kotaridis and Lazaridou, 2021], or raster editing, which is hand classification of pixels directly. Creating label images through digitization of hand-drawn polygons is time-consuming; raster-editing can offer a quicker alternative, and most commercial and noncommercial image-editing software also have built-in tools that can select entire regions via similar colors or edge-detection techniques. These tools are typically a) not reproducible because the outputs are generated by a sequence of clicks that are not recorded in a file (a fact that precludes many of the analyses of multi-labeler agreement we present here), b) proprietary or restrictively licensed, and/or c) still require significant amounts of time and effort to achieve good results. The largest error is at boundaries between classes, and arises due to two factors: a) indistinct areas of 
Doodler: Human-in-the-Loop Segmentation of Earth Surface Imagery

transition where it is not always possible to make an objective decision about the class, and b) it is almost never feasible to click the shape of a polygon outline at the pixel level.

Labeling Earth surface imagery using these traditional methods is especially time-consuming if images consist of small or unfamiliar objects and/or colors or textures exhibiting significant spatial heterogeneity and/or ambiguity, necessitating a high zoom level, or viewing at a range of scales. Moreover, labeling transition regions is difficult to do reliably because of mixed-pixels, -textures, and -spectral signatures, which can lead to significant amounts of error. Earth surface imagery is more likely to have these properties than much imagery used to develop image segmentation models, labeling tools, and benchmark datasets in ML research and applications [Everingham et al., 2010]. In Earth surface imagery, and especially in transition areas, we argue that pixelwise classification needs a human for these transition regions and more complex textures, but could also be sped up by including techniques that aid the human labeler, such as ML models that are trained as a human annotates.

\subsection{Human-in-the-Loop Image Segmentation}

Here we describe and evaluate a so-called 'human-in-the-loop' [Monarch, 2021] machine learning workflow for fast image segmentation, encoded in a computer program called Doodler, and we demonstrate its use for geophysical, photographic, and multispectral satellite images of natural environments. Doodler lies on the spectrum of what Monarch [2021] refers to as 'assisted annotation,' which is interaction with raw data, with ML assisting the data labeling process, and 'predictive annotation,' where ML generates outputs that can be edited. In fact, the program essentially does both, in a loop whose number of iterations for any given sample is dictated by the human labeler who acts to assure data quality.

As supervised ML workflows gain popularity in the geosciences [Bergen et al., 2019, Zuo 
et al., 2019] and related fields [Crisci et al., 2012, Kashinath et al., 2021a], Doodler could be used in numerous contexts to reach a target ML model accuracy by training on large amounts of data acquired relatively quickly. It also serves as a case study in how to combine human and machine intelligence to label scientific data with increased efficiency and accuracy. In the next section we introduce the human-in-the-loop labeling principles and graphical (in the sense of Koller and Friedman [2009] of models consisting of nodes connected by vertices) model framework, followed by a description of the image feature-extraction methods, and the ML classifier. In section 3 we describe six datasets that we use to demonstrate the approach. These are chosen to quantify and discuss variability among label images made by several independent labelers, and further to examine variability in image segmentation outputs due to image size and resolution.

Comparisons between images labeled by the same labeler at different scales, and multiple labelers of the same imagery are presented in section 4 . This section serves a few purposes. First, for subjective tasks involving interpretation of ambiguous data, or even objective tasks or relatively simple tasks where random human blunder may be a factor, no simple heuristics exist for deciding the correct label [Monarch, 2021] however some practical recommendations can be made using statistical metrics of multi-labeled datasets [Goldstein et al.,2021]. Similarly, we offer some methods for identifying and quantifying uncertainty based on agreement over segmentations of the same imagery by multiple labelers. Second, this section serves to demonstrate that the methodology and implementation we present are reproducible between labelers, at different times, and using different computational infrastructure (computers, browsers, etc.), despite the fact that the label image is a model estimate from sparse annotations that would vary considerably from labeler to labeler. In section 5 we make suggestions on how to achieve consensus and measure error, and recommendations over usage of the Doodler program, before drawing conclusions. 
Doodler: Human-in-the-Loop Segmentation of Earth Surface Imagery

\section{Human-in-the-Loop Labeling using Machine Learning}

The image labeling task (Figure 1) involves a human labeler providing sparse annotations (informally called 'doodles') to inform and automate a process ('model') that estimates the label for all pixels in that image, then the same labeler refine the model predictions using a combination of adding/removing doodles and/or changing model hyperparameter values. A workable system necessitates a graphical user interface and a fast and accurate image segmentation process. Each image is classified according to a set of pre-determined classes; we use the term label to refer to a single instance of an annotation of a specific class, such that each class present in every image is exemplified with numerous labels.

The images are segmented semi-interactively, one-by-one, so there is no need to specify an underlying prior statistical model, and we need not assume pixel values are conditionally independent of a given label. Therefore ML is ideally suited to the task; because it could learn how to map the features that may be readily extracted from imagery, to class labels, from a small proportion of labeled pixels. That model could then be used to estimate the class of the remaining pixels not labeled. More formally, we use a discriminative ML model, $f$, that has learned the conditional distribution $P(y \mid \theta, x)$ directly, which reads as the probability of $y$, given $\theta$ and $x$, where $x$ are the image features associated with annotated pixels $y$, and $\theta$ are learned parameters. This approach is highly suited to task-specific prediction such as here; the models need not be portable among images, therefore no attempt is made to capture the distributions over $x$ or model the correlations among $x$. The model then predicts the class $\hat{y}$ of the unlabeled pixels $\hat{x}$ by $\hat{y}=f(\hat{x})$, essentially by assuming $P(\hat{y} \mid \theta, \hat{x}) \approx P(y \mid \theta, x)$.

The system consists of 1) a human annotator providing sparse examples of each class of interest in a graphical user interface running in a web browser, 2) a Multi-Layer Perceptron (MLP) 
model [Bishop, 2006] for per-pixel class, based on a probabilistic model of how classes relate to a stack features extracted from standardized imagery based on intensity, texture, edges, and relative location, controlled by parameters learned during a discrete training period, and 3) a graphical model called a fully connected conditional random field (CRF) [Kumar and Hebert, 2006] that refines estimates of the per-pixel class based on a probabilistic assessment of how classes relate to features extracted from imagery based on both color (if 3 or more dimensions) or intensity (if imagery is 2D) and relative location, controlled by hyperparameters set/tuned by the human labeler, who also acts to assess quality, and iterate as needed. We use the two ML models in conjunction with human annotations to classify each pixel of the scene and segment the image. At least one of the classes must exist in a given image, but otherwise there are no restrictions on the number of classes (other than practical considerations such as available time). Often models need the most detailed annotations or 'doodles' near the boundaries where one class transitions to another.

The program facilitates human labeling, which also provides quality control. In effect, the labeler interacts with a machine to collectively decide on the most accurate and precise label image for any given image. Doodles are used to update the ML model iteratively, by adding/removing annotations, and also optionally changing hyperparameters for optimal segmentation on individual images, and retraining and implementing the model. The program relies on the labeler having the patience, dedication, and interest to do a good job, which may require a few iterations of the workflow (Figure 11). The design of the program would also be amenable to labeling in stages, with each stage perhaps employing people with different levels of expertise. We now describe the two ML models embedded within the doodler workflow, namely the Conditional Random Field (2.1) that uses a Multilayer Perceptron or MLP (2.3) as a sub-component. We conclude by describing our implementation of the Doodler workflow in 2.4

The methodology not only facilitates much faster segmentation, which makes multiple labeler 
Doodler: Human-in-the-Loop Segmentation of Earth Surface Imagery

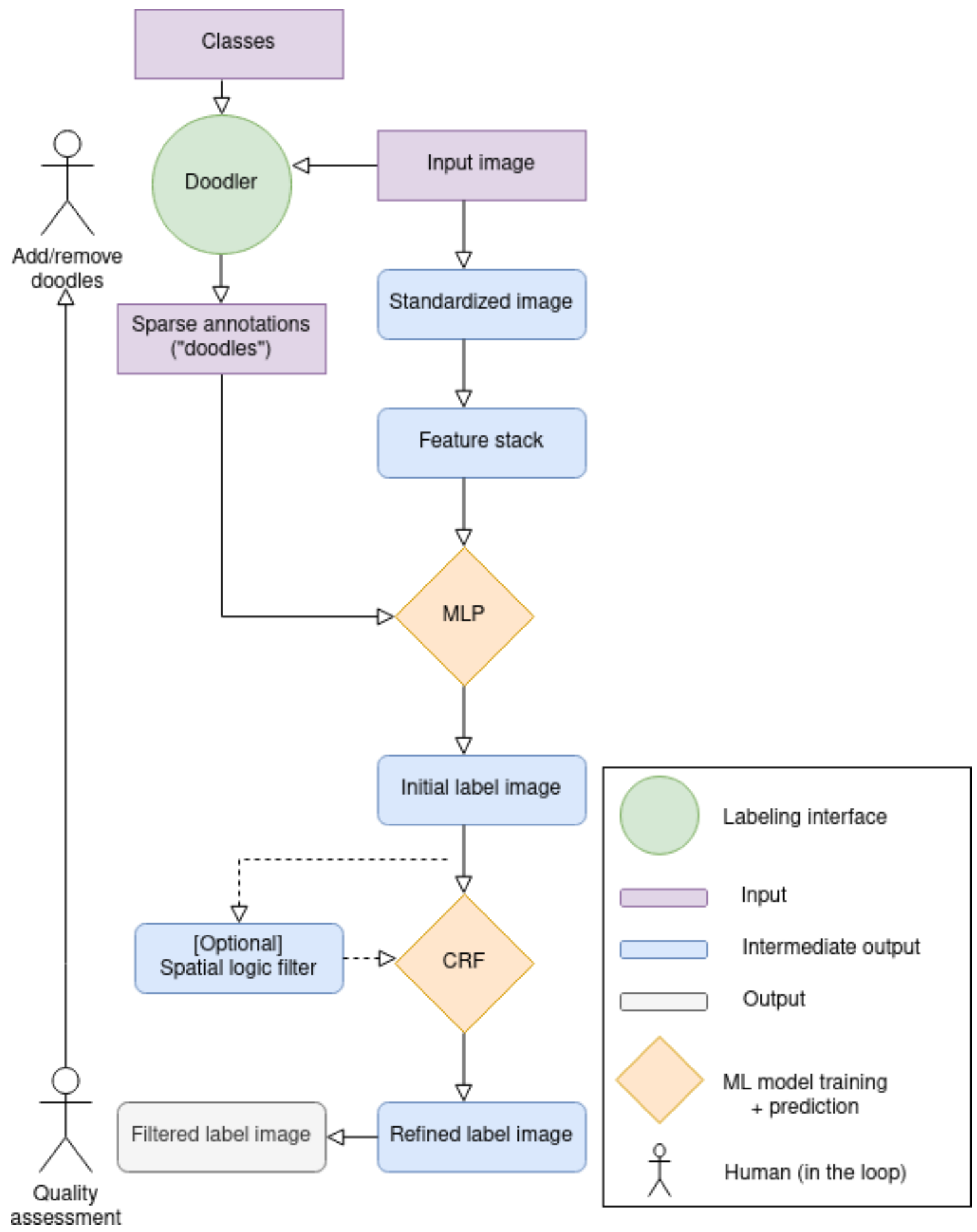

Figure 1: Schematic of the approach encoded into the Doodler program. Most images-class set pairings trialed to date have been segmented successfully within one or two loops. Doodler also facilitates the user to modify the model hyperparameters that may be used iteratively the same way as adding or removing annotations ('doodles'). 1 The human adjusts the hyperparameters and they feed into the Multilayer Perceptron (MLP) and Conditional Random Field (CRF) models. 
Doodler: Human-in-the-Loop Segmentation of Earth Surface Imagery

datasets more obtainable (affordable, and completed in a reasonable time), but also results in more accurate segmentations. That is because the labeler is asked only to provide true and unambiguous positive examples of each class. Errors at boundaries between classes that arise due to hand digitization, which can be significant because of mixed pixels or due to coarse digitization, are significantly reduced. That is because the program predicts at the pixel level much faster than a human could ever label at that scale, and also because our approach models the likelihood of uncertain regions. The latter is crucially important for class assignment in particularly difficult regions of imagery in a deterministic manner.

\subsection{Conditional Random Field for Image Segmentation}

We adopt a widely used approach to such task-specific probabilistic image segmentation, which is a Conditional Random Field or CRF model [Kumar and Hebert, 2006, Zhong et al., 2014, Vosselman et al., 2017] to estimate per-pixel class likelihoods (Figure 2). We use the similar CRF implementation of Krähenbühl and Koltun [2011] that was previously used by Buscombe and Ritchie [2018]. Whereas Buscombe and Ritchie [2018] used a trained convolutional network to label regions of images that were used as unary potentials for a CRF model for pixel-level refinement, and Buscombe and Grams [2018] used sparse instrumental observations from the field in conjunction with geospatial imagery, here (Figure 2) labels of some regions of images are provided by humans, which are used to ascribe a probability of each class per pixel using a Multilayer Perceptron. Those outputs (per-pixel class likelihoods) are used as unary potentials for a CRF model for pixel-level refinement; the CRF model additionally models the joint likelihood of each pair of pixels, essentially checking for internal consistency of the MLP outputs.

The unary potentials define a log-likelihood over the label assignment $y$, and therefore represent the cost of assigning label $y_{\mathbf{i}}$ to grid node $\mathbf{i}$. They are called 'unary' potentials because 
they describe feature-class relations at every pixel, and to distinguish them from pairwise potentials, dependent on feature-class relations over pairs of feature-class relations, which are also used in the CRF model and defined later. Here we use a Multilayer Perceptron [Bishop, 2006] as a classifier to generate unary potentials. In CRFs based on 'local' connectivity, nodes connect adjacent pixels in $x$ [Kumar and Hebert, 2006], whereas in the fully connected definition such as here (Figure 2f), each node is linked to every other [Krähenbühl and Koltun, 2011]. Linking each node of the graph created from $x$ to every other enables modeling of the long-range connections within the data by considering both proximal and distal pairs of nodes, resulting in refined labeling at boundaries and transitions between different classes. We use a global probability prior $p_{u}$ of the unary potentials, i.e., a prior probability that any random sample correctly labels the underlying image features. It is exposed to the user as a seldom-varied hyperparameter, defaults to 0.9 , and generally has limited effect unless provided annotations are actually of poor quality, which we assume is rarely the case.

There are two non-dimensional hyperparameters exposed to labelers using the Doodler program. The first is $\theta_{\beta}$ (default $=1$ ) is used by the $\mathrm{CRF}$ feature extractor to extract color image features and map them to classes. These features are engineered, by convolving Gaussian kernels with the imagery (in much the same way as features are extracted as inputs to the MLP model -see section 2.2). Hyperparameter $\theta_{\beta}$ controls the degree of allowable similarity in image features among classes, therefore $\theta_{\beta}=1$ only tolerates image features with small differences in intensity being assigned the same class label.

a The second hyperparameter, $\mu$, is used within a Potts label 'compatibility' function Krähenbühl and Koltun, 2011] to define pairwise potentials used by the model to encourage adjacent pixels to be the same class label, defined as $\Lambda(i, j)=\mu$ if $i=j$ and 0 otherwise. By default, Doodler uses $\mu=1$, meaning $\Lambda$ is simply a $k \times k$ identity matrix, whereby all classes are equally 'compatible' (as likely as each other to be adjacent in either image or feature space). Values greater than 1 weight the 


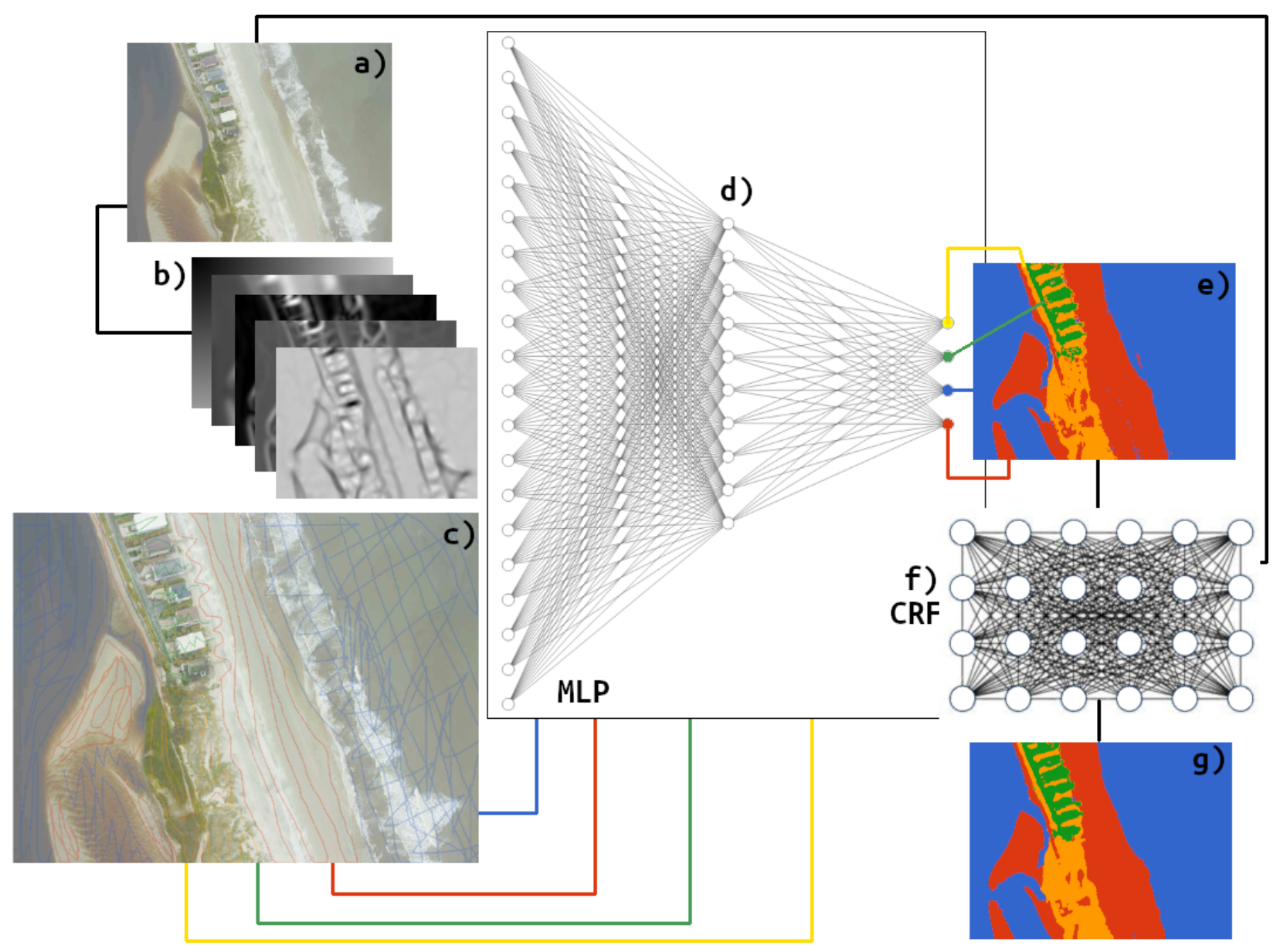

Figure 2: An illustration of how image data (a) are used to extract features (b) that are used in conjunction with sparse annotations (c) to train an initial Multilayer Perceptron (MLP) classifier (d) to extract unary potentials (e) that are refined by a Conditional Random Field (CRF) (f) to create a refined label image $(\mathrm{g})$. 
pairwise potentials more than the unary potentials, which might be useful when the MLP prediction is poor, in which case the pairwise potentials count by a factor of $\mu$ greater than the unary potentials. By definition, $\theta_{\beta}$ and $\mu$ are task-specific, so their respective effects are hard to generalize, but it can be said that, in general, larger values of $\mu$ tend to give the model greater independence, resulting in the reclassification of more pixels. The importance of pairwise potentials becomes much greater than unary potentials, and spatial inconsistencies in feature-label pairings have greater likelihood of being reclassified. In general, $\theta_{\beta}$ has a more muted effect and generally controls the sharpness of the class boundaries in the label image. Note that neither effect necessarily improves the result. Please refer to Figure $\mathrm{S} 1$ for visualizations of the effects of varying $\theta_{\beta}$ and $\mu$ on sample imagery from the Sandwich dataset, expressed in terms of where the labels of pixels are altered by the CRF compared to the MLP output. The reader is also referred to the Supporting Information section entitled 'Fully Connected Conditional Random Field for Image Segmentation' for more technical details about its implementation and interpretation of parameters.

By design, the CRF solution is not overly sensitive to hyperparameter values. First, imagery is standardized therefore the model does not need to use parameters for brightness (related to non-zero image mean) and contrast (related to non-unit image variance). Second, we use spatial logic to filter CRF inputs, which eliminates a major source of uncertainty for the CRF solution employing pairwise potentials, because the CRF model will be given more consistent spatial pairs of feature-class-pairings to make inference from. Finally, hyperparameter sensitivity increases if the sparse annotations are used alone [Buscombe and Grams, 2018], and/or if the unary potentials estimated by the MLP model are spatially sparse [Buscombe and Ritchie, 2018]. 
Doodler: Human-in-the-Loop Segmentation of Earth Surface Imagery

266

267

\subsection{Image Standardization and Feature Extraction}

Each input image, $I(i, j, d)$, where $i$ and $j$ describe 2D pixel locations and $d$ indicates the number of coincident data layers, is standardized such that it has zero mean and unit variance (see Supporting Information section entitled 'Image Standardization and Feature Extraction'). This ensures the values are distributed within the range -1 and 1 , which helps numerical stability and builds insensitivity to outliers, as well as removing any bias from any channel as a function of the mean image intensity. Raw pixel values are not used as inputs to the MLP classification model described in section 2.3. Instead, features are extracted in a prescribed way i.e., the image features are extracted in the same way each time, known as feature engineering. Features relating to image intensity, edges, texture, and relative location are extracted, all at a range of scales. Then a stack of features are provided to the classifier. We use kernel convolution methods for feature extraction because they are already common in numerous geophysical applications concerning interpretation and quantification of spatially distributed imagery. Image intensity features $I_{f}(i, j)$ are extracted from $I_{s}(i, j, d)$ by convolving with filter bank $\Sigma_{s}$, or $I_{f}=\Sigma_{s} * I_{s}$ where $*$ denotes convolution, and where $\Sigma_{s}$ consists of $s$ 2D Gaussian kernels.

Edge features are extracted using the Sobel operator, computing an approximation of the gradient magnitude of $I_{f}, \nabla_{I_{f}}(i, j)$. Location is encoded as the kernel-convolved bank of $2 \mathrm{D}$ features given by $\left.L(i, j)=\Sigma_{s} * \sqrt{(} i^{2}+j^{2}\right)$. Finally, texture features are computed as the first and second eigenvalues of Hessian matrix of $I_{f}(i, j)$, or $H_{1}(i, j)$ and $H_{2}(i, j)$. Eigenvalue analysis of the Hessian is commonly used in geophysical and medical image feature-extraction [Bishop, 2006] because of its formalized relationship to physical quantities, extracting the principal directions in which the local second order structure of the image, i.e., its spatial covariance structure, can be decomposed. The eigenvectors and eigenvalues of the Hessian are known as principal directions 
Doodler: Human-in-the-Loop Segmentation of Earth Surface Imagery

and principal curvatures respectively [Koenderink and Van Doorn, 1992]. The first two eigenvalues are the magnitudes of the maximum and minimum curvature, respectively.

\subsection{Initial Segmentation Using a Multilayer Perceptron}

The feature stack used for initial segmentation consists of a set of 3D $(i, j, d)$ grids, each flattened to $1 \mathrm{D}(1, i j d)$, then stacked columnwise to create a model input vector. The feature stack is then subsampled row-wise by a factor defined by the user. For larger imagery, this subsampling factor may be as large as six, but typically it is one (i.e., no subsampling) to three, and depends on the available computer memory and processing time.

Our entire model framework implementation (see Supporting Information section entitled 'Multilayer Perceptron') consists of an input layer of $i j d$ neurons, two hidden layers, the first consisting of 100 neurons and the second of 60 neurons, each linked to each other (i.e., fully connected), and finally a classifying layer consisting of $k$ neurons, where $k$ is the set of classes with labels, i.e., present in the scene, determined a priori for the scene. Through extensive experimentation, we are satisified that model outputs are not overly sensitive to the specification of the number of neurons in each of the two hidden layers. However, hidden layers or neurons could be added for greater discriminative power at the expense of model parsimony and computational efficiency. MLPs have previously been successfully used for Earth surface image segmentation [Kurnaz et al., 2005, Villmann et al. 2003], as have other types of artificial neural networks [Kemker et al., 2018, Buscombe and Ritchie, 2018].

While any number of similar deterministic ML algorithms could have been used, MLPs are attractive due to their relative simplicity and longevity which has created a widespread use of them among many geoscience and related fields [Gardner and Dorling, 1998]. Because this is task-specific prediction, $90 \%$ of the input feature data are used for training and only $10 \%$ for validation, for 
Doodler: Human-in-the-Loop Segmentation of Earth Surface Imagery

iteratively adjusting $\mathbf{w}$ and $\mathbf{b}$ through back-propagation and solved using stochastic gradient descent, with a maximum of 2000 training epochs. We use the Adam stochastic gradient-based optimizer method proposed by Kingma and Ba [2014], using early stopping to terminate training when the validation score does not improve by $1 \mathrm{e}^{-4}$ for at least 10 consecutive training epochs. Model outputs are not very sensitive to hyperparameters, i.e., choices about percentage of data used for validatation, number of training epochs, or criteria for terminating the training. For brevity, this sensitivity analysis is not shown here but the program documentation explains where these hyperparameters may be adjusted and their resulting outputs compared.

Whereas there is no drop-in replacement for the CRF, the MLP could be switched to a different ML framework. In fact, we have also extensively trialled a Random Forest model framework but decided that the MLP performed better; see Figure S2 for an example, based on dataset A.

\subsection{Implementation: The Doodler Program}

In a human-in-the-loop data labeling system, the design of the front-end annotation interface is as or more important than the back-end ML model framework. At a minimum, the user interface must allow for image annotation and a mechanism for launching the image segmentation process (Figure 3). Optionally, it can also expose controls to facilitate image curation and class (label) definition, mechanisms to adjust hyperparameters, and controls for re-segmentation. We have created several versions of the program, including some that store images locally, and others that retrieve imagery from a remote server. The latter case is useful for collaborative labeling projects, because the application can be hosted on the worldwide web and the results can be stored centrally.

The default version of the program that we have made publicly available allows the user to place images for classification in a local 'assets' folder. The program tracks images that have been classified, therefore the list of files available for classification gets smaller during a labelling session. 
Users can also modify hyperparameters and redo segmentations as many times as desired, as well as the 'pen' width (width in pixels to ascribe each annotation). These controls can optionally be hidden from the user in order to only collect the sparse annotations, and/or (pixelwise) label images with a fixed set of default hyperparameters.

Each MLP prediction is a matrix of dimension $i j k$ encoding the probabilities of each pixel $i, j$ and each class $k$. The discrete class is found as the maximum over $i, j$ in the $k$ dimension, or argmax, resulting in a label matrix of integer values, each integer corresponding to a unique class. Often there can be high-frequency noise in the resulting 2D discrete label image of pixelwise predictions, i.e., small islands of misclassified pixels. Since classifer outputs are probabilistic, instead of using argmax we could choose to filter these islands based on logic or some other process operating on the probabilities themselves, or we could filter islands by operating in the spatial domain on the label image. Doodler implements the latter, using two complementary filtering procedures. Therefore we implement an additional, but optional, step is performed in which the label matrix output from the MLP model is spatially filtered. The filtered label is then used as input to the CRF model. The reader is referred to Supporting Information section 'Spatial Filtering of Initial Segmentation' for more details. An illustration of the full workflow described in sections 2.1 through to the present section, including the spatial filtering of the initial segmentation, is presented as Figure S3.

\subsection{Comparison of Segmentations}

In order to quantify inter-labeler differences, the canonical metric to evaluate the difference between two thematic maps or label images [Costa et al. 2018] is the mean Intersection over Union score (IOU, or Jaccard Index) averaged over $k$ classes. For a collection of overlapping regular shapes, an IOU value of 0.5 would imply average overlapping by $50 \%$, but in this context contributions are summed over fields, therefore when IOU reflects $50 \%$ average overlap between each contiguous 
Doodler: Human-in-the-Loop Segmentation of Earth Surface Imagery
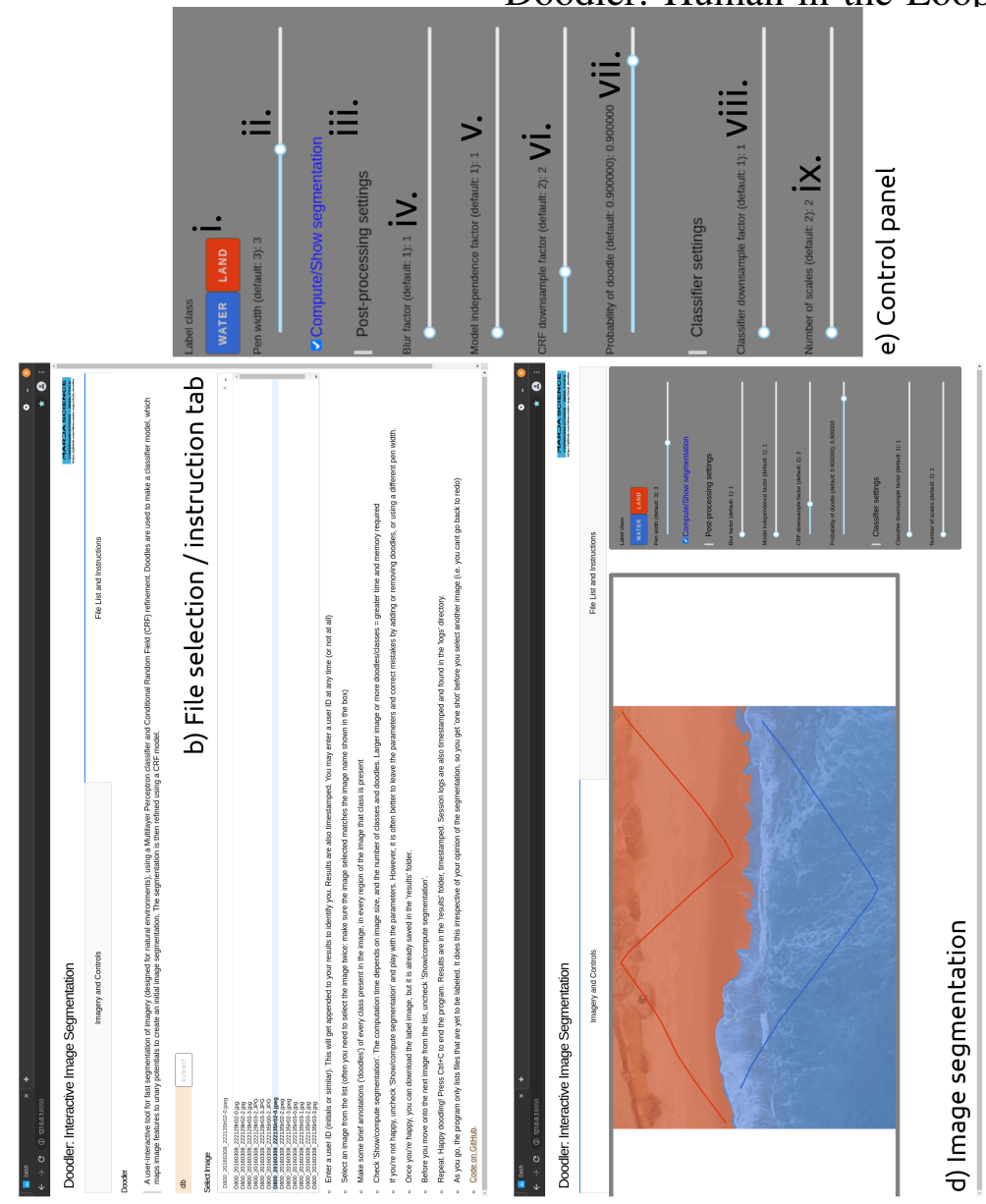

สิ

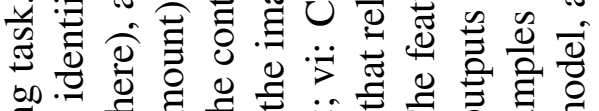
.0

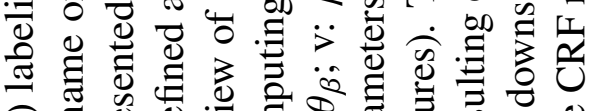

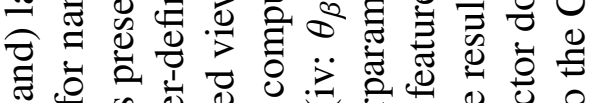

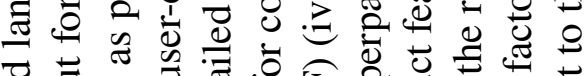

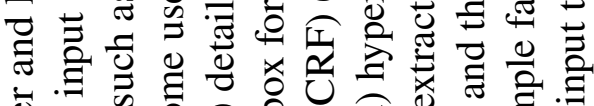

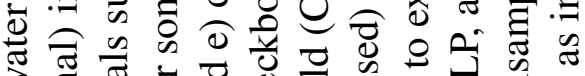

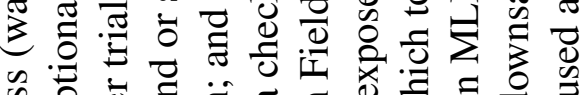

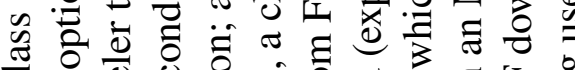

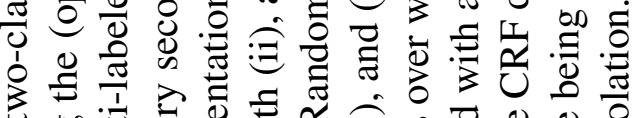
on

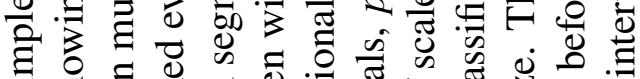

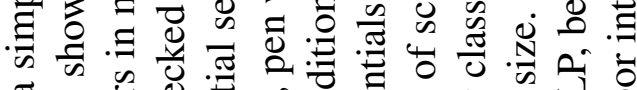
๘)

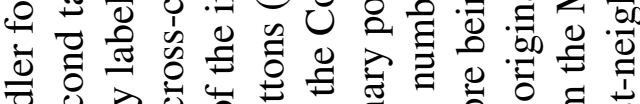
ᄋ

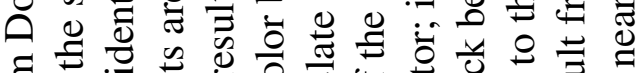
छ

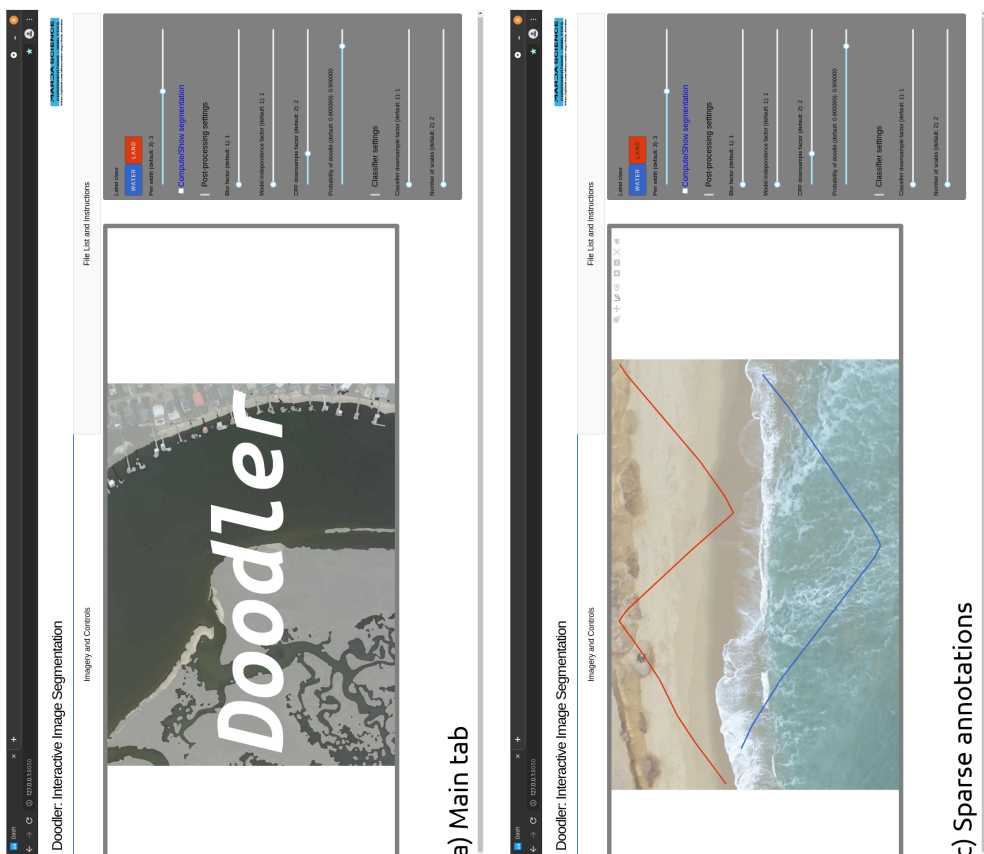

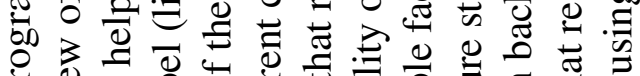

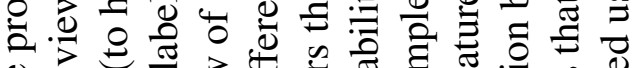

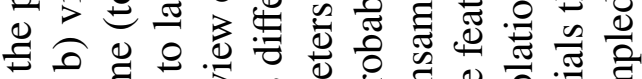

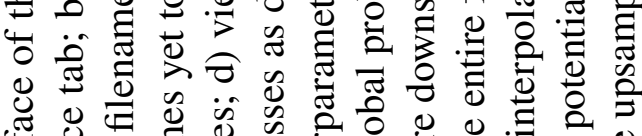

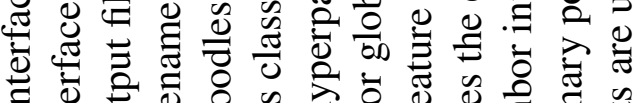
.

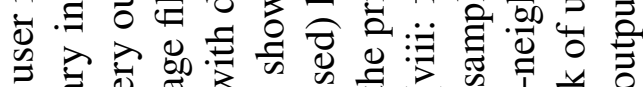
శ్ 承

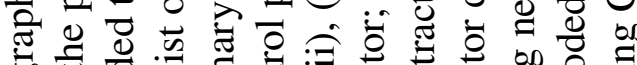

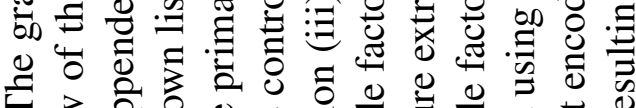

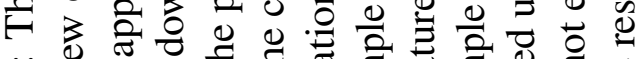

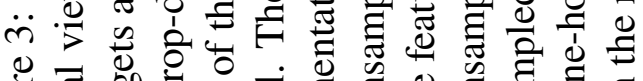

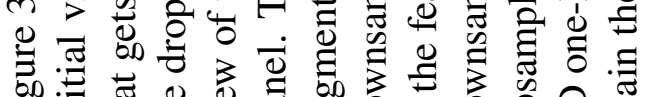

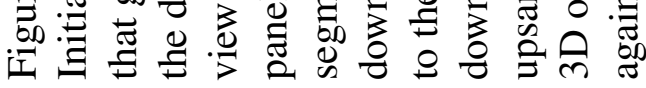


region of labeled pixels. However, many label images are class-imbalanced, which is to say there tends to be a majority class and one or more minority classes.

The mean Dice score is relatively insensitive to the number of pixels total in each class, because the numerator is the number of correctly classified pixels, and the denominator is the total number of pixels in a class that is in both estimated and observed. It has therefore been suggested to be a more accurate metric for the overall agreement between two label images for class-imbalanced label images, whereas an IOU score is not as sensitive to contributions from the smaller class [Csurka et al. 2004]. The reader is referred to Figure S4 for the functional relationship between mean Dice and mean Intersection over Union, and to Figure S5 for an illustration of the behavior of these metrics for a sample comparison using one dataset, and to the Supporting Information section entitled 'Comparison of Segmentations' for mathematical details about the two metrics.

For both IOU and Dice scores, where different numbers of unique classes exist, i.e., two different candidates for $k$, we could choose to set $k$ as the minimum number of the two respective class sets, or the maximum number. We chose the maximum, therefore scores are conservative in these situations. It might be surmised that Dice measures average accuracy, while IoU measures something closer to the worst-case accuracy. However, they vary nonlinearly and, due to averaging over classes, exhibit independently useful properties. We present both scores for each dataset, and also use them to discuss ways to detect class imbalance, outlier labelers, and label images in multi-labeler contexts, as well as reporting mean agreement for multi-labeled datasets as an uncertainty and quality metric. 
Doodler: Human-in-the-Loop Segmentation of Earth Surface Imagery

\section{Datasets and Case Studies}

We demonstrate our approach using several case studies from riverine, estuarine, and coastal environments of the United States, chosen to illustrate the scientific potential of image segmentation in diverse environments and image types, and more specifically to quantify inter-labeler-agreement under various contexts. The datasets (Table 1) consist of one- and three-band imagery on regular and irregular grids ranging from centimeters to tens of meters, including photographic and nonphotographic imagery. Segmentation of this imagery can be used to answer a range of scientific questions concerning landscape change, which we exemplify for each dataset below. In each case, the labelers were issued instructions only verbally, rather than demonstrating with examples. The task was discussed, then attempted once and not redone.

\subsection{Sedimentary Mapping of a Mixed-Sand-Gravel Beach from Visible-Band Aerial Orthomosaic Imagery}

Dataset A [Sherwood et al., 2021] consists of one, three-band orthomosaic image (Figure 4a, Table 11], at $5-\mathrm{cm}$ and also downsampled to a resolution of 25-cm, for mapping beach substrates of Sandwich Town Neck Beach on Cape Cod, Massachusetts. The orthomosaics are created from photographs collected from a low-altitude Uncrewed Aircraft System (UAS) on September 21, 2016, using a structure-from-motion workflow similar to that described by Over et al. [2021] for high-resolution elevation mapping of coasts from aerial imagery [Warrick et al., 2019]. The 5-cm and 25-cm pixel imagery are divided into 1024 x 1024 pixel, 3-band (RGB) tiles for annotation, which results in 99 and six tiles for the respective resolutions. The two datasets were labeled by different individuals. The reader is referred to Figures $\mathrm{S} 1,2$, and 3 for more example imagery. The following categories are used; 1) water, 2) sand, 3) gravel, 4) cobble/boulder, 5) vegetated, 6) development. 


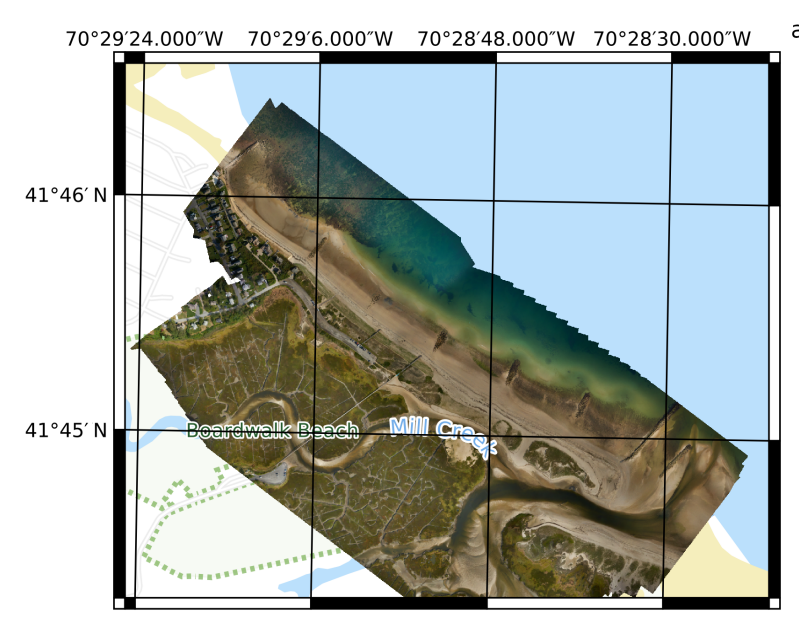

a) Dataset $A$

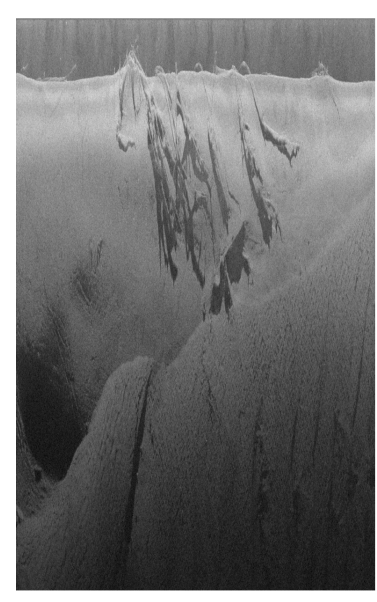

d) Dataset D

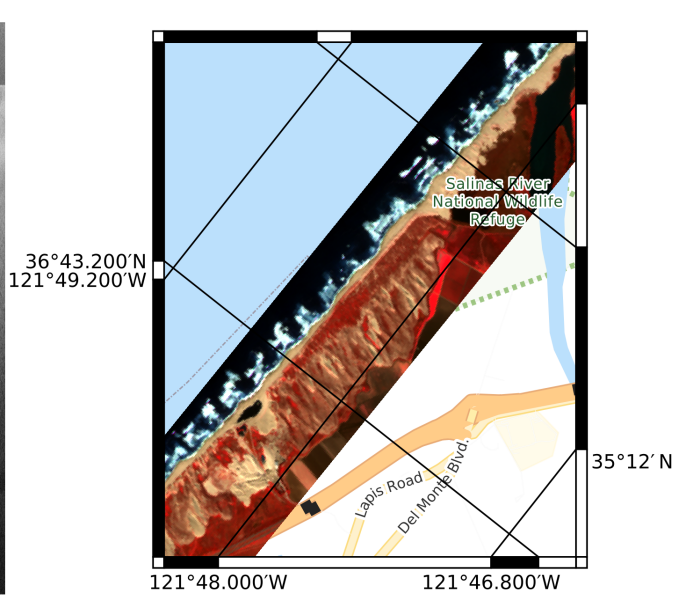

e) Dataset E

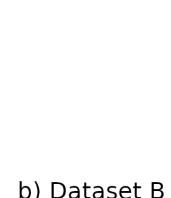

b) Dataset B

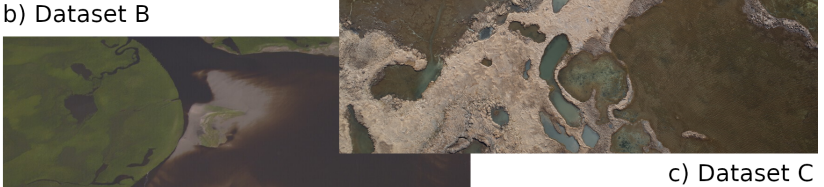

c) Dataset C

Figure 4: One example image from each of the six datasets used in this study, from left to right; a) a portion of an orthomosaic image of a beach, b) an aerial image of a marsh environment, c) an aerial image of a backbarrier coastal dune environment, d) a portion of a sidescan echogram from a coastal plain river, and e) a false-color multispectral satellite image of a coastal lagoon and vicinity. Geospatial imagery on regular grids are shown with latitude and longitude grids and labels. 
Doodler: Human-in-the-Loop Segmentation of Earth Surface Imagery

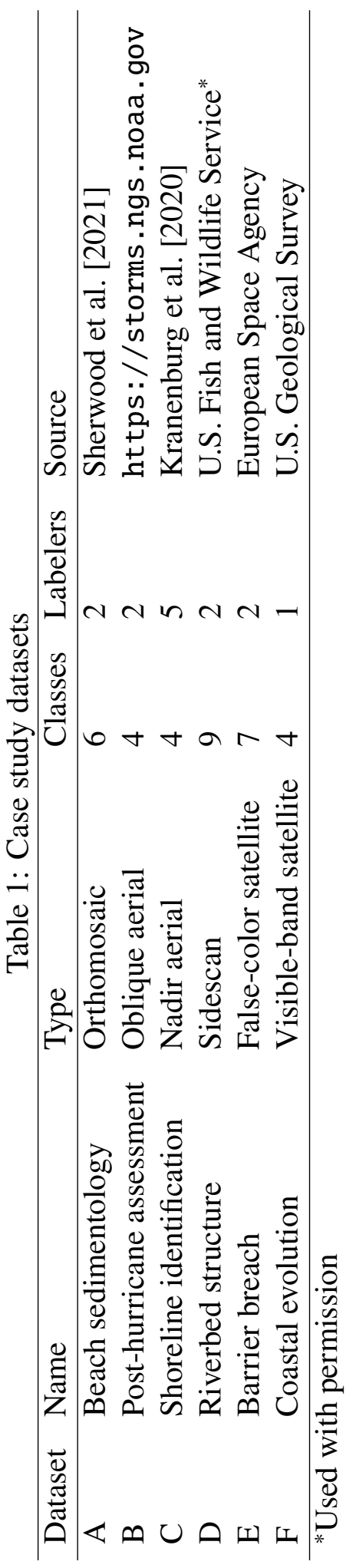


Doodler: Human-in-the-Loop Segmentation of Earth Surface Imagery

The orthomosaics are used to evaluate the products resulting from labeling images at two resolutions. They are also used to illustrate how to determine optimal image and pixel size for annotation. Such imagery is used for tracking changes to the beach morphology and sedimentology, such as tracking the position of the shoreline, berm, and scarp to indicate the nature of morphological change, as well as individual sediment fractions such as gravel patches that may have a morphodynamic role or could be sensitive coastal state indicators. Segmentation is also useful for determining which parts of the scene are usable data for subsequent analyses. In some situations when working with large imagery, it is difficult to know a priori what image size to use when annotating using the methods described here; while the program facilitates zooming and panning (see section 2.4 for details on our program implementation), sometimes it is more efficient to use smaller image tiles. In other situations, there is a choice over what grid size to use when making the imagery, such as when converting from ungridded to gridded data. The orthomosaics are created from color-attributed 3D point clouds [Over et al., 2021], therefore we use dataset A (Table 1) to discuss a workflow designed to experimentally determine optimal grid size and image size ahead of a large labeling task.

\subsection{Flood Detection in Post-Hurricane Aerial Photographic Imagery}

Dataset B (Figure 4b, Table 1) consists of a non-continuous spatial series of 80, three-band image tiles ( $1000 \times 750$ x 3 pixels), which are from Emergency Response Imagery collected by the National Geodetic Survey Remote Sensing Division of the US National Oceanographic and Atmospheric Administration, NOAA, [NOAA, 2021] that have been each divided into four tiles. The imagery is from North and South Carolina taken after Hurricane Florence (2018). Post storm imagery can be used to monitor the effects of hurricanes on coastal communities [Chen et al. 2018] and ecosystems [Barnard et al. 2021] and coastal change [Goldstein et al., 2020]. The images are labeled using the 
following classes: 1) water, 2) sand, 3) vegetated surface, and 4) development. We compare the segmentations from two labelers labeling the same complex imagery that is readily interpretable without specialist knowledge, but nevertheless difficult to interpret all classes consistently. The reader is referred to Figure S6 for more example imagery.

\subsection{Delineating Land From Water in Intertidal Areas of Aerial Photographic Imagery}

Dataset C (Figure 4F, Table 1) consists of a series of 10, three-band arbitrary images of shoreline environments such as could be collected from a low-altitude aircraft in numerous locations, each labeled by five people using the following four classes; 1) deep water, 2) whitewater, 3) intertidal area (including all visibly shallow water where the surface below the water is visible, swash regions, and wet sand), and 4) dry land. The reader is referred to Figure S7 that depicts all ten images. Such imagery is useful for basic monitoring and photogrammetric reconstruction of shoreline environments.

Five labelers examined the same complex imagery that is readily interpretable without specialist knowledge but like dataset B, is not necessarily straightforward to consistently interpret. It is a complex labeling task involving identification and lumping of intertidal areas of what are in fact two distinct classes, namely wet sand and shallow water, into a single 'shallow' class. The task is made even more complex by asking the labelers to distinguish between that shallow class and 'water', a subjective choice requiring identification of water that is deep enough so as not to be confused with shallow water through which the underlying surface is visible. On this occasion, the labeling team of five people discussed the challenges of reliably distinguishing among these four classes beforehand, and this labeling exercise was to determine the utility of the class set before a larger labeling exercise was conducted. 
Doodler: Human-in-the-Loop Segmentation of Earth Surface Imagery

\subsection{Benthic Physical Habitat Mapping in Sidescan Sonar Data}

Dataset D (Figure $4 \mathrm{~d}$, Table 1 ) consists of a non-continuous spatial series of 51, one-band (greyscale) image tiles, each a short section of port or starboard scan consisting of 1024 consecutive sonar pings stacked as image columns. The length of each ping varied due to sonar range, resulting in the number of image rows varying between 1300 and 2000 pixels. The scans are collected using a Humminbird Solix sidescan sonar emitting a frequency modulated sound pulse with a nominal carrier frequency of 1.2 MHz, from sections of the Pearl River and its tributary the Bogue Chitto, and from the Chickasawhay, Buoy and Leaf tributaries of the Pascagoula River, in Spring 2021, for mapping in-stream physical habitats in coastal plain rivers of Louisiana and Mississippi. Dataset D (Table 17) consists of 10 example scans from the Bogue Chitto River, four from the Buoy River, two from the Chickasawhay River, 12 from the Leaf River, and the remaining 23 from the main stem Pearl River. The samples are selected for a variety of substrate types, water depths, and turbidities. Data are decoded and processed following Buscombe [2017]. The reader is referred to Figure S8 for more example images.

The pixels represent acoustic backscatter intensity (brighter $=$ higher intensity) of the 80-ms pulse, mapped in a non-linear coordinate system representing two-way travel time on the y-axis, and pulse number on the $\mathrm{x}$-axis. Because the transducer moves, pulse number corresponds to along-track distance, but the scale varies with boat and current speed. The top portion of the y-axis records backscatter from the water column and represents a nearly vertical domain between the transducer and the river bed. The lower portion records backscatter from the river bed at increasing distances from nadir. As the distance increases (lower in the images), the sound-path angle of incidence increases, changing the distance scale. The pixels representing the water column are oriented perpendicular to the bed, and the remaining pixels representing the riverbed and shadows 
Doodler: Human-in-the-Loop Segmentation of Earth Surface Imagery

in the lee of the bed and other objects. The water column pixels are therefore $2 \mathrm{D}(\mathrm{x}, \mathrm{z})$ and the remaining pixels are 2D (x,y) representations of the 3D (x,y,z) bed relief; objects on the bed cast shadows in their lee, the length of which depends on the geometry of the object with respect to the sonar [Buscombe et al., 2016]. The length of each ping is variable, depending on the characteristics of the sound pulse that collectively determine range, and the fact that the amount of usable data also varies strongly across-track (the vertical image dimension) due to attenuation of sound by water and the bed [Buscombe, 2017].

Like many scientific images, there are unusable portions of the imagery that would need to be removed through classification and removal by an automated process; in this case, they are the bank shadows and water classes, because the others are mappable in 2D space. There are many low-signal-to-noise (dark, grainy) textures that at small scale are not distinguishable without some spatial context - such as water, and shadows cast by variously sized objects. The full class list is as follows: 1) water; 2) shadow/riverbank; 3) shadows cast by instream objects and morphologies; 4) submerged wood; 5) fine sediment bedforms; 6) flat, fine sediment; 7) coarse sediment (gravel through boulders), bedrock, and vegetation; 8) anthropogenic (human-made objects); and 9) unknown (rare blank regions where the sonar recording cut out). Of the above, all but 'anthropogenic' are present in the dataset used for this study.

Such imagery is used to compare the products resulting from two labelers annotating the same complex imagery requiring specialist interpretation. Such imagery is used for mapping riverbed sediments [Buscombe et al., 2016, Buscombe, 2017] to provide basic information for benthic habitat mapping, and morphodynamic and sediment transport studies in rivers. It is also an example of a geophysical dataset with features in common with other Earth surface imagery, such as slices from 3D tomography data, Synthetic Aperture Radar (SAR), multibeam sonar backscatter, seismic reflection and refraction, to name but a few. The sidescan dataset requires the most training and 
Doodler: Human-in-the-Loop Segmentation of Earth Surface Imagery

expertise to interpret. It is the only dataset used here that is actively sensed (using an emitted sound wave and recording the echo).

Other than the false-color satellite imagery, this sidescan imagery is the only dataset that requires specialist knowledge to even sensibly interpret. Those data are therefore labeled by two experts with extensive prior experience in visual/manual interpretation of fluvial morphosedimentary forms. The other datasets (aerial and orthomosaic imagery) are passively sensed (photographic) and readily interpretable in the visible color spectrum (Table 1), requiring no special training however, that does not necessarily mean the labeling task is less difficult.

\subsection{Coastal Lagoon and Barrier Beach Dynamics in False-Color Satellite} Imagery

Dataset E (Figure 4e, Table 1) consists of a time-series of 40, three-band false-color 10-m (122 x 342 x 3 pixels) Sentinel-2 satellite images of coastal lagoon environments in Salinas Rivermouth Natural Preserve and National Wildlife Refuge in Monterey, California, collected between 31 December 2018 and 19 May 2021. The false color images consist of near infrared (band eight), red (band four), and green (band three). This three-band combination is commonly used for visual landscape classification where vegetation is present [Vuolo et al., 2016] because plant-covered land appears deep red, and denser plant growth is darker red. Water appears blue/black. The spatio-temporal time-series depicts various changes on the landscape, including the dynamics of the Salinas River mouth into the coastal ocean, surfzone and riverplume characteristics, changes to marsh and dune vegetation, and agricultural crop rotation. Therefore we defined the following classes: 1) water, 2) whitewater, 3) bare sand, 4) marsh veg, 5) dune veg, 6) crop/woody, 7) soil. The reader is referred to Figure S9 for more example imagery.

This imagery is further used to study the dynamics of beach breaching by a coastal river, 
Doodler: Human-in-the-Loop Segmentation of Earth Surface Imagery

and to compare the variability in geomorphic interpretation resulting from automated analysis of labels from three labelers labeling the same relatively complex imagery. Such imagery could be useful for opportunistic monitoring of coastal change from, among many potential uses, shoreline detection and characterization to assess trends in erosion and deposition, to assessments of habitat loss, flooding, surf zone hydrodynamics, agricultural development, bluff and sand dune dynamics. The frequency of important change at the coast is often greater than the frequency of available aerial platforms to provide imagery, especially in remote locations at short notice, and this makes the vertical and time-varying components of these landscapes especially difficult to unravel from opportunistic surveying/sampling. Satellite imagery with its regular timestamp therefore has a crucial role to play in linking time and spatial scales at coasts [McCarthy et al. 2017], and will play an increasingly important role in facilitating coastal science as imagery becomes higher resolution and better quality, and new sensors provide capabilities to sense new quantities [Vos et al., 2020].

\subsection{Coastal Evolution in Satellite Imagery}

Dataset F (Figure 4f, Table 11) consists of a time-series of 43, three-band visible-band pan-sharpened 15-m Landsat-8 satellite images (768 x 768 x 3 pixels) of Cape Hatteras, Cape Hatteras National Seashore, North Carolina, collected between 15 February 2015 and 27 September 2021. Dataset F differs from dataset $\mathrm{E}$ in three important respects; a) imagery represent a larger area of over 10 kilometers in each horizontal dimension; b) imagery is visible-band; and c) the dynamics captured, consisting of changing sandbars, sandwaves, beaches and wave breaking patterns, manifest over a larger timescale ( 79 months compared to 18 months of dataset E). We labeled the following classes: 1) water, 2) whitewater (surf), 3) sand, 4) land (all dry land that is not sand). There are also some small clouds and shadows of clouds in the scene, all occurring above water, therefore they are labeled 'water'. However, seperate classes for clouds and shadows might also be a valid strategy. 
Doodler: Human-in-the-Loop Segmentation of Earth Surface Imagery

The reader is referred to Figure S10 for more example imagery. This larger-scale (multi-km) imagery is used to demonstrate the utility in segmenting natural features at relatively large scales, and is also used to compare hand-digitization workflows with the methodology presented here.

\section{Case Study Results}

\subsection{Image Size and Resolution}

A comparison of label images at the two different grid sizes helps us understand at what grid size, and perhaps more importantly image size, we should ideally use for a given scene. A region of the 5-cm and 25-m pixel imagery in dataset A [Sherwood et al., 2021] are divided into $1024 \mathrm{x}$ 1024 x 3 pixel tiles for annotation, which resulted in 99 and six tiles for the respective resolutions. It is more difficult to accurately label the larger, coarser resolution imagery for two reasons: the $25-\mathrm{cm}$ imagery covers a much greater spatial extent than the 5-cm imagery, so features are smaller, and the imagery is less well resolved, therefore features are less distinct. However, images can be over-resolved for the task, and the time it takes to label a set scales approximately proportionally, at best, with the number of images in the set.

Each of the image tiles are labeled, then merged back into large label orthomosaics on the same spatial grids as the original orthomosaic images (Figure 5). In this case, errors are more readily observed when image tiles are merged, and assessed visually. We found this for both the 25-cm imagery and the 5-cm imagery; in Figure 5, those regions appear as abrupt changes in label values and are indicated by white boxes in Figure 5 e through $\mathrm{h}$. This artifact is more common for the coarser-resolution $25-\mathrm{cm}$ imagery. The purpose of tiling of large imagery is to make the labeling tasks more manageable, and it also typically makes labeling faster. The disadvantage is that many of the errors in the higher resolution imagery occur or become apparent at tile boundaries. These 

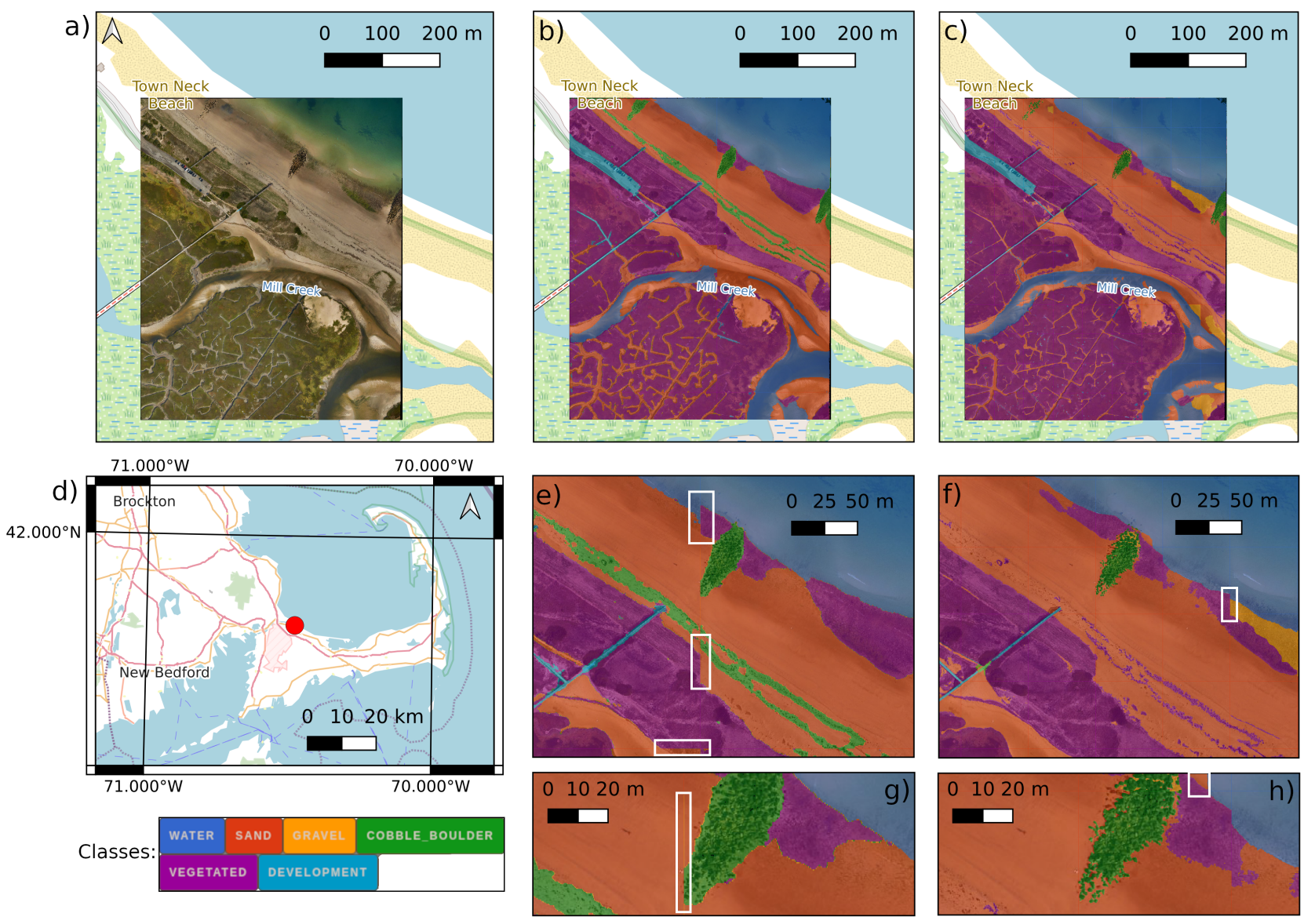

Figure 5: a) A region of orthomosaic of Sandwich Town Beach (dataset A); b) 25-cm label imagery as a semi-transparent color overlay; c) 5-cm label imagery as a semi-transparent color overlay; d) geographic location of the site; e) closer detail of b); f) closer detail of c); g) yet closer detail of e); and h) yet closer detail of f). In b), c), e) and f), label imagery consists of small 1024x1024 pixel label tiles that have been combined into a raster of full extent in a GIS. Classes are also depicts as colorful buttons (the same buttons used in the program Doodler when used to make the label tiles). White boxes highlight regions discussed in the text. 
errors are generally either caused by a) a relatively low spatial density of annotations compared to the higher-resolution imagery, or b) by annotations omitted by the labeler due to the larger size of the imagery. The majority of such errors occur at label boundaries and could be ameliorated through use of a spatial low-pass filter.

Other errors are due to misidentifications due to the lower resolution of the imagery; note how in Figure 5e the wrack line is labeled green (cobble/boulder), whereas in Figure 5f it is labeled 'vegetated.' The latter is perhaps more correct, because it is composed of dead vegetation. The task became ambiguous, because wrack is rough like cobbles but composed of organic matter. In addition, the wrack is much better resolved and identifiable in the 5-cm imagery. For this class set, we would use moderately low resolution imagery for this segmentation task, but small image tiles. However, the decision is dependent on the processes of interest. In this example, spatially less extensive, higher-resolution image tiles would be useful for delineating subtle differences in sedimentary grade or texture that only manifests at that scale, such as the difference between fine and coarse sand. Coarser resolution imagery may be sufficient for delineating the more obvious sedimentary transitions, such as gravel to boulders. Before embarking on segmentation tasks where image grid size can be varied it is recommended to use an exercise similar to this to determine a grid resolution and image size that is a good compromise for available time, required spatial density of annotations, and ideal image size where the smallest important features are visible (e.g., higher resolution may be needed for identifying animals or distinguishing between subtle sediment or vegetation types).

\subsection{Inter-Labeler Differences}

Dataset B is used to compare the products resulting from two labelers labeling the same complex dataset. The mean agreement is high (Figure 6), as evidenced by a median of mean Dice scores of 
Doodler: Human-in-the-Loop Segmentation of Earth Surface Imagery

0.76 , and Dice scores are generally only marginally higher than equivalent IOU scores, suggesting class imbalance is not too much of a factor for this dataset. There are many more examples of where Dice $>>$ IOU (i.e., IOU-Dice residual in Figure 6r is greater than, for example, 0.075), than where Dice and IOU are close.

\subsection{Class Selection}

An analysis of the labels generated from dataset $\mathrm{C}$ presents an opportunity to discuss labeler agreement when a classification task is somewhat subjective, and how to achieve consensus by identifying which classes to lump together, and which to keep separate. IOU and Dice scores are surprisingly good (Dices scores range from 0.87 to 0.93 ) when evaluated over the full set of 4 classes (Figure $7 \mathrm{a}$ ) and show greatest improvement (Dice scores range from 0.94 to 0.97 ) when the whitewater class is included with the deep water class and shallow is lumped with the dry land class, to create a binary or two-class set (Figure $7 \mathrm{p}$ ). Any remaining low scores are partially the result of confusion over whether to include swash foam as whitewater. All Dice and IOU scores increased when evaluated over two classes instead of four, although not uniformly (Figure 7), suggesting class imbalance is variable. Analysis of a set of labels in this way from multiple labelers could also be used to identify any outlier labelers whose interpretations are different from the rest of the group. As in evident in Figure 7, there are no individuals among the five labelers who have a noticeably lower agreement.

\subsection{Specialized Labeling}

Dataset $\mathrm{D}$ used to compare the products resulting from two labelers labeling the same complex imagery requiring specialist interpretation. In this case, the mean agreement is lower than for the NOAA aerial imagery, as evidenced by a comparitively low median of mean Dice score of 0.43 

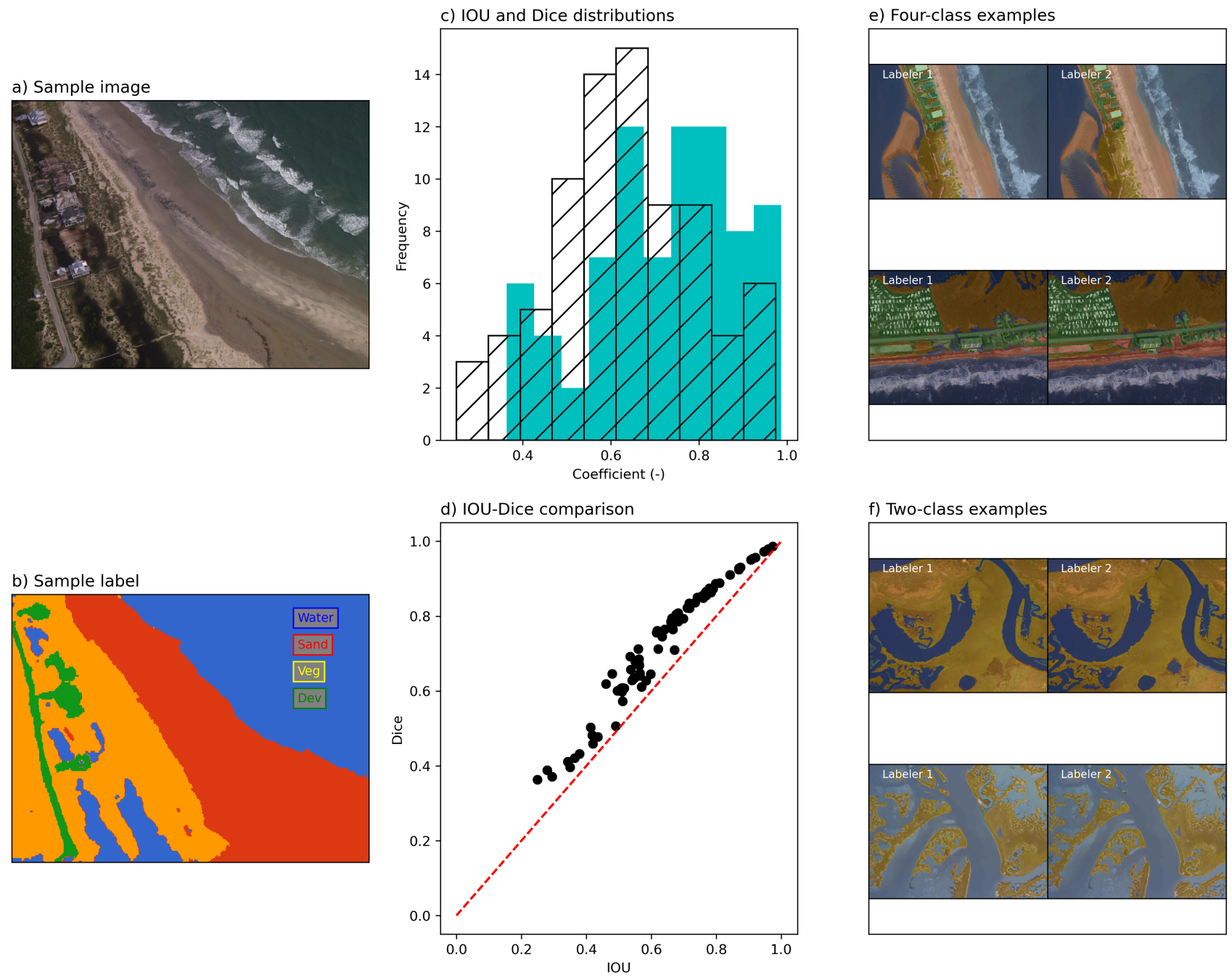

Figure 6: a) Sample image from the dataset; b) Label image associated with b); c) Histograms of Intersection over Union (IOU) and Dice scores for the 80 pairs of labeled aerial images; d) IOU-Dice comparison; e) Examples where mean Dice $>0.075$ than mean IOU; f) Examples where mean Dice and mean IOU are within 0.075 . 

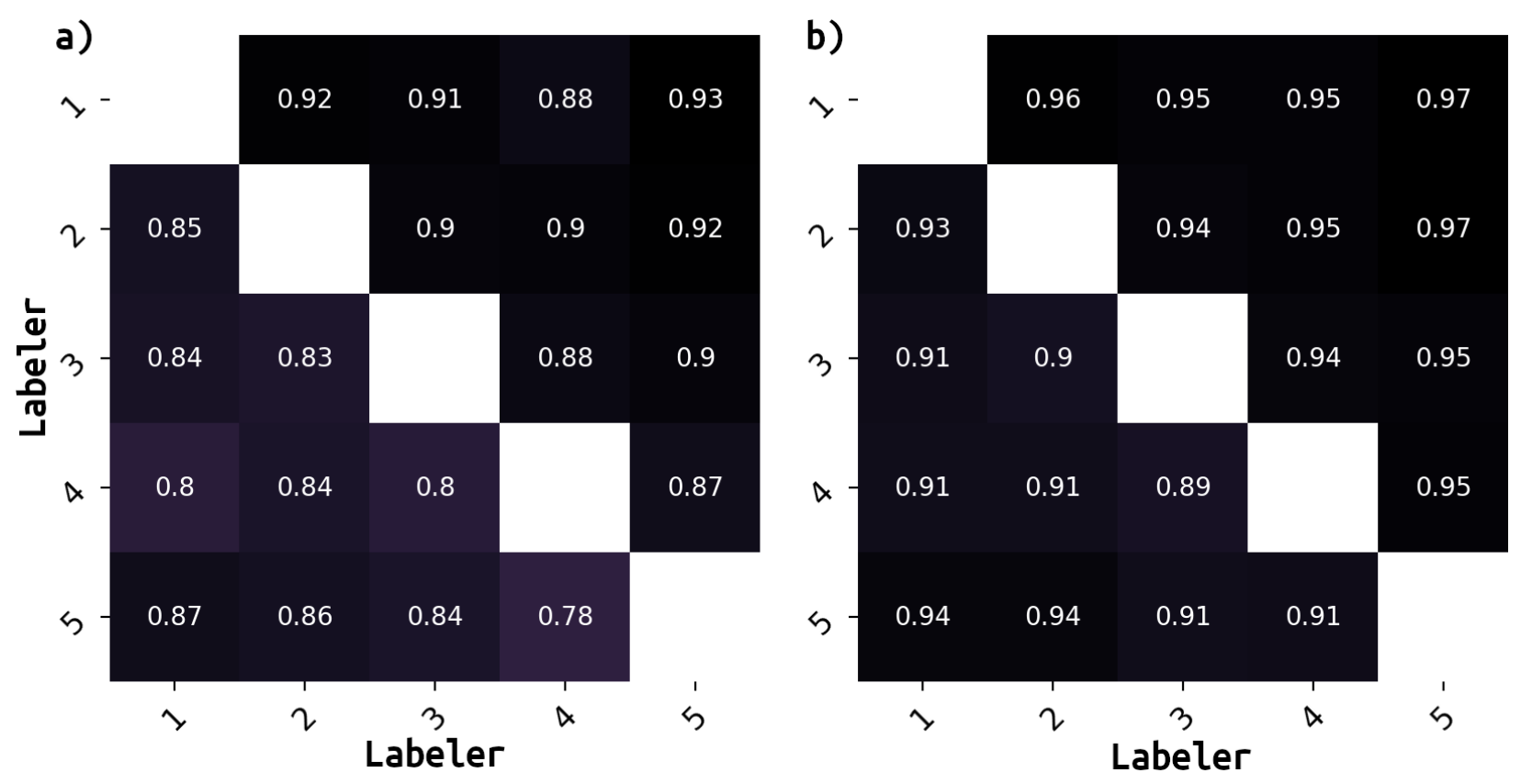

Figure 7: Matrices quantifying agreement among five labelers numbered one through five. The upper-right half of each matrix shows Dice scores, and the lower-left have shows Intersection over Union (IOU) scores. Two labelling experiments are shown: left (a) used four classes (deep, white, shallow, and dry); right (b) used two classes, combining 'deep' and 'whitewater' as one class, and 'shallow' and 'dry' as the other. 
compared to 0.76 for the NOAA aerial imagery (compare Figure $8 \mathrm{a}$ and $6 \mathrm{a}$ ). This is possibly due to the task being more difficult, meaning large areas can be legitimately called two different classes (examples are shown in Figure $8 \mathrm{~d}$ and e), and because there are more classes (eight instead of four), meaning the class-averaged IOU or Dice is affected by outlier classes.

Another major reason for the generally lower scores is that having more classes presents greater opportunity for a mismatch in the number of respective classes in each of a pair of label images. Recall that where different numbers of unique classes exist, i.e., two different candidates for $k$, we choose $k$ as the maximum length of the two respective class sets. The sidescan label set has, among those used in the present study, a greater percentage of images like this where there are unequal numbers of labels per image, therefore a greater percentage of conservative scores, which further decreases the class-averaged score.

Set-averaged Dice and IOU scores (i.e., the scalar mean of a distribution of mean scores) are close (Figure 8 8 ), suggesting any class imbalance is not affecting the comparison between labels. Class imbalance may not be avoidable if specific classes must be used for the scientific purpose the labeled imagery serves, however the effects of class imbalance can be reduced by merging appropriate classes, i.e., a minority class into a majority class, where possible. If a class is infrequent, but deemed too important to miss, imagery could be cropped so the class imbalance issue is ameliorated, or the algorithms could be modified to use class weights.

The two examples shown in Figure 8 8 e with relatively poor agreement do so for different reasons; in the upper example the two labelers have disagreed over the two shadow classes, and in the lower example the two labelers have disagreed where one identifies a region as coarse whereas the other identifies it as wood. In these examples, consensus could be achieved through some rules-based process, or by redoing the labels with lower-than-average IOU and/or Dice scores in order to achieve greater label precision through consensus [Monarch, 2021, Goldstein et al., 2021]. 
a) Sample image

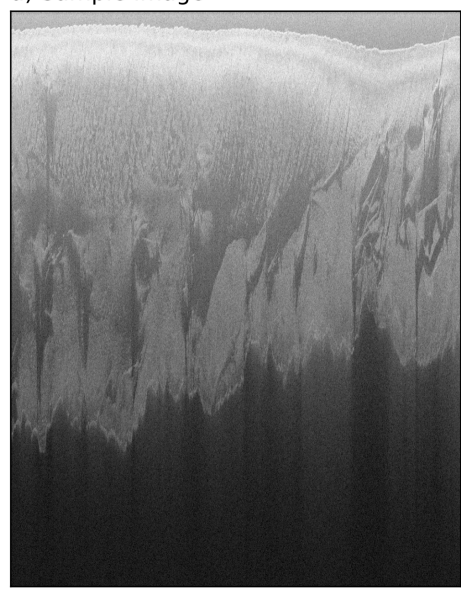

b) Sample label

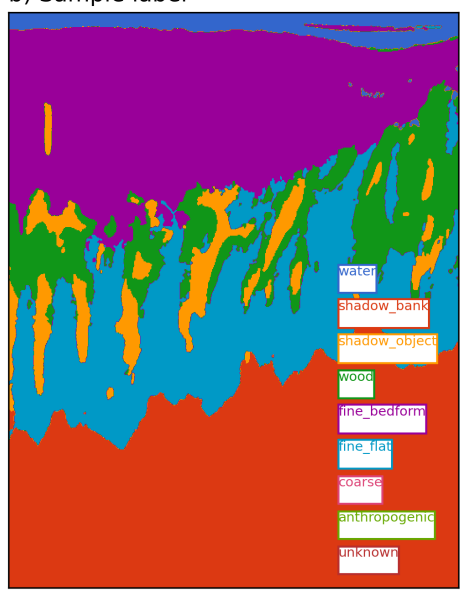

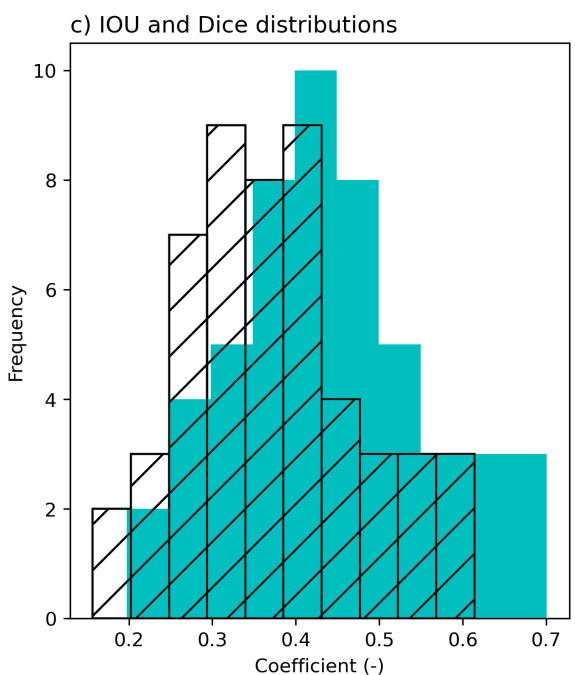

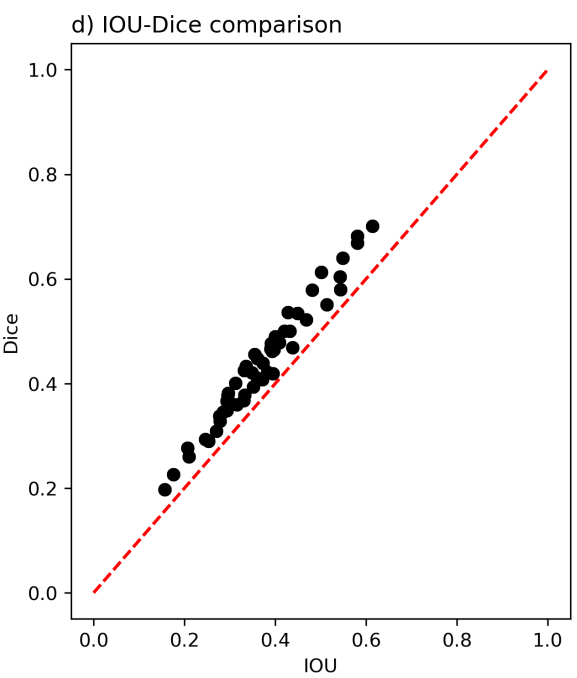

e) Average/good agreement

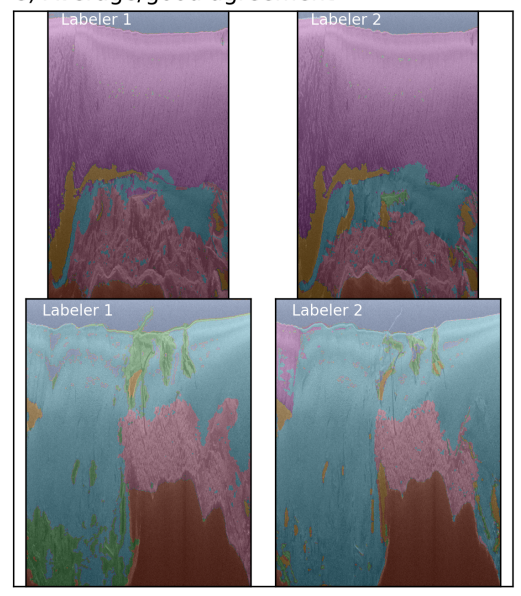

f) Relatively bad agreement

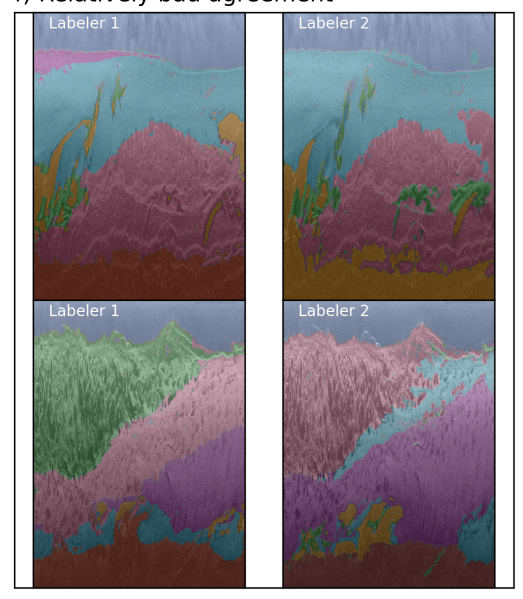

Figure 8: a) Sample image from the dataset; b) Label image associated with b); c) Histograms of mean (class-averaged) Intersection over Union (IOU) and Dice scores for the 51 pairs of labeled sidescan images; d) sample mean IOU -mean Dice comparison; e) two examples of average/good agreement; and f) two examples of relatively bad agreement. 
Doodler: Human-in-the-Loop Segmentation of Earth Surface Imagery

630

\subsection{Multi-Labeler Comparison of Quantifying a Geomorphic Process}

Dataset $\mathrm{E}$ is used to compare the products resulting from three labelers labeling the same complex imagery of a geomorphic process. The overall agreement between Labelers 2 and 3 is very high, as evidenced by a mean Dice of 0.9 (Figure 92). Additionally, the distribution of scores between Labeler 1 and Labelers 2 and 3 are almost identical.

In this case, mean Dice scores always exceed mean IOU scores (Figure 9b,c), suggesting class imbalance does affect the comparison between labels (water is by far the dominant class in every image). The two largest discrepancies between mean Dice and IOU scores are shown in Figure 9d; in each case, the white arrow highlights the major error, which in both cases is the mislabeling of water, which, as the dominant class, has a disproportionately negative affect on mean IOU compared to mean Dice. A comparison between IOU and Dice can also be used to detect outliers. The highlighted outlier in Figure 9e corresponds to the pair of labels shown in Figure 9f, in which the one from labeler 3 is missing one category, whitewater, which the program has called sand and which would have to be relabeled.

As for the geomorphic event we wished to describe using the segmentation data, namely the barrier breaching and "resealing" event that happened between 25 January 2019 and 10 April 2019, captured by seven cloud-free images, Figure 10 depicts the breach vicinity in each of the seven images, with the contoured outline of the sand category of the image segmentation created by each of the three labelers overlain. In all but one case, shown by the white rectangle in Figure 10g, all three labelers captured the outline of the barrier correctly, in the vicinity of the breach, plus the back barrier and shoreline areas. There are two additional images showing more temporary breaching events (on 24 April 2020 and 28 February 2021) in which all three labelers captured the outline of the barrier correctly (not shown). The average horizontal variability between outlines for the three 
a) Sample image

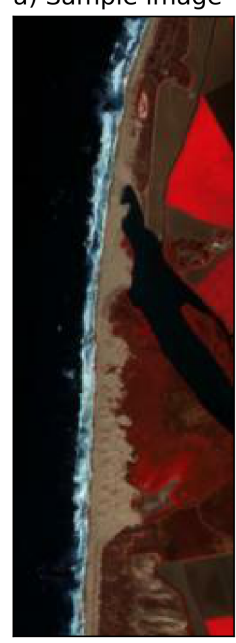

b) Sample label

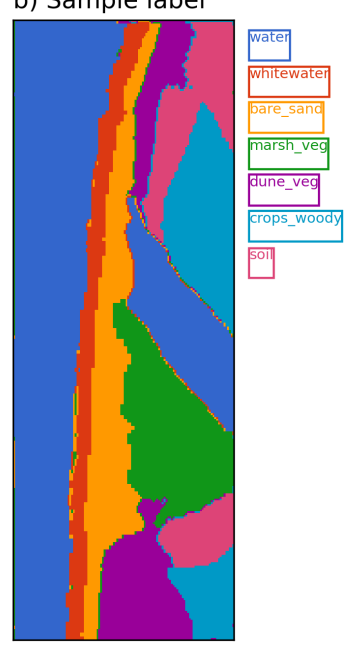

c) IOU - Dice residual

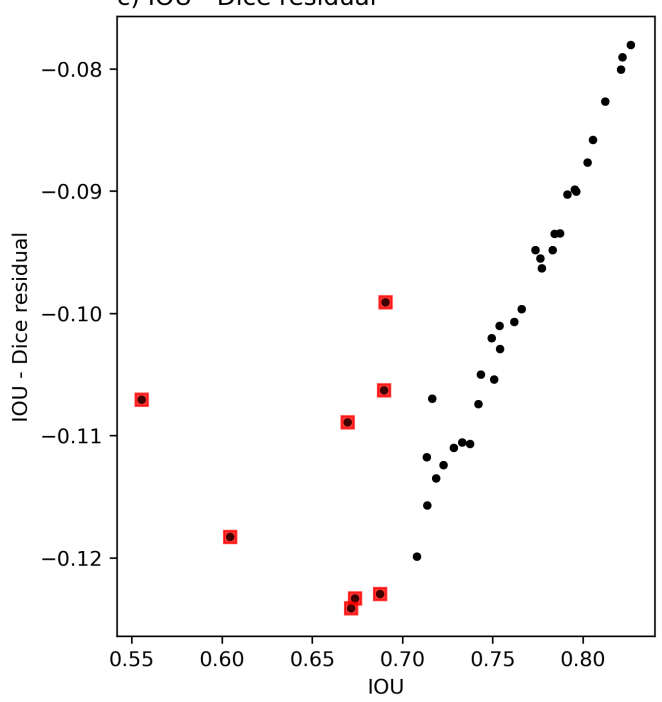

d) Agreement matrix

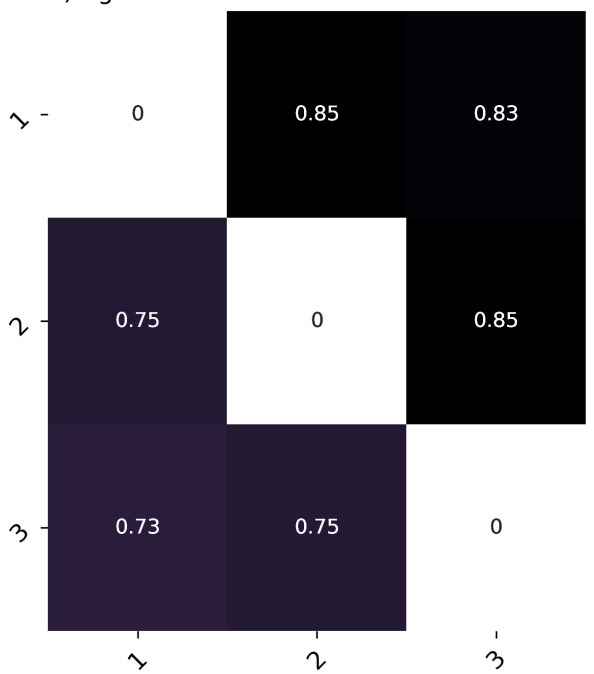

e) Average/good examples

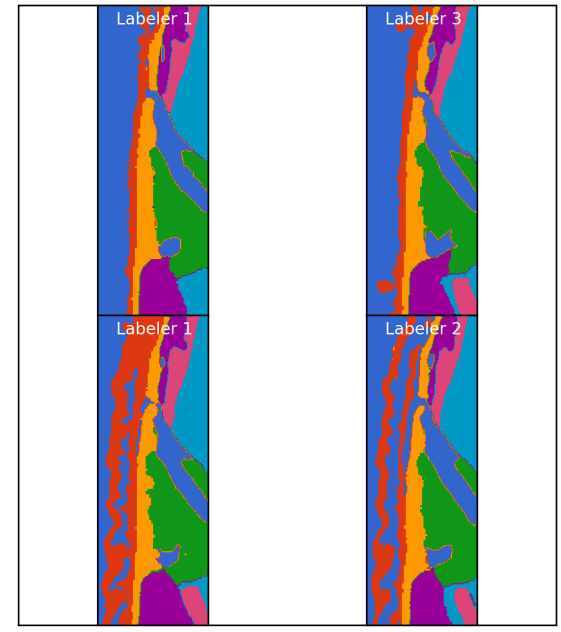

f) Relatively poor agreement

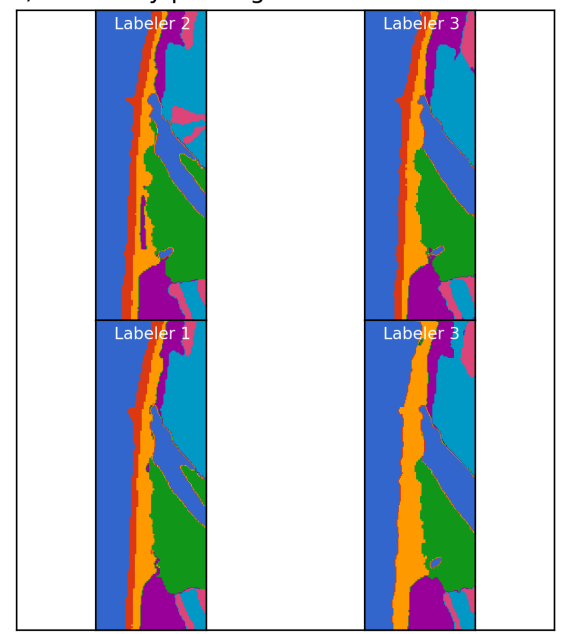

Figure 9: a) Sample image from the dataset; b) Label image associated with b); c) mean IOU versus mean IOU-mean Dice residual for the 80 pairs of labeled multispectral satellite images, highlighting outlier labels; d) IOU (bottom left matrix elements) and Dice (top right matrix elements) scores among all 3 labelers; e) two examples of average/good agreement; and f) two examples of relatively bad agreement. 
Doodler: Human-in-the-Loop Segmentation of Earth Surface Imagery

respective labelers is within two pixels (20-m horizontal ground distance).

Aside from specific cases like those described above, a potential more generic downside of using highly discriminative models optimized for specific tasks is that they do not necessarily transfer well to out-of-distribution data. This is why Doodler works well to generate training data for other types of models that carry out segmentation on datasets at scale (i.e., with much more variety than a single image). To demonstrate how the MLP model framework does not transfer well to unseen data, and hence why for fully automated segmentation of unseen sample imagery requires a more powerful approach such as a deep neural network trained on thousands of examples, we use dataset $\mathrm{E}$ once again. For each of the 40 images, we used the MLP model built on the small annotated scene to apply to a scene with an extent twice as large, extending down coast. The MLP model trained on each half image is able to extrapolate the broad categories that are significant at the boundary of the extent of annotations well, i.e., at the bottom edge of the top half of the image (Figure 11) such as water, dune, and crops. However, it tends to under-predict the less dominant classes whitewater (surf), soil and sand, and predictions get worse the farther away from the boundary. The CRF model cannot fix all the errors in these under-predicted classes however, the Doodler program itself results in annotations that could be used within alternative ML frameworks and it is likely that annotations with sufficient density for a good MLP solution would easily be sufficient for a more sophisticated model (perhaps at greater computational expense) because MLPs are relatively simple ML architectures. The fact that the annotations have been optimized through guided iteration towards a solution for a particular ML algorithm, does not mean they cannot be repurposed for, after all, they are simply example pixels of each class. And, as we mentioned above, Doodler is designed for both one-time dataset segmentation and for generation of label imagery for training ML models such as deep learning models for fully automated image feature-extraction and class segmentation at scale, for application to Earth surface imagery. 

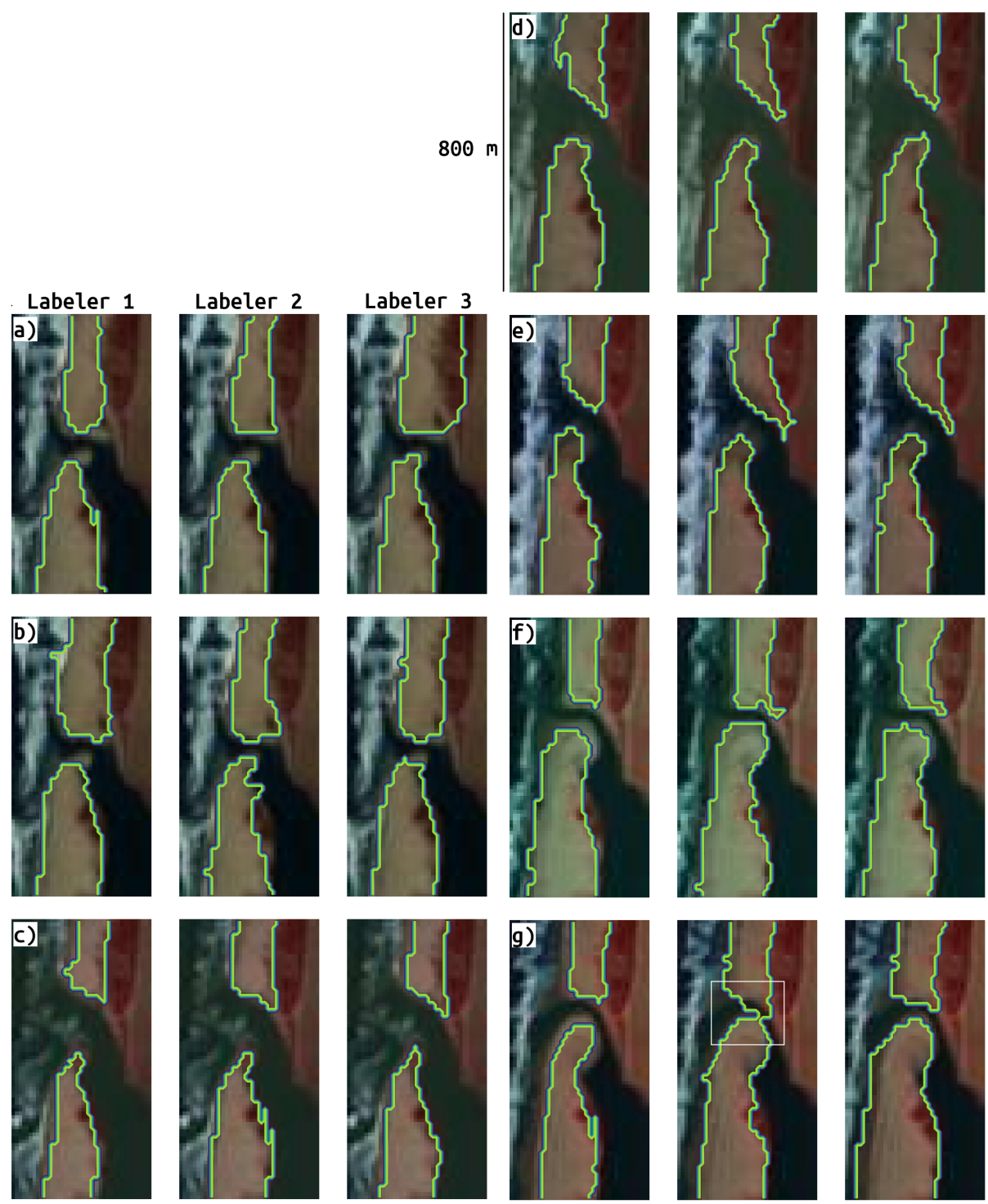

Figure 10: Subplots a) through g) depict the breach vicinity in seven images captured between 25 January 2019 and 10 April 2019, with the contoured outline of the sand category of the image segmentation created by each of the three labelers overlain. The white rectangle in g) shows the only case where the sand polygon would suggest the barrier is still sealed, albeit by a single connecting pixel. Otherwise, the agreement is very close, within two pixels typically with a maximum discrepancy of four. 

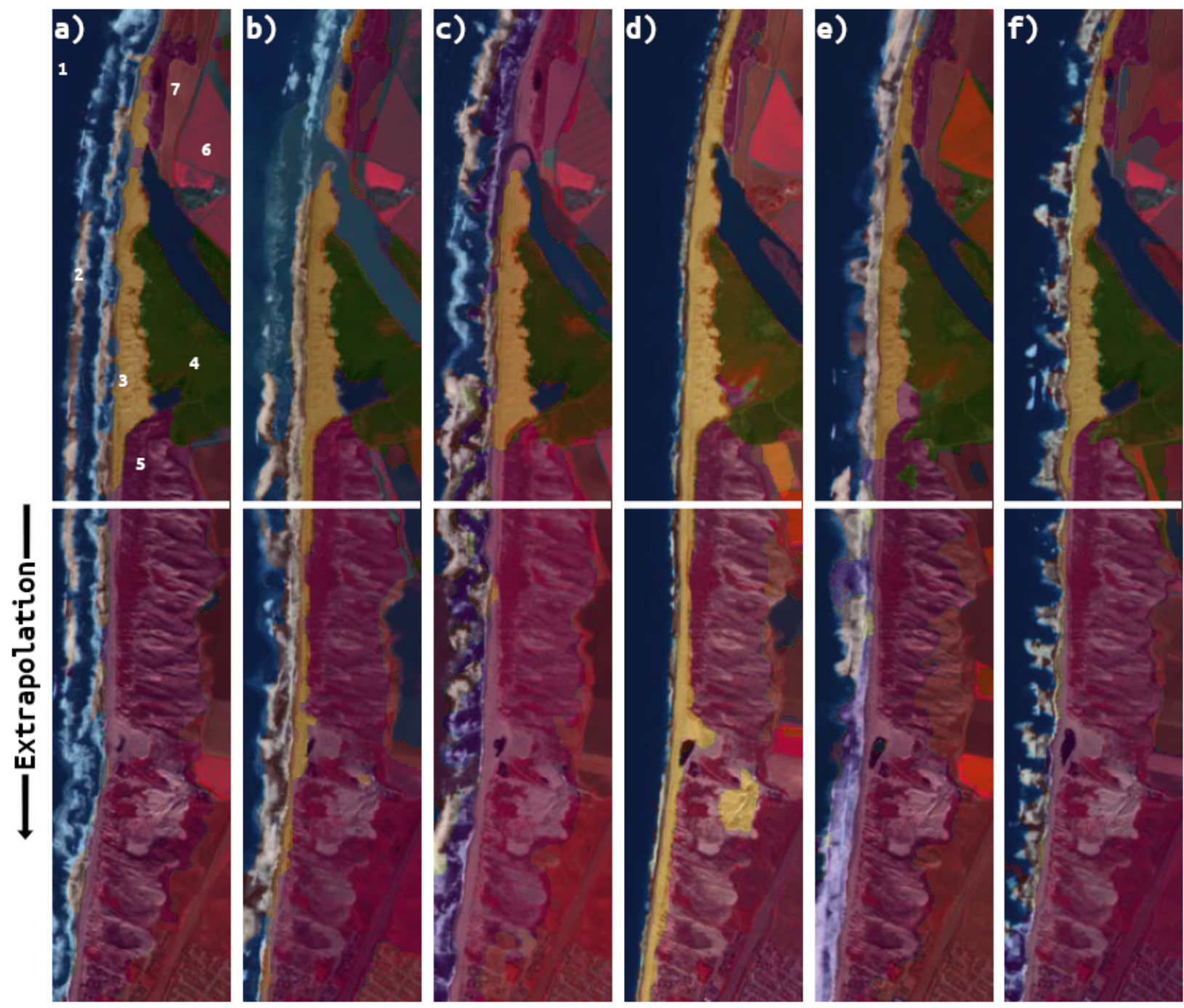

Figure 11: Output label images from a MLP model built on the small annotated scene above the white horizontal white line in the center of the scene, then applied to the entire scene with an extent twice as large. In the extrapolated region, water, dunes, and crops are reasonably well predicted, but sand, whitewater (surf), and soil are not as well predicted. 
Doodler: Human-in-the-Loop Segmentation of Earth Surface Imagery

\section{7 \\ 4.6 Comparison with manual digitization}

A single scene collected in 15 February 2015 (the first image in the collection) was annotated in a traditional way using hand digitization of polygons, then again using Doodler. This was conducted by the same individual on the same day. It took 7.5 minutes to carefully label the scene and compute the segmentation using Doodler. We used an open-source annotation software [Skalski, 2019] to efficiently hand-digitize polygons for the entire scene. This program has similar zoom and pan tools to Doodler, which enables careful labeling of small features such as the relatively narrow sand beach and the surf zone (multiple lines of breaking waves). Additional imagery showing the stages of digitization is provided as Figure S11. The manual digitization took 25 minutes, or more than three times as long. Whereas we could have conducted this comparison using any of the datasets presented in Table 1, we chose this dataset because the imagery is sufficiently large, and some classes sufficiently spatially limited, to warrant zooming and panning in order to accurately label. We note that the degree of zoom and pan is somewhat comparable between the two annotation programs, however the extent of annotation is much less with Doodler, and each annotation is much quicker to complete.

The digitized polygons were converted into a label image for direct comparison with the label image obtained using Doodler. A comparison of the inputs and results is presented in Figure 12. The mean IoU and Dice scores that quantify the agreement between the two label images are 0.48 and 0.5 , respectively. This is low because the mean agreement for the two minority classes 'surf' and 'sand' are only approximately 0.015 , whereas the agreement over 'water' and 'land' are approximately 0.97 each. Owing to the large class imbalance in this scene, quantitative comparison is limited. Qualitatively, we observe that the two label images differ in three important ways. First, there are a few small gaps in the label image where the labeler did not ensure matchup (or overlap) 
between adjacent polygons. This is a common limitation of hand-digitization, and here manifests most significantly as gaps between sand polygons, as indicated in Figure $12 \mathrm{~d}$ by numeral i, and between the marsh and the beach, as indicated by numeral ii. Second, extremely small/thin objects are more difficult to hand digitize, resulting in the omission of the very thin sand bar, indicated by numeral iii in Figure $12 \mathrm{~d}$. The presence of this bar is marginally visisble but also indicated by the adjacent breaking waves. Doodler was able to capture this feature properly (Figure $12 \mathrm{~h}$, numeral iv) with a few annotated pixels in this region. Third, in complex regions of transition where adjacent classes are indistinct at the level of zoom at which the labeler has chosen to label, such as near shore where waves are breaking on the sand beach, hand annotation generally results in overly coarse digitization compared to Doodler. Doodler is able to predict at the pixel level, whereas it is overly time consuming for hand digitization of polygons at the same scale. However, there are also advantages to relatively coarse hand digization if it preserves actual boundaries better than a model prediction instance. An example is indicated by numerals $\mathrm{v}$ and vi in Figure $12 \mathrm{e}$ and i, respectively; hand digitization has labeled the ocean side of the beach better than Doodler, however Doodler has better labeled the pixel-level detail in the lagoon side of the beach.

\section{Discussion}

\subsection{Obtaining High Levels of Agreement}

The results suggest that given knowledgeable labelers, the Doodler program produces consistent label images (segmentations), even for complex scenes with numerous classes, indicating that multiple labelers can be used to label a dataset and the results will be consistent and cohesive. The majority of errors in the labels are not necessarily due to the model but are consistent among labelers. The datasets shown here (Table 1 ) are a few among numerous datasets we have already 
Doodler: Human-in-the-Loop Segmentation of Earth Surface Imagery
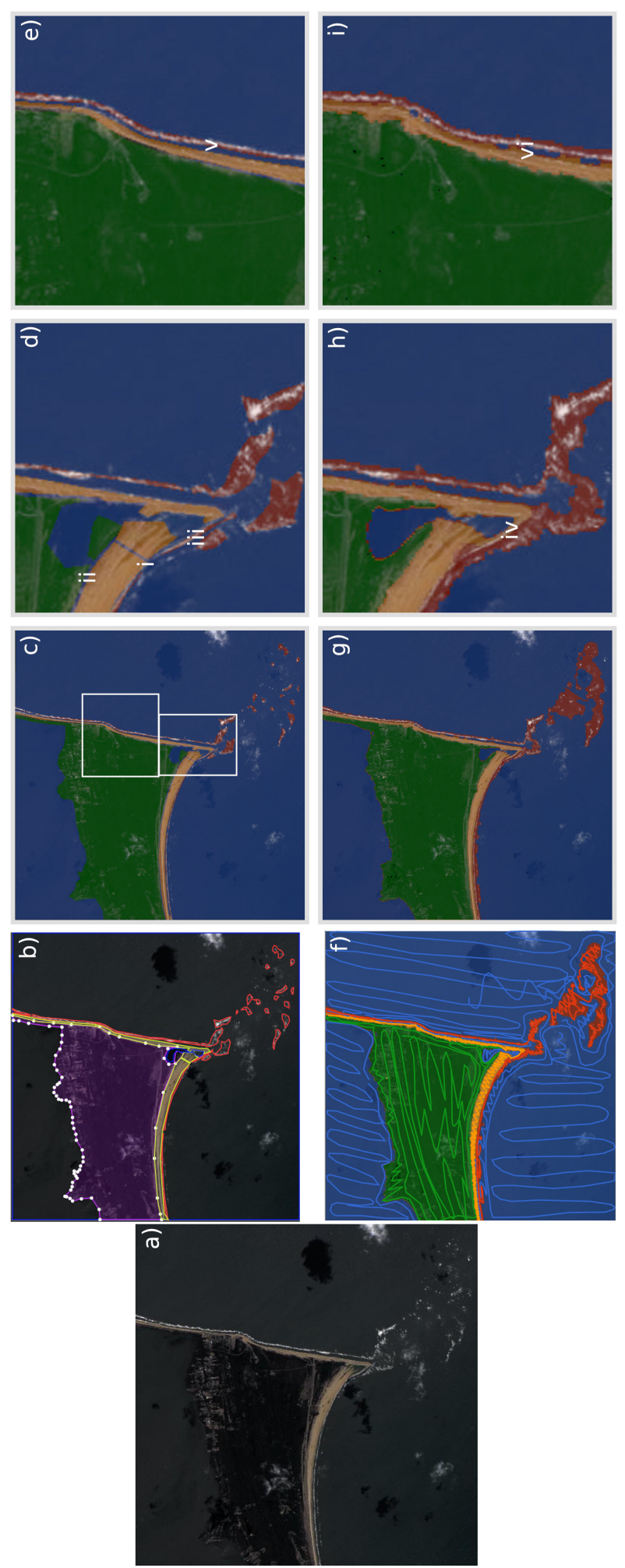

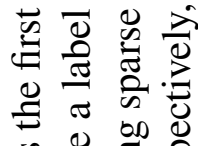

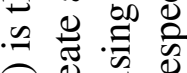

(త)

$\Phi$ 守

范

늘

进宓

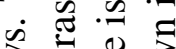

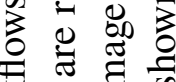

즘 $\cong$

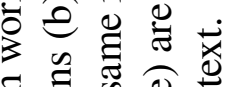

ธี อิ

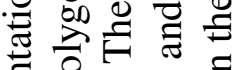

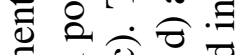

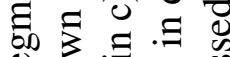

क Tं

웡

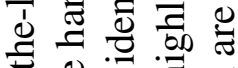

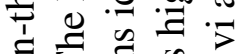

.

ฮี

灵穴边包

슬

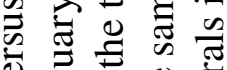

$>$ ప

ธี

乎的它它

:

- $\infty$ o

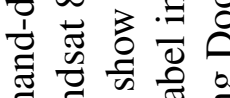

ปี

प्ञ $\cong$.

응

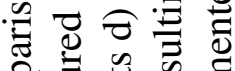

를웛

ठํㅠㅇㅠ

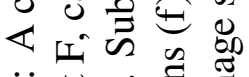

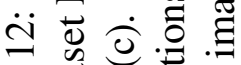

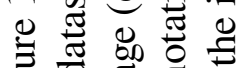

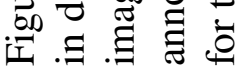


successfully used the program with, from millimeter-scale grid sizes in close-range photography to multi-decimeter-scale pixels in satellite imagery, using between two and many tens of classes. We also tried several previous software implementations for the basic idea, and have arrived at a user interface by testing hundreds to thousands of individual samples by dozens of individual labelers. By combining unary potentials from a discriminative MLP model that encodes the conditional likelihood of a class given an image feature, with pairwise potentials that encode the joint likelihood of image features and classes together, the CRF technique exploits the benefits of both discriminative and generative ML model frameworks, and almost always results in an as or more accurate image segmentation than using the discriminative MLP model alone as determined visually on thousands of label samples; the program can generate a side-by-side comparison of the MLP output and CRF output for any sample image.

An advantage of using a so-called 'cascade' of ML models whereby the outputs of the first is the inputs to the next (Figure 2), is that the second model can and often does revise the predictions of the first if they are inconsistent with the second. This situation can often arise because the confidence of discriminative ML models, such as MLPs, are as much a reflection of the model feature-extraction and classification processes (summarized by learned parameters, $\theta$ ) as the input data. That is why we say the model output is $P(y \mid \theta, \mathbf{x})$ rather than simply $P(y \mid \mathbf{x})$, to acknowledge the joint importance of model parameters $\theta$ with the specific image features $\mathbf{x}$ used during training.

Outputs are further improved by having a human in the loop, i.e., to immediately visually inspect segmentations for quality, and to add/remove annotations where necessary in places the model has mispredicted, and/or to adjust model hyperparameters (on an image-by-image basis if necessary). The percentage of imagery where such correction is necessary varies considerably by task (and to a certain degree the diligence of the individual labeler); on datasets tested to date, we estimate that approximately half or more of images require the addition of annotations beyond 
the initial sparse set, and approximately a tenth or less require the removal of annotations or the adjustment of hyperparameters. It is generally considered a good thing that the CRF solution is not overly sensitive to hyperparameter values, and that happens for several reasons by design (see section 2.4), because that allows the instructions given to labelers to focus on how to annotate well.

Based on comparitive exercises between hand-digitization using polygons and our alternative workflow, we conclude that our methodology encoded into the Doodler program is always faster; approximately 3 times faster for the imagery used in Figure 12, and up to 10 times faster for other imagery we tested that does not require as much (or any) zooming and panning. Faster labeling makes multiple labeler datasets easier to obtain, and multilabeler contexts have been shown to provide reliable label uncertainty metrics.

We also conclude that Doodler generally results in a segmentation that is as-or-more accurate than slower hand digitization workflows. First, Doodler ensures every pixel is labeled, whereas ensuring no gaps in the label raster that is the result of a hand-digitization workflow is difficult and often not managed. Additionally, Doodler picks up on pixel-level features that are too timeconsuming to label or invisible at a reasonable zoom level, especially in complicated regions of transition. As a result, labels are finer-scale and more accurate at the pixel level because errors at boundaries between classes that arise due to hand digitization, which can be significant because of mixed pixels or due to coarse digitization, are significantly reduced. Modeling the likelihood of uncertain regions is crucially important for class assignment in particularly difficult regions of imagery in a deterministic manner.

\subsection{Measuring Agreement}

In general, it may be qualitatively observed that any IOU score above 0.5 is a very high level of agreement at the whole-image level, especially for high-resolution imagery. One of the really useful 
aspects of both IOU and Dice as metrics is that they both penalize pixel-level noise, and scores are therefore an accurate reflection of high-frequency label noise, which tends to increase with higher resolution imagery. A comparison of aggregated IOU scores between pairs of labels in whole datasets also meaningfully reflects the difficulty of the task; sidescan scores are typically lower than aerial and satellite imagery due to relative difficulty in interpretation.

However, due to averaging over classes and uneven numbers of classes among samples and datasets, both IOU and Dice scores are best treated as comparatives within datasets. In fact, when evaluating agreement (uncertainty) on individual datasets, computing and comparing both Dice and IOU scores can be useful for various reasons. We have shown it is possible to use them to discuss ways to detect class imbalance, outlier labelers, and label images in multi-labeler contexts, as well as reporting mean agreement for multi-labeled datasets as an uncertainty and quality metric, among other potential uses. IOU is always the more conservative metric than Dice, and that can sometimes be useful when deciding on the subsequent uses of the data. While it is very sensitive to class imbalance, there are potentially a lot of advantages to measuring total error rate, the sum all different pixels (i.e., all false positives and false negatives) divided by the number of pixels in the image. The per-class IOU and/or Dice scores can show problematic classes where there is lack of agreement (Figure 13). For example, in the sidescan dataset (dataset D), the distribution of per-class scores has the largest range; shadow and wood classes achieve relatively little consensus (Figure 13p). The two shadow classes would likely have to be merged for consistency, and better agreement over wood and all the other categories might be possible if a manual documenting examples is prepared [Goldstein et al., 2021]. In the post-hurricane dataset (dataset B), sand is often difficult to distinguish from water for the same reasons as described for dataset C.. 

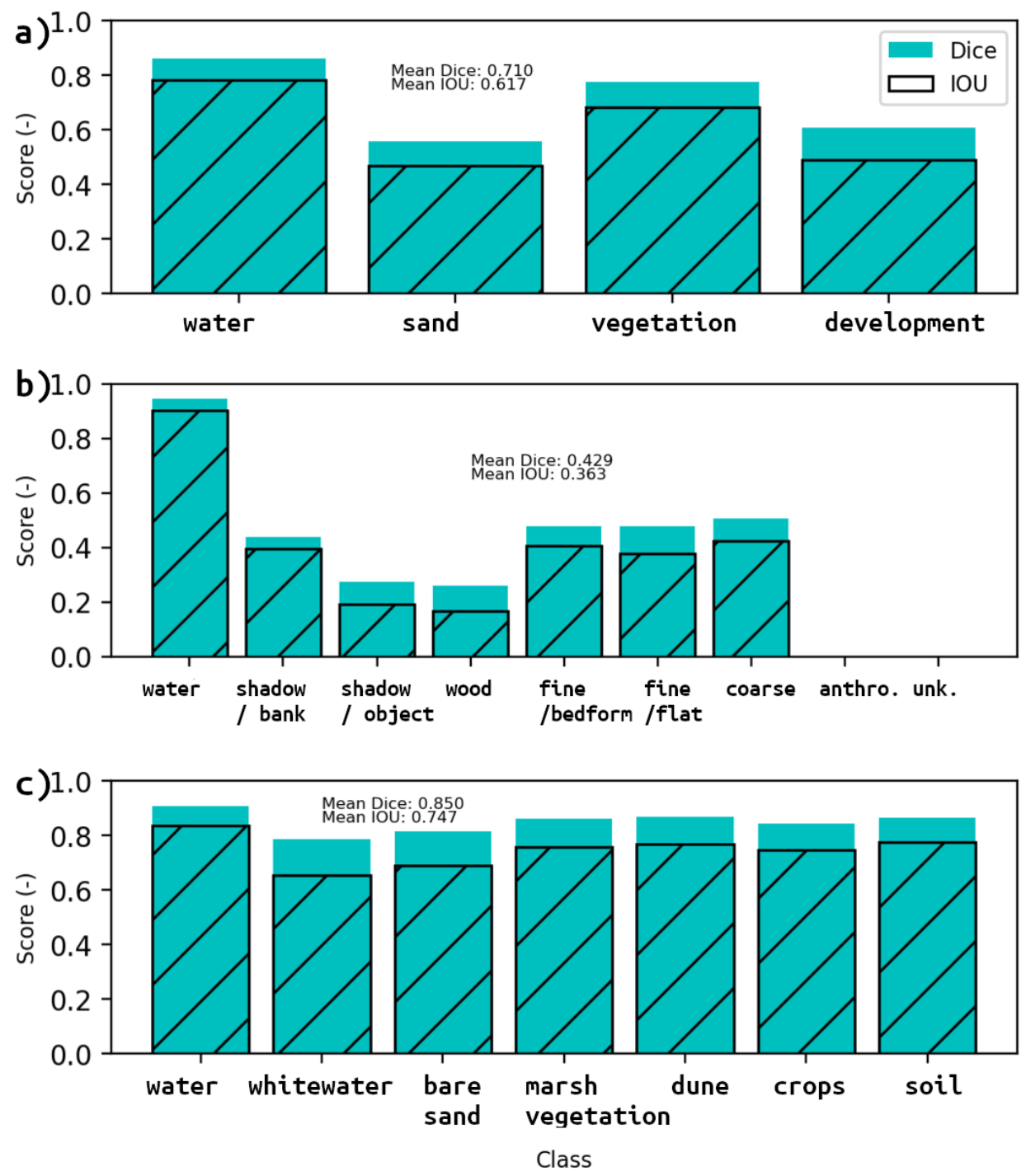

Figure 13: Per-class Dice and Intersection over Union (IOU; hatching) scores for a) post-hurricane aerial imagery, b) sidescan imagery, and c) satellite imagery 
Doodler: Human-in-the-Loop Segmentation of Earth Surface Imagery

791

792

\subsection{The Value of Sparse Annotations}

The sparse annotations provided by the human labeler are more valuable than the specific realisation of the fully labeled image. There are several reasons for this assertion. First, we tested alternative discriminative algorithms to the MLP that evaluate $P(y \mid \theta, x)$ on features $x$ that have already been extracted in a prescribed way. Among the alternative algorithms tested included the Random Forest and Support Vector Machine, both of which are used extensively in Earth surface processes research [Yao et al., 2008, Provost et al., 2017, Perry and Dickson, 2018] and worked well here too (see Figure S2 for representative comparison between MLP and Random Forest outputs). We chose the MLP because it is as or more accurate, with fewer model parameters, generally less overfitting, and had faster computation times. The key insight here is that the sparse annotations could be used with similar effect using a range of ML algorithms. This means that the Doodler program provides a means to acquire sparse labels that are optimal for a many ML frameworks to carry out segmentation, not just the specific ML framework (MLP and CRF) that we have presented.

Second, as labels, annotations are more valuable than the pixelwise label imagery because there may be better ML model frameworks to predict pixelwise class from the sparse annotations in the near future, but it may be much longer before computers are able to label complex Earth surface imagery unaided with human-level accuracy. In fact there may already be viable ways to use the sparse annotations directly to train deep learning models for image segmentation, for example by exploiting the variable spatial autocorrelation of each class [Hua et al., 2021] or by classifying image features as nearest neighbors in embedding space [Ke et al., 2021], however these techniques are currently much more computationally demanding, and would need large sparsely labeled datasets to achieve training convergence.

Third, the sparse annotations themselves encode the pixels chosen to represent the class in 
that region of the image, thus they are likely much better than a random selection of pixels from each scene and class at representing that class, perhaps efficiently encoding the line of greatest spatial transition (i.e., class boundaries). The CRF may on occasion (and by design) override the human label, and this may be quantified by locating (and/or counting) the pixels that differ in class between human input and CRF output. An analysis may reveal the degree to which and conditions under which the CRF over-rides the decisions of the human labeler. The Doodler program provides tools for extracting not only the sparse annotations and final projects, but also interim products, for any type of post facto analyses and evaluations.

Finally, the annotations themselves may be a proxy for other interesting properties of the data. For example, the spatial density of annotations may reveal areas of the scene that are more important for classification than others, or less ambiguous, or where the difficult transition areas are that the model is expected to predict. It is an interesting and as-yet under-explored supposition that there is some minimum sparsity of annotation necessary for a given target accuracy, but that would be complicated by the fact that multiple sets of annotations might give rise to identical outputs.

Other potentially informative derived attributes that relate to spatial autocorrelation and other spatial properties of the labeled regions include the spatial extent of each prediction, the shape the outline of that contiguously labeled region makes, and the spatial density with which annotations need to be made to properly segment the image. We find that the percentage of scene that is labeled for a satisfactory outcome varied with image size. It is between 10 and $20 \%$ for the sidescan imagery (1024 x 1300-2000 pixels) and between 10 and 30\% for the NOAA imagery (1000 x $750 \times 3$ pixels).

In both cases, there is no systematic tendency for one labeler to spend more time on labeling overall, although there can be significant differences over individual images. However for the $122 \mathrm{x}$ $342 \times 3$ pixel satellite imagery, the percentage is between 40 and $65 \%$. The percentages may be an 
overestimate of the labels actually necessary for a good image segmentation, because the default 'pen' (cursor) width is 3 pixels. That value is rarely changed by the majority of labelers in this study, although individual labelers tend to adopt that practice more readily than others, typically varying between 2 and 5 pixels depending on the scene. That is to say, it is possible that 1- or 2-pixel width annotations would have resulted in an equally good segmentation. That could be tested by using a morphological erosion operator on the sparse annotations then using the eroded doodles as inputs to the MLP and CRF estimation pipeline, and finally comparing outputs from full and thinned pen strokes. In some imagery used here, some labelers used thicker pens for the dominant classes, but others realized may have not done so because of the extra time it takes to change pen width. The number or spatial density of doodles, rather than thickness of pen, is generally a better local indication of scene complexity.

We found no significant correlation between either IOU or Dice score and percentage of the image annotated, either for individual images or for scores averaged over sets of labeled images. However, that is likely due to the fact that all labelers here are attentive and generally labeled a large percentage of the scene (between 10 and $65 \%$ of the scene, depending on image size) and in all areas of the image. Additionally labelers likely did so until the segmentation created from their sparse labels is satisfactory, i.e., it seemed to accurately represent the underlying scene. Annotations are somewhat different, and individual labelers were even sometimes identifiable by their unique style. However, in this study agreement among labels was not identifiably related to a labeler's individual labeling sytle.

The program outputs also provide the means to analyze the annotations (like quantify their spatial density) and compare them. It is generally a more effective and efficient strategy to add and remove annotations than use model hyperparameters to modify CRF model predictions, although of course both are sometimes necessary of the most difficult imagery. Other useful metrics to track 
Doodler: Human-in-the-Loop Segmentation of Earth Surface Imagery

include the total percentage of the image labeled, although in this study that is not correlated with any qualitative accuracy metric or quantitative agreement metric because all labelers were careful and attentive and not more detailed with one class than with another. However, total percentage labeled would reveal situations where a labeler consistently annotated too much or too little of the image, both of which can be a problem due to either model underfitting or overfitting the data.

\subsection{Future Work}

The most difficult imagery for Doodler would arguably be regarded as the most difficult for any image labeling program, namely degraded or poor quality imagery, and especially imagery where features and objects are small and hard to resolve because of low spatial resolution. Additionally, Doodler is not particularly well suited to labeling especially thin and short objects consisting of only tens to hundreds of pixels. For example, in large-format aerial imagery that represent large areas of ground, such hard-to-label objects would include individual pieces of driftwood, short and narrow paths and roads, vehicles, small buildings like cabins, people and other animals, among other common things. The common solution is to a) exhaustively label almost every occurrence of the small, thin classes, and b) to use a lot of zoom and panning, or smaller images, in which the labeler can better resolve the class and position the pen more accurately and precisely. However, because the CRF has agency it can override the human labels, and unfortunately tends to do so disproportionately for the more infrequent classes, which is almost always the classes associated with the small, thin objects. However, there are often trade-offs between available time and target accuracy with any labeling task. Therefore, on occasions when it is not efficient to use smaller images or spend time zooming and panning, especially if the main classification target is spatially extensive and/or continuous, the recommendation we would make is to classify the scene without employing the small, thin class(es); polygonal labels of those classes could be added later, rasterized, 
Doodler: Human-in-the-Loop Segmentation of Earth Surface Imagery

and merged with the label images of the other classes.

In section 5.3 we stated that annotations are more valuable than the pixelwise label imagery because there may already be viable ways to use the sparse annotations directly to train deep learning models for image segmentation. The recent semi-supervised method of Ke et al. [2021] is particularly representative of current trends in this scope, utilizing a concept known as contrastive learning [Wei and $\mathrm{Ji}, 2021]$ that learn the similarity between labeled and unlabeled data and base classifications on that similarity. The similarity is learned from the data, and the regions considered to be adjacent to each require some form of abstraction such as defining superpixels (contiguous segments of image based on location and color obtained by clustering algorithms) or perhaps another trainable model component. It is therefore a more complex solution. Whereas Doodler uses labeled pixels to assign classes to unlabeled pixels within each image, emerging ML techniques like $\mathrm{Ke}$ et al. [2021] also use those labels to assign classes within and across images. Such advances are possible by utilizing learned embedding representations of class-image pairings over larger datasets. Tools like Doodler would still be necessary to both collect the sparse annotations, and to generate independent data to evaluate the outputs of an automated technique for collections of images.

Although that was not carried out here in order to measure agreement over class sets and imagery among several labelers based on verbal instructions alone, upon inspection of the results we now recommend discussing and practicing candidate class sets with a small sample of imagery, and then having small a group of labelers trial, no matter how trivial the task may seem beforehand [Geiger et al. 2021]. Regardless of hypothesized degree of ambiguity in a given labeling task, individual labelers vary a little in terms of diligence and skill, and with a lot of Earth surface imagery there is an expectation for different labels in ambiguous regions of imagery, for the reasons discussed in section 1 . Therefore achieving consensus is a) part design, by using a modeling framework that is designed to objectively arrive at consensus in labels across the scene based on class-feature 
Doodler: Human-in-the-Loop Segmentation of Earth Surface Imagery

pixel pairings and b) part analysis, by analyzing agreement in segmentations of the same imagery by multiple labelers. Analysis of labels for the purposes of deciding on optimal class sets, and achieving consensus, is only possible when multiple labelers are used, although analysis of labels made by the same labeler on separate occasions might also have some value.

More sophisticated labeling workflows would include those that modeled the likelihood (confidence) of the sparse annotations themselves, or provided ways for the labelers themselves to provide that assessment [Monarch, 2021] and that may be the subject of future work. There is also much more work that needs to be done concurrently into strategies for selecting images to be labeled, such as active learning [Goldstein et al., 2020], automatically labeling data using embeddings [Ding et al. 2020] and other data representations that have been found by application learning and transfer-learning algorithms [Cunha et al., 2020], or discovered using synthetic data [Wu et al., 2019].

\subsection{Human-in-the-Loop Image Segmentation}

Scoping feasible applications of Deep Learning in the geosciences benefits from rapid prototyping of ideas, model frameworks, and trained models used in a transfer-learning workflows that are often inherited from other disciplines [Buscombe and Carini, 2019, Buscombe et al., 2020, Goldstein et al. 2020, Cunha et al., 2020, Yang et al., 2020]. The challenge is to evaluate their utility using domain-specific labeled datasets, perhaps against baseline methods that may already exist in that domain. The availability of labeled data, and especially the availability in analysis ready formats that might be readily ingested into a model training workflow, is the major impediment to uptake of advanced data analytics such as Deep Learning among the community of Earth surface scientists. While semi- or un-supervised classification methods are gaining more attention in many research contexts [Le et al., 2019] and are a staple method in landcover classification of mostly relatively 
Doodler: Human-in-the-Loop Segmentation of Earth Surface Imagery

coarse-resolution imagery [Smits and Dellepiane, 1997, Deng and Clausi, 2005], human annotators will continue to be vital for the success of many tasks that can be automated using ML. Despite the fact that the development of unsupervised methods require labeled data for development and, especially, evaluation, supervised methods at the time of writing are still state-of-the-art, and considered necessary to model imagery with high intra-class variance, such as a lot of Earth surface imagery. Supervised ML will therefore continue to be popular, and powerful, if facilitated by open-source tools that make data labeling more efficient, and analyses of uncertainty that add vital context to its use. Doodler, as what Monarch [2021] refers to as a 'smart interface for semantic segmentation,' is one of many specific software tools or interfaces [Bueno et al., 2020, Zhao et al., 2020, Goldstein et al., 2021] for the generation of large labeled datasets [Sumbul et al. 2019. Kashinath et al. 2021b] that can be used for teaching and self-exploration of Deep Learning techniques, for use in transfer learning, and for new model development. Doodler is an open-source program that runs in a web browser, and may be one of many similar future implementations that might use human-in-the-loop ML for efficient labeling of other scientifically relevant label data such as those generated from time-series signals or social media content [Cai et al., 2017].

The use of an ML model cascade, whereby the outputs of one classifier (MLP) is checked for consistency by another independent classifier (CRF), is crucial to the success of the approach for a wider variety of imagery and class sets. Image standardization, image feature engineering, spatial filtering, and the use of an ML model cascade all help reduce sensitivity of model outputs to user hyperparameters. These allow the human labeler to concentrate on annotating well, rather than spend time adjusting hyperparameters. We show that the proportion of the image pixels that require annotation for accurate pixelwise label image is relatively low around $10 \%$ of pixels for images of a size that is typically suitable for the program without excessive use of zoom and pan tools, which is imagery typically 3000 pixels in either horizontal dimension or less. Discrepancies in 
Doodler: Human-in-the-Loop Segmentation of Earth Surface Imagery

agreement are unavoidable with multiple labelers and represent a source of irreducible uncertainty in all image segmentation workflows. Doodler provides the means to rapidly label images, therefore multi-labeler label datasets are more readily acquired and the irreducible error can be quantified. Further, we show how combining agreement metrics can be used to flag inconsistent label images and annotation styles, and identify the effects of class imbalance. Dice and IOU scores are shown to be useful metrics for reporting agreement between segmentations of the same data by more than one labeler, and we recommend reporting mean agreement for multi-labeled datasets as an uncertainty and quality metric, per image, per class, or aggregated over images and/or classes. We also show how the metrics can be used to detect class imbalance, outlier labelers, and label images in multi-labeler contexts. Even though segmentations vary from person to person, that does not introduce unreasonable variance in label images created by different people, at different times, or using different computational infrastructure.

\section{Conclusions}

We describe a human-in-the-loop machine learning system involving a graphical user interface for fast, interactive segmentation of $\mathrm{N}$-dimensional $(\mathrm{x}, \mathrm{y}, \mathrm{N})$ images into two-dimensional $(\mathrm{x}, \mathrm{y})$ label images. It is designed to meet two objectives: 1) segmentation of relatively small datasets for specific geoscientific inquiries, and 2) segmentation of small to large amounts of imagery for subsequent training of other types of ML models for fully automated segmentation of large datasets. The program is designed to work with any type of Earth surface imagery. We demonstrate the approach using five case study datasets from river, estuarine, and open coast environments of the United States; 1) segmentation of beach sediments in visible-band aerial orthomosaic imagery to document change to beaches of Cape Cod, Massachusetts; 2) segmentation of post-hurricane aerial 
Doodler: Human-in-the-Loop Segmentation of Earth Surface Imagery

imagery from North and South Carolina, for assessment of storm impacts; 3) segmentation of aerial imagery for delineating complex shoreline environments; 4) segmentation of sidescan sonar imagery for mapping in-stream physical habitats in coastal plain rivers of Mississippi; 5) segmentation of false-color Sentinel-2 satellite imagery of coastal lagoon environments in Monterey, California, to study the dynamics of river breaching of beaches; and 6) segmentation of larger visible-band Landsat-8 satellite imagery of Cape Hatteras, North Carolina, to study coastal landform evolution at a regional scale. The datasets consist of irregular grids (each pixel does not represent the same spatial footprint), as well as regular grids. Based on comparitive exercises between hand-digitization using polygons and our alternative workflow, we conclude that our methodology encoded into the Doodler program is always faster, and also generally results in a segmentation that is as-or-more accurate than slower hand digitization workflows. We thereby demonstrate the effectiveness of the approach using geophysical, photographic, and multispectral imagery, as well as regular and irregular grids, and several different class sets and pixel sizes. The technique is reproducible in the sense that all decisions made by human labeler and ML algorithms (and their specific sequence) can be encoded to file, therefore the entire process can be played back and new outputs generated with alternative decisions and/or algorithms. We therefore expect our human-in-the-loop labeling workflow to have widespread applicability in Earth and Space scientific applications.

\section{Acknowledgments}

Data are available at https://datadryad.org/stash/share/7hUEqoIIsHEvTRu0_fXiQrc0skhPKKaFZRtdQ (note the final home for these data will be https://doi .org/10.5061/dryad.2fqz612ps). The cross-platform open-source web application is available athttps://github.com/dbuscombe-usgs / dash_doodler. This work has been supported by the U.S. Geological Survey Coastal/Marine 
Hazards and Resources Program and by Congressional appropriations through the Additional Supplemental Appropriations for Disaster Relief Act of 2019 (H.R. 2157). EBG acknowledges support from USGS (G20AC00403). CB acknowledges support from USFWS (F19AC00836). JF and SF acknowledge support from the USGS Community for Data Integration-funded Coast Train project. Thanks to Tanja Williamson, Meg Palmsten, and Chris Magirl for helpful suggestions. Any use of trade, firm, or product names is for descriptive purposes only and does not imply endorsement by the U.S. Government.

\section{Supporting Information}

\section{Fully Connected Conditional Random Field for Image Segmentation}

Starting with an unnormalized measure of the joint distribution given by [Koller and Friedman, 2009] $\widetilde{P}_{\Phi}(\mathbf{x}, y)=\prod_{i=1}^{I} \phi_{i}\left(D_{i}\right)$, where $\Phi=\left\{\phi_{i}\left(D_{i}\right), \ldots, \phi_{I}\left(D_{I}\right)\right\}, \phi_{i}$ are factors and $D_{i}$ are their associated scope, to model the conditional distribution $P(y \mid x)$, or the probability of a class $y$ given the image features $x$ is

$$
P(y \mid \mathbf{x})=\frac{1}{Z(\mathbf{x})} \widetilde{P}_{\Phi}(\mathbf{x}, y)
$$

where normalization constant $Z_{\Phi}(\mathbf{x})=\sum_{y} \widetilde{P}_{\Phi}(\mathbf{x}, y)$. Assuming the log probability of each class is a linear function of feature $x$ according to some model with parameters $\theta$, we model $\widetilde{P}_{\Phi}(x, y)$ as a Gibbs energy function, $E$, and the conditional distribution is rewritten

$$
P(y \mid \mathbf{x}, \theta)=\frac{1}{Z(\mathbf{x}, \theta)} \exp (-E(y \mid \mathbf{x}, \theta))
$$

Equation (2) is obtained following Krähenbühl and Koltun [2011] by summing unary $\left(\psi_{i}\left(y_{i}\right)\right)$ 
1017 and pairwise $\left(\psi_{i j}\left(y_{i}, y_{j}\right)\right)$ potentials:

$$
E(y \mid x, \theta)=\sum_{i} \psi_{i}\left(y_{i}, x_{i} \mid \theta\right)+\sum_{i \neq j} \psi_{i j}\left(y_{i}, y_{j}, \mathbf{f}_{i}, \mathbf{f}_{j} \mid \theta\right)
$$

where classes $i$ and $j$ range from 1 to $k$, pairwise potentials $\psi_{i j}\left(y_{i}, y_{j}\right)$ are the cost of simultaneously assigning label $y_{i}$ to grid node $i$ and $y_{j}$ to grid node $j$ and are detailed below, and $\psi_{i}\left(y_{i}\right)$ are unary potentials, computed as:

$$
\psi_{i}\left(y \mid x_{i}\right)=-\log \left(P\left(y \mid \theta, x_{i}=y_{i}\right)\right)
$$

in which $P\left(y \mid \theta, x_{N}=i\right)$ is the likelihood of location $N$ being class label $i$, based on the extracted feature vector at that location, which can be computed for each pixel location using a classifier model that has approximately captured the relationship between the label and image data. The vectors $\mathbf{f}_{i}$ and $\mathbf{f}_{j}$ are features created from $x$. Here, $\mathbf{f}_{i}$ and $\mathbf{f}_{j}$ are controlled by pairwise potentials $\psi_{i j}\left(y_{i}, y_{j}\right)$ and are therefore a function of both the relative position as well as amplitudes of the image features. Minimizing Equation (3) yields the most probable label assignment, whereby the maximum a posteriori (or MAP) for the labeling $(y \in k)$ is $y^{*}=\arg \max _{y \in k} P(y \mid \theta, x)$, which chooses what is the most likely $y$ considering $x$. Features $x$ are mapped to graphs, where each datum represents a graph node, and every node is connected with an edge to its neighbors according to a connectivity rule.

The pairwise potential $\psi_{i j}\left(y_{i}, y_{j}, \mathbf{f}_{i}, \mathbf{f}_{j} \mid \theta\right)$ encodes the joint likelihood that the pair of pixel locations $i$ and $j$ are assigned class labels $y_{i}$ and $y_{j}$, respectively, based on the similarity of feature vectors from respective pixel pair locations, as well as their relative proximity in image space, normalized by the average difference between feature vectors over all the adjacent pixels in the image, with degree of adjacency in feature and image space controlled by hyperparameters. Where 
$l$ denotes feature vector derived from $\mathbf{x}$,

$$
\psi_{i j}\left(y_{i}, y_{j}, \mathbf{f}_{i}, \mathbf{f}_{j} \mid \theta\right)=\Lambda\left(y_{i}, y_{j} \mid \theta\right) \sum_{l=1}^{L} k^{l}\left(f_{i}^{l}, f_{j}^{l}\right),
$$

where each $k^{l}$ is a function that determines the similarity between connected grid nodes by means of an arbitrary feature $f^{l}$. The function $\Lambda$ quantifies label 'compatibility', by imposing a penalty for nearby similar grid nodes that are assigned different labels. Pairwise potentials (5) are computed as linear combinations of Gaussian kernels Krähenbühl and Koltun [2011]:

$$
k^{l}\left(f_{i}^{l}, f_{j}^{l}\right)=\exp \left(-\frac{\left|\mathbf{x}_{i}-\mathbf{x}_{j}\right|^{2}}{2 \theta_{\beta}^{2}}\right)+\exp \left(-\frac{\left|p_{i}-p_{j}\right|^{2}}{2 \theta_{\gamma}^{2}}\right)
$$

where $p_{i}$ and $p_{j}$ are grid positions. The first kernel quantifies the observation that nearby grid nodes with similar image features are likely to be in the same class. The degree of similarity is controlled by the hyperparameter $\theta_{\beta}$ (non-dimensional). As $\theta_{\beta}$ increases, larger differences on the $l$-th feature are tolerated. The second kernel removes small isolated regions; that final CRF hyperparameter is held constant in the Doodler implementation and therefore is not tunable by the user. This is due to concerns of exposing too many parameters, and this one generally has relatively limited effect compared to the other two. We use $\theta_{\gamma}=3$ to extract spatial features to map to classes. We use a relatively small $\theta_{\gamma}$ to encourage the model to assign the same class to image pixels separated by relatively small distances, imposing a larger numerical penalty for classes separated by larger distances. In some imagery, there is a strong spatial gradient in the distribution of classes across the scene, in which case a relatively small $\theta_{\gamma}$ would discourage the model assigning a particular class to small islands of pixels in distal locations to other example pixels of that class. Our implementation therefore limits the success of looking for very small linear or 'island' features. In other situations where relative location is a weak predictor of class, small $\theta_{\gamma}$ acts to not discourage the assignment of 
a class in a particular location, but might lessen high-frequency (i.e. speckle) noise in the estimated label image.

\section{Image Standardization and Feature Extraction}

Each input image, $I(i, j, d)$, where $i$ and $j$ describe 2D pixel locations and $d$ indicates the number of coincident data layers, is standardized by

$$
I_{S}(i, j, d)=\frac{I(i, j, d)-\mu_{I}}{\sigma_{a d j}}
$$

where $\mu_{I}$ is the global mean of $I(i, j, d)$, and $\sigma_{a d j}$ is the adjusted standard deviation of $I(i, j, d)$, computed as $\max \left(\sigma_{I}, 1 / \sqrt{N}\right)$ where $\sigma_{I}$ is the global standard deviation of $I(i, j, d)$ and $N=$ $\sum(i) \sum(j)$ is the number of pixels.

Image intensity features $I_{f}(i, j)$ are extracted from $I_{s}(i, j, d)$ by convolving with filter bank $\Sigma_{s}$, or $I_{f}=\Sigma_{s} * I_{s}$ where $*$ denotes convolution, and where $\Sigma_{s}$ consists of $s$ D Gaussian kernels, each defined as

$$
G\left(K_{i}, K_{j}\right)=\frac{1}{2 \pi \sigma_{s}^{2}} e^{-\frac{K_{i}^{2}+K_{j}^{2}}{2 \sigma_{s}^{2}}}
$$

where $K_{i}$ and $K_{j}$ are the respective distances from the origin in the horizontal and vertical axes of the kernel, $\sigma_{s}$ is one of a user-defined number of different values of standard deviation of the Gaussian distribution, distributed logarithmically between 0.5 and 16 (units are pixels). 
1071

1072

1073

1074

1075

1076

1077

1078

1079

1080

1081

1082

1083

1084

1085

1086

1087

1088

1089

\section{Multilayer Perceptron}

The feature stack used for initial segmentation consists of a set of 3D $(i, j, d)$ grids, each flattened to $1 \mathrm{D}(1, i j d)$, then stacked columnwise to create the input vector

$$
\mathbf{x}=\left[L(i j d), I_{f}(i j d), \nabla_{I_{f}}(i j d), H_{1}(i j d), H_{2}(i j d)\right]
$$

The standard Multilayer Perceptron or MLP model is solved as linear combination of single layer perceptron units each with their own weights $w$ and biases $b$, represented algebraically as

$$
f=\Phi\left(\mathbf{w}^{T} \mathbf{x}+\mathbf{b}\right)
$$

where $\mathbf{w}$ and $\mathbf{b}$ denote the matrices of weights and biases, respectively, consisting of vectors from all hidden layers, that the model learns during a brief training period, and $\Phi(x)=\max (0, x)$ is the rectified linear unit activation function.

Whereas there is no drop-in replacement for the CRF, the MLP could be switched to a different ML framework. In fact, we have also extensively trialled a Random Forest model framework but decided that the MLP performed better; see Figure 15 for an example, based on dataset A.

\section{Spatial Filtering of Initial Segmentation}

The first filter (Figure $25 \mathrm{a}-\mathrm{d}$ ) creates a one-hot encoded stack from the label image (Figure 25b), $m(i, j, d)$, that is a $i j k$-dimensional matrix encoding the occurrence of each pixel $i, j$ and each class $k$, i.e. a binary $2 \mathrm{D}$ matrix of zeros and ones for each of $k$ classes. For each binary image in the stack, small 'holes' of zeros within large areas of ones are assumed to be erroneous, and filled in with ones, using an area threshold. Similarly, 'islands' of ones less than the same threshold area 
Doodler: Human-in-the-Loop Segmentation of Earth Surface Imagery

1090

1091

1092

1093

1094

1095

1096

1097

1098

1099

1100

1101

1102

1103

1104

1105

1106

1107

1108

1109

are removed (filled in with zero). Those pixels where the entire one-hot stack is now zero are then reclassified using the second-most likely class, based on the probabilities estimated by the MLP. The reader is referred to Figure 26 for another example workflow.

The second filter (Figure 26-f) determines a null class to allow the CRF model to estimate the appropriate class values for pixels that are furthest away from similar classes, based on some threshold distance. Those pixels occur at the transition areas between large contiguous regions of same-class. The filter based on the 2D map of Euclidean distances between pixels of similar class (i.e. ones) in each binary $2 \mathrm{D}$ matrix in $m(i, j, d)$, is given by

$$
D_{\mathbf{i}}(m)=\sqrt{\left(\sum_{\mathbf{i}}\left(m_{\mathbf{i}}-b_{\mathbf{i}}\right)^{2}\right)}
$$

where $b_{\mathbf{i}}$ is the background point (value 0$)$ at point $\mathbf{i}=(i, j)$ with the smallest Euclidean distance to input points $m_{\mathbf{i}}$. The filter is based on the 2D map of Euclidean distances between pixels of similar class (i.e. ones) in each binary 2D matrix in $m(i, j, d)$, denoted by $D_{\mathbf{i}}(m)$. The pixel locations are zeroed where values of $D_{\mathbf{i}}(\mathrm{m})$ are less than a threshold (default is two pixels), the application of which results in a thin transition region of two zero pixels between each region of different-valued classes. The one-hot encoded matrix is then converted back to a final $2 \mathrm{D}$ label image using the argmax function, that is used as inputs to the final CRF model. That means there will be no ones in any $k$ class at the filtered $i, j$ locations; the intent of zeroing these pixels is to define a 'null class' to allow the CRF model to estimate the appropriate class values for pixels in those spatially small and isolated areas. Therefore the set of classes given to the CRF model is zero, plus the set of $k$ classes annotated. The reader is referred to Figure 27 for an example workflow. 
Doodler: Human-in-the-Loop Segmentation of Earth Surface Imagery

\section{Doodler Program Implementation}

The Doodler program consists of a few Python scripts that use Dash Dash [2021] for the web application and Flask Grinberg [2018] as the back-end web server. Dash is an interactive, opensource, browser-based graphing library built on Plotly.js Plotly [2015] and React React [2021]. It runs either as a command line program using dependency libraries installed within a virtual environment, or from a Docker container Merkel [2014] for deployment on any platform. Dash provides an API for Plotly libraries in R, and Julia, which are popular scripting languages among Earth scientists, meaning the web application code could be ported to those languages relatively easily. Alternatively, the web application for gathering label data could be written in any one of a number of different modern web application frameworks such as React React [2021] or Holoviz Holoviz [2021]. Therefore here we only document the essential generic features of the application that could be reproduced readily in an alternative platform.

Users prepare their own imagery for input to the program; if more than three coincident bands exist (in real-world or more generally in image coordinates), a three-band combination for optimal classification must be determined beforehand, and the 2D label would be assumed to apply to all $N$ coincident bands. Classes are created/edited using a text file to be read into the program, which automatically assigns colored buttons for each class. Numerical implementation of our methods relies heavily on the scikit-learn library Pedregosa et al. [2011] that facilitates implementation of a model for estimating unary potentials, such as a Multilayer Perceptron or Random Forest or any common discriminative model, as well as the numpy library Harris et al. [2020], and results are written to the compressed numpy format, npz, that provides storage of array data using gzip compression. This format is non-proprietary, and while it has no metadata fields, it serves well as a data storage option for ML model frameworks trained on Graphics Processing Units, like most 
modern ML frameworks, because it is a platform- (but not language-) agnostic and extendable option for serializing structured data like image-label pairs. All iterations of the sparse annotations and subsequent label image estimates are saved to file, along with all user settings. It is therefore possible to reconstruct any label image from the sparse annotations, with the original hyperparameters or another set. A log file keeps track of every button press by the user. Annotations are rasterized from Scalable Vector Graphics (SVG) but could be easily modified to remain in SVG format if vector outputs are required.

\section{Comparison of Segmentations}

The mean Intersection over Union is given by

$$
I O U=\frac{1}{k} \sum_{k} \frac{\left|Y_{k} \cap \widehat{Y_{k}}\right|}{\left|Y_{k} \cup \widehat{Y}_{k}\right|}
$$

and is estimated for each label image per class, then averaged over $k$ classes, where $Y$ and $\widehat{Y}_{k}$ are first and second label images for the $k$ th class, respectively, $\cap$ is intersection, and $\cup$ is union Costa et al. [2018].

$$
D=\frac{1}{k} \sum_{k} \frac{2\left|Y_{k} \cap \widehat{Y_{k}}\right|}{\left|Y_{k}\right|+\left|\widehat{Y}_{k}\right|},
$$

Mathematically, Dice is equivalent to an F1 score, the harmonic mean of precision and recall Haque and Neubert [2020]. It can be shown that $D \geq I O U$; the two functions are maximally divergent when either is at 0.5 (Figure 17), when the average denominator in either Equation (12) or (13) is twice as large as the average numerator. 


\section{Data Set}

1152 The dataset used in this study, consisting of a single zipped file containing 7 folders: 1) dataset A, 2)

1153 dataset B, 3) dataset C, 4) dataset D, 5) dataset E, 6) dataset F. In each folder are subfolders 1) images,

1154 2) label images, 3) annotations. The images folder contains the raw images used to generate label 1155 images using the program, another folder contains the label images generated by the program, and 1156 the annotations folder contains the raw annotations. All images are in standard image formats jpeg 1157 and png. It will eventually be available from https://doi.org/10.5061/dryad.2fqz612ps. 

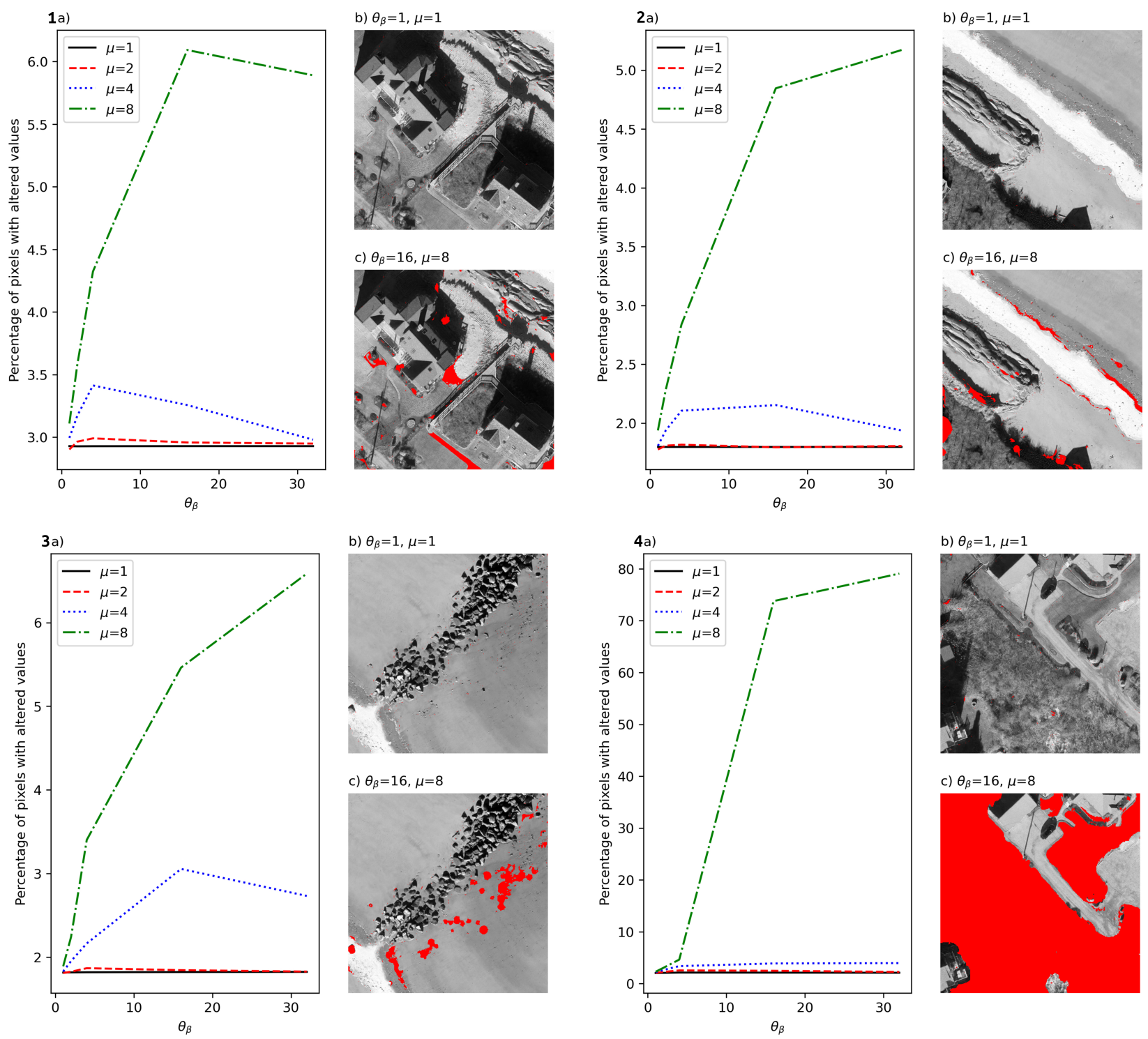

Figure 14: An illustration of the effects of varying $\theta_{\beta}$ and $\mu$ on four example images from the Sandwich Town Beach dataset, numbered 1 through 4 . In each, a) shows the percentage of pixels relabeled by the CRF, as function of $\theta_{\beta}$ and $\mu$, not including the pixels reclassified by spatial distance transform; $b$ ) illustrates the location of relabeled pixels when $\theta_{\beta}=\mu=1$ (there may be so few they are hard to see); and c) illustrates the location of relabeled pixels when $\theta_{\beta}=16$ and $\mu=8$. 

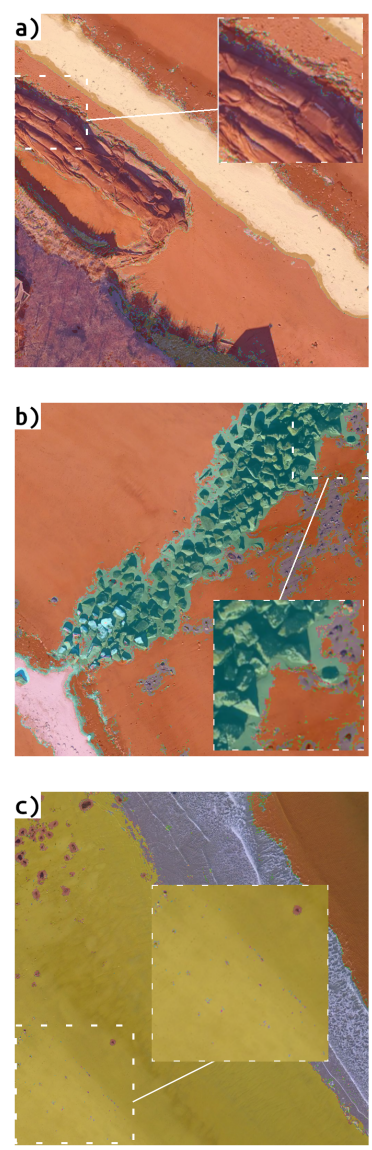
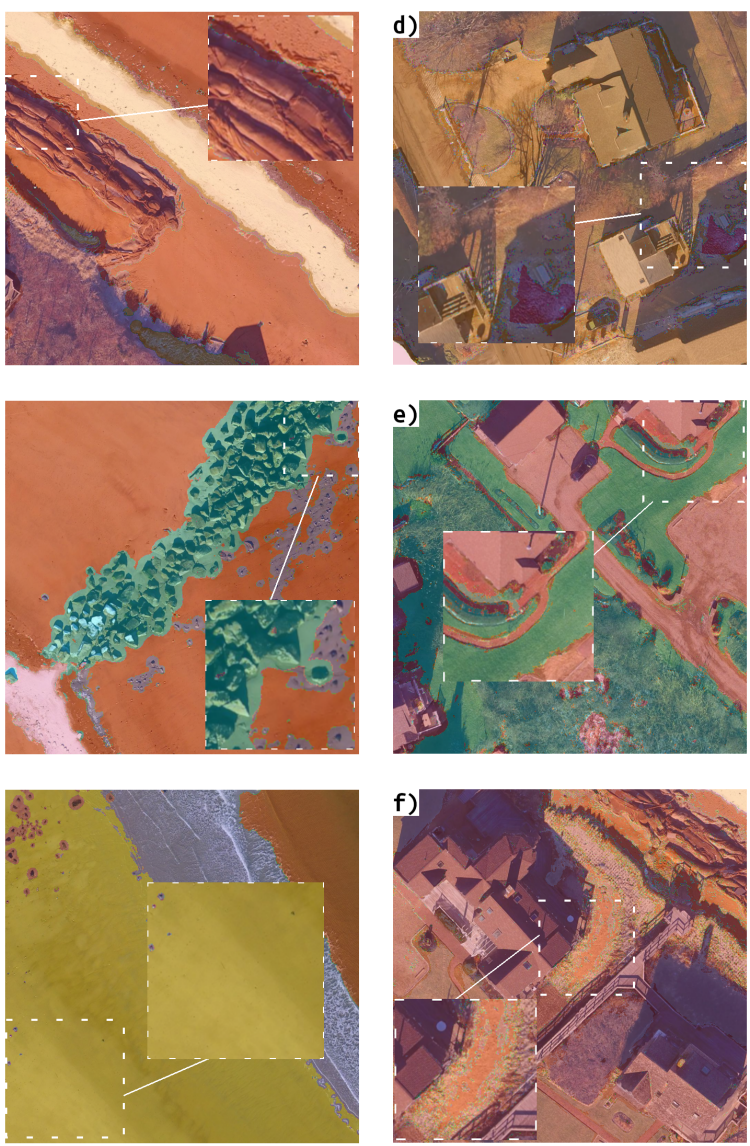
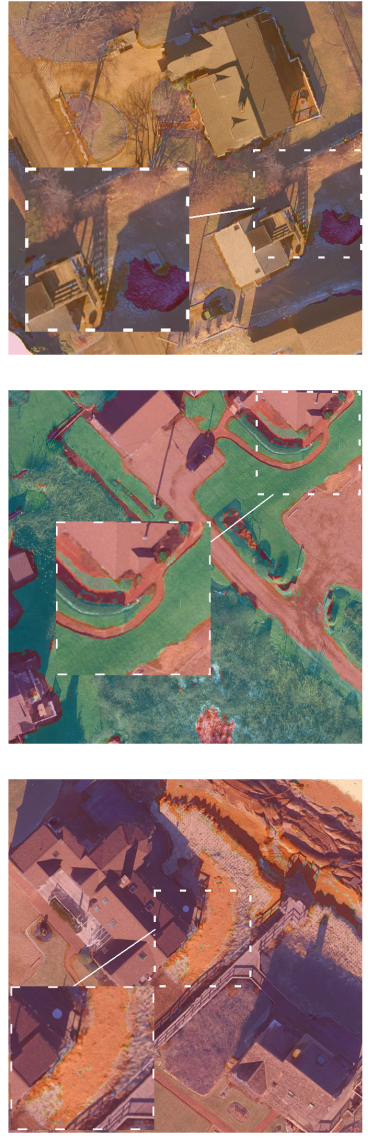

Figure 15: A comparison of the label images estimated from sparse annotations ('doodles') by two different discriminative ML model frameworks, namely the Random Forest (RF) and Multi Layer Perceptron (MLP). The six example comparisons shown come from the Sandwich Town Beach dataset; in each, the original image tile is superimposed with a semi-transparent overlay of the color label image. In each case, the RF outputs are on the left and the equivalent MLP outputs on the right. In each case, the two models perform almost equally well, however the RF outputs systematically have more error at or near the pixel level, i.e. high-frequency noise of small, spatially isolated mispredictions, compared with the MLP outputs. Our implementation therefore uses the MLP, however, a RF could be considered a stand-in replacement for the MLP in certain cases. 

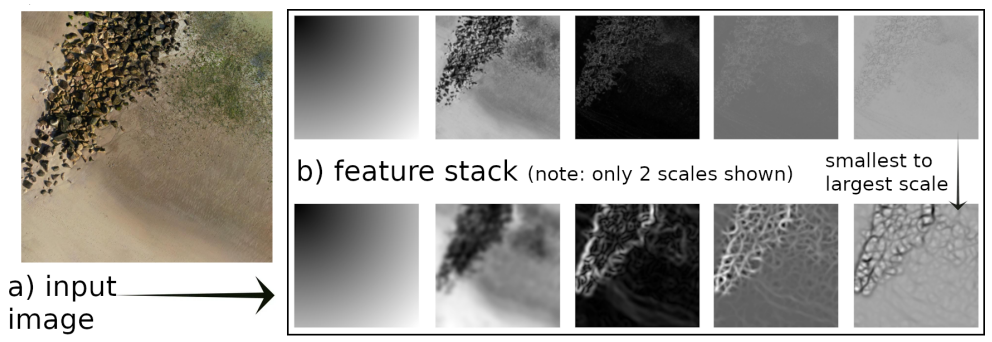

image

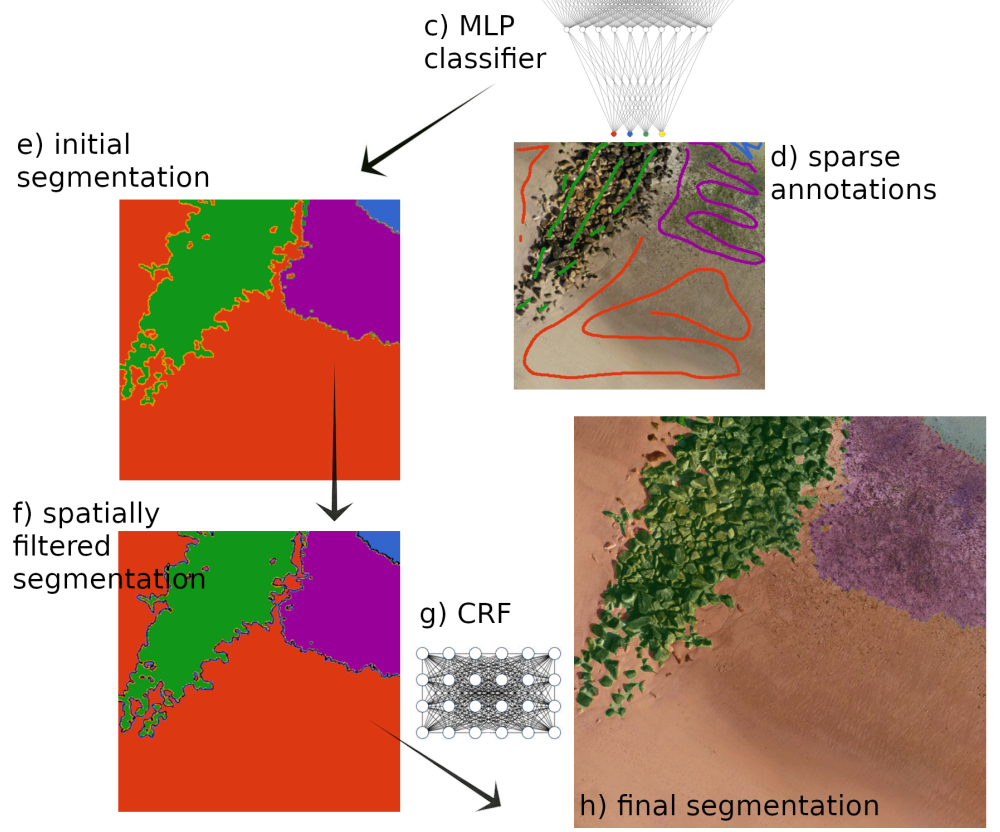

Figure 16: An illustration of the full workflow, using one tile of dataset A (Sandwich beach). From the original image (a), a set of feature maps or 'feature stack' '(b) are extracted, consisting of five features extracted using kernel convolution methods (location, intensity, edges, minimum curvature and maximum curvature) each computed over up to 15 scales (decided by the user in our implementation, the program Doodler). Note that b) only shows the first five and the last five feature maps, i.e. those extracted with the smallest and largest sized kernels, respectively. The feature stack is used to train a MLP classifier (c) to learn from sparsely annotations provided with strokes of a mouse or stylus (d) with examples of each class in each region of the image that the class exists. The MLP model output is an initial segmentation (e), which us spatially filtered (f) using the one-hot label method shown in Figure 26, then spatially filtered using the distance method shown in Figure 27, leaving a null class (e; black shows the null or zero pixels). Finally, the CRF model ( $\mathrm{g}$ ) is used to provide the final label image by evaluating the likelihood of the MLP solution and making adjustments acoordingly. The image shown in $h$ ) is the final label as a semi-transparent overlay of the original input image, showing very close agreement. 


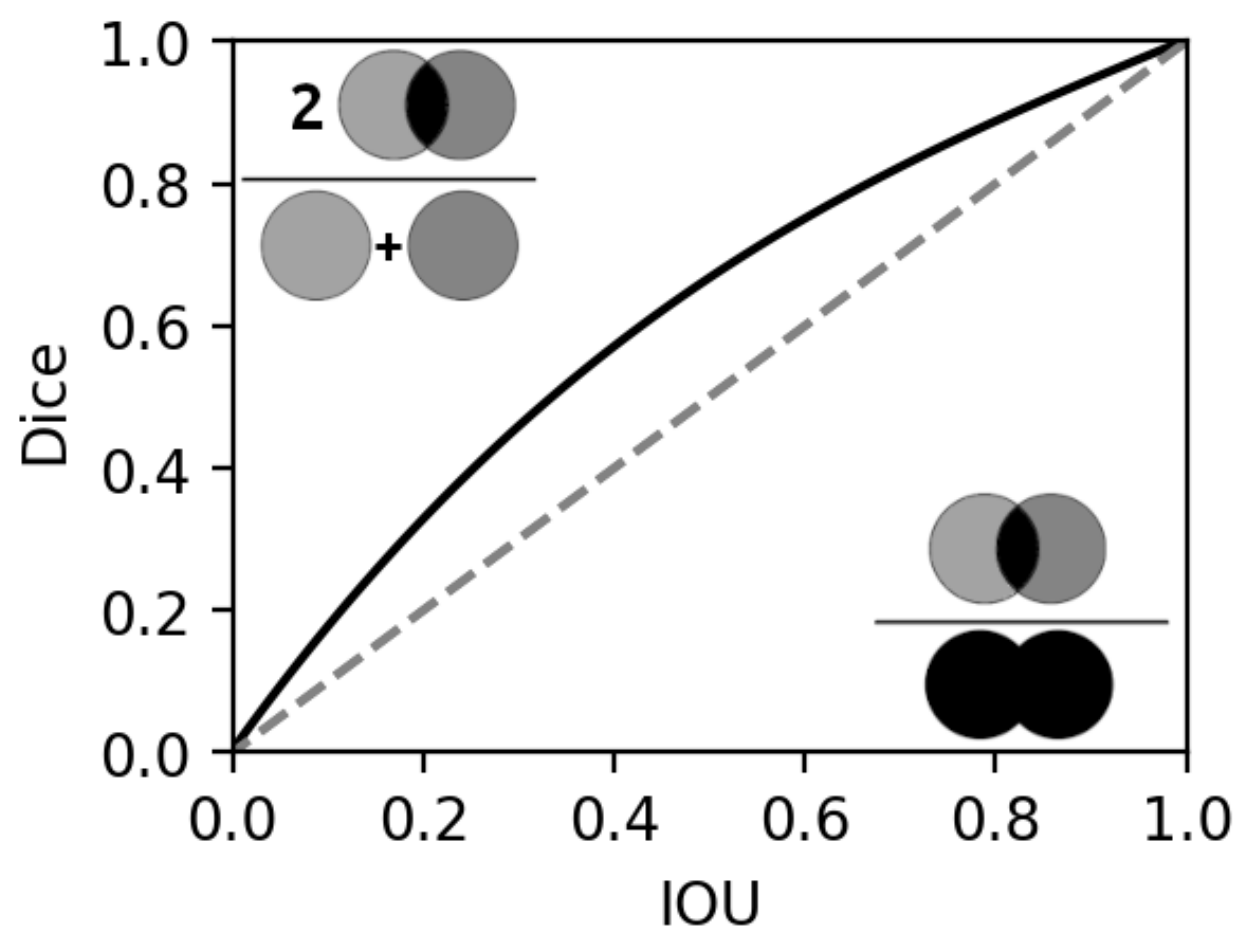

Figure 17: The functional relationship between mean Dice and mean Intersection over Union (IOU; solid line) scores, with the 1:1 (dashed line) and a cartoon of each metric for reference. 

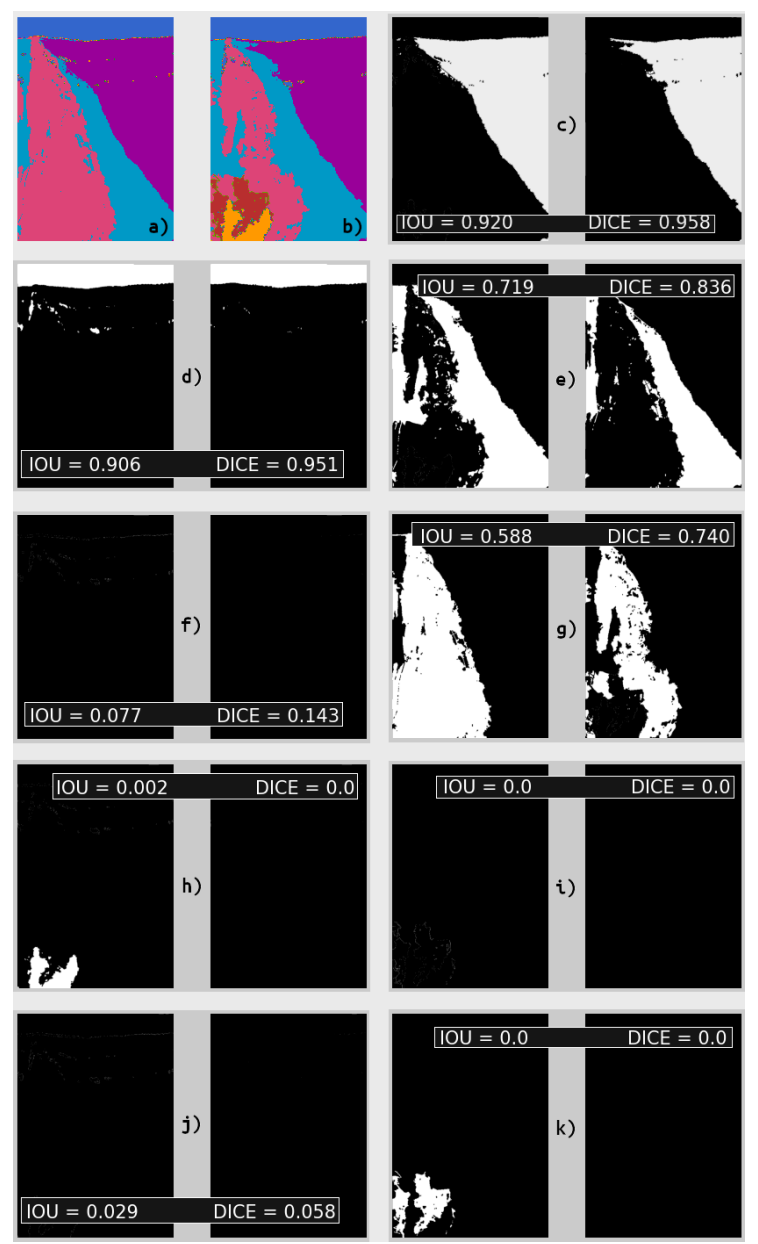

Figure 18: A visual illustration of the quantification of the mean IOU and Dice scores for a pair of label images ( $\mathrm{a}$ and $\mathrm{b}$ ). The mean IOU between these two label images is 0.36 and the mean Dice is 0.41. Scores are relatively low because there are two additional classes in (b) as there are in (a) that each represent a significant proportion of the image. In each pair of plots c) through $\mathrm{k}$ ), the left is the union and the right is the intersection of the two label images for a particular class. For each, the per-class IOU is reported. There is a high agreement for the fine bedforms (c), water (d) and coarse (e) classes, and a reasonable agreement between the remaining major class for this image, namely coarse sediment (g). However, the two classes in (b) not present in (a) have negligible Dice (h and $\mathrm{k}$ ), which considerably lowers the average scores. A further three classes ( $\mathrm{f}, \mathrm{i}$ and $\mathrm{j}$ ) are revealed to be present in (b) but not (a), but each represent just a few pixels and further decreases the scores. 


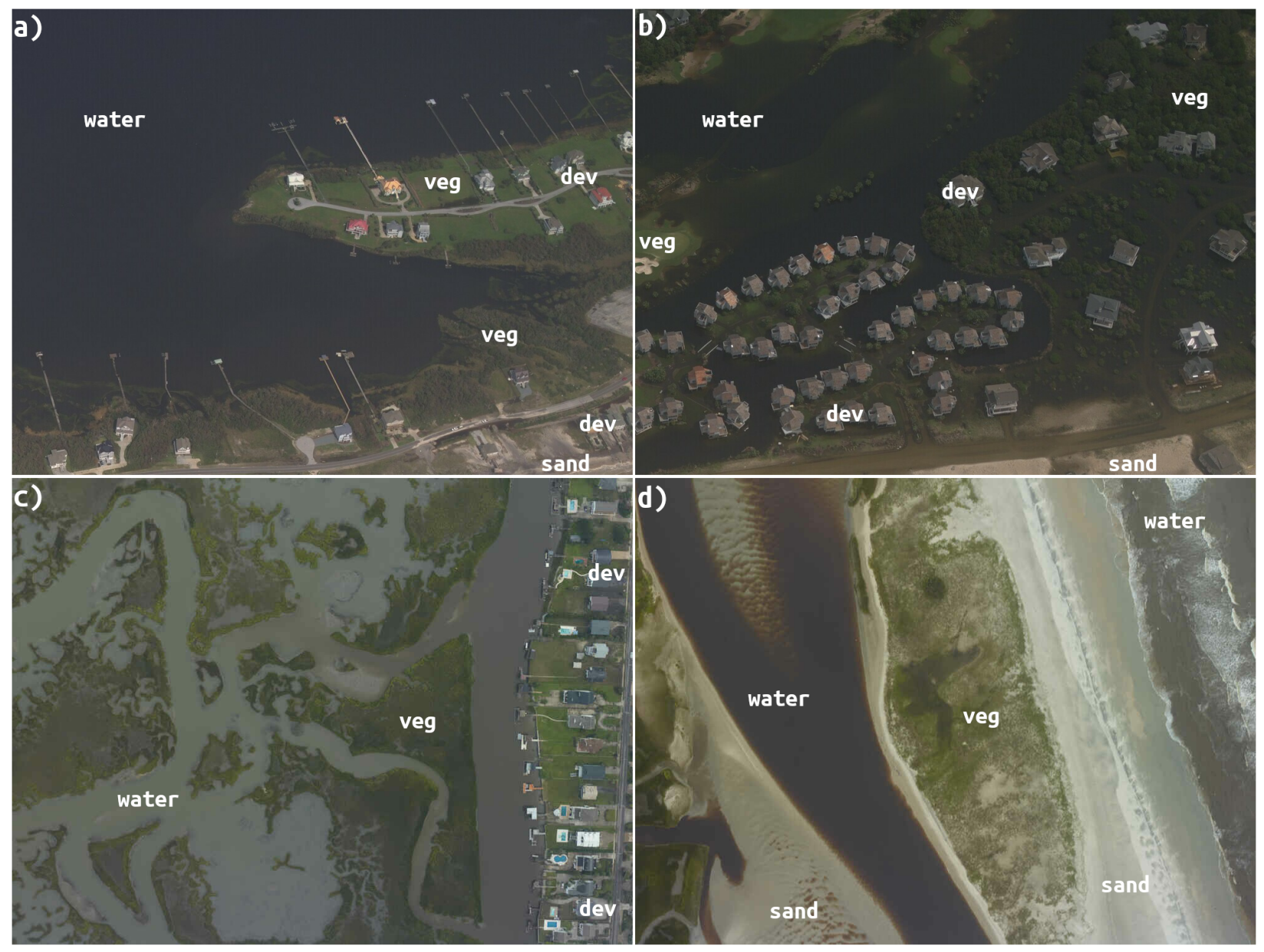

Figure 19: Four example post-hurricane aerial photographic images, each showing examples of each class. 


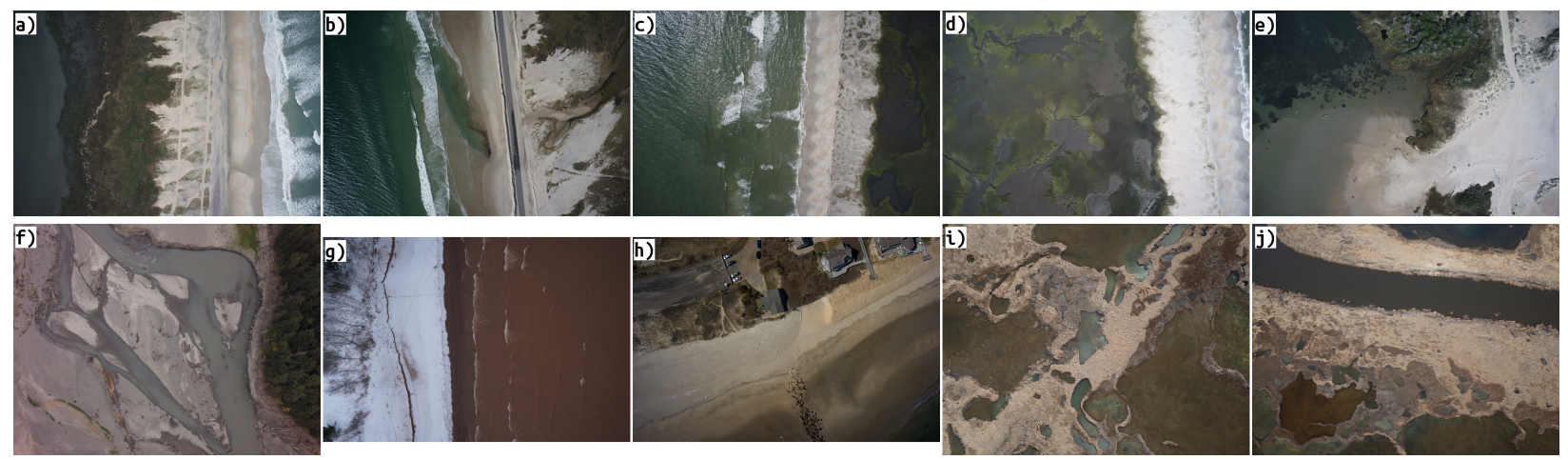

Figure 20: All ten images in the second aerial dataset. Notice that some scenes are open coast, and other are of estuarine and wetland environments. In each case, the image has been selected to be difficult, containing shallow areas that are ambiguous to delineate and define.

\section{References}

Tom G Farr, Paul A Rosen, Edward Caro, Robert Crippen, Riley Duren, Scott Hensley, Michael Kobrick, Mimi Paller, Ernesto Rodriguez, and Ladislav Roth. The shuttle radar topography mission. Reviews of Geophysics, 45(2), 2007.

Noel Gorelick, Matt Hancher, Mike Dixon, Simon Ilyushchenko, David Thau, and Rebecca Moore. Google Earth Engine: Planetary-scale geospatial analysis for everyone. Remote Sensing of Environment, 202:18-27, 2017.

Michael A Wulder, Thomas R Loveland, David P Roy, Christopher J Crawford, Jeffrey G Masek, Curtis E Woodcock, Richard G Allen, Martha C Anderson, Alan S Belward, and Warren B Cohen. Current status of Landsat program, science, and applications. Remote Sensing of Environment, 225:127-147, 2019.

Wolfgang Schwanghart and Dirk Scherler. TopoToolbox 2-MATLAB-based software for topographic analysis and modeling in Earth surface sciences. Earth Surface Dynamics, 2(1):1-7, 2014.

Andrew D Richardson, Koen Hufkens, Tom Milliman, and Steve Frolking. Intercomparison of phenological transition dates derived from the PhenoCam Dataset V1. 0 and MODIS satellite remote sensing. Scientific Reports, 8(1):1-12, 2018.

Ian J Walker, Robin GD Davidson-Arnott, Bernard O Bauer, Patrick A Hesp, Irene DelgadoFernandez, Jeff Ollerhead, and Thomas AG Smyth. Scale-dependent perspectives on the geomorphology and evolution of beach-dune systems. Earth-Science Reviews, 171:220-253, 2017. 

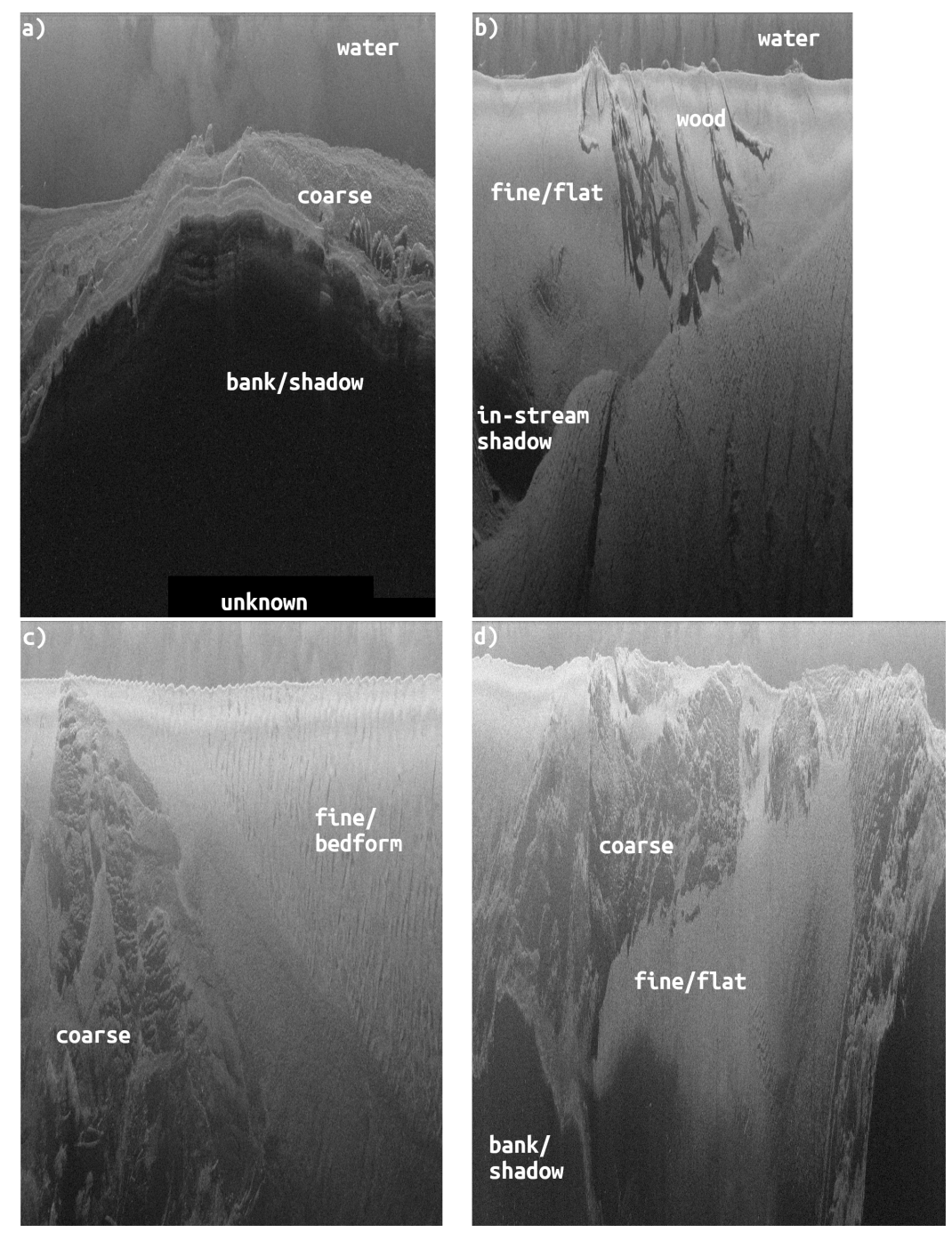

Figure 21: Four example sidescan images, each with some classes identified. 

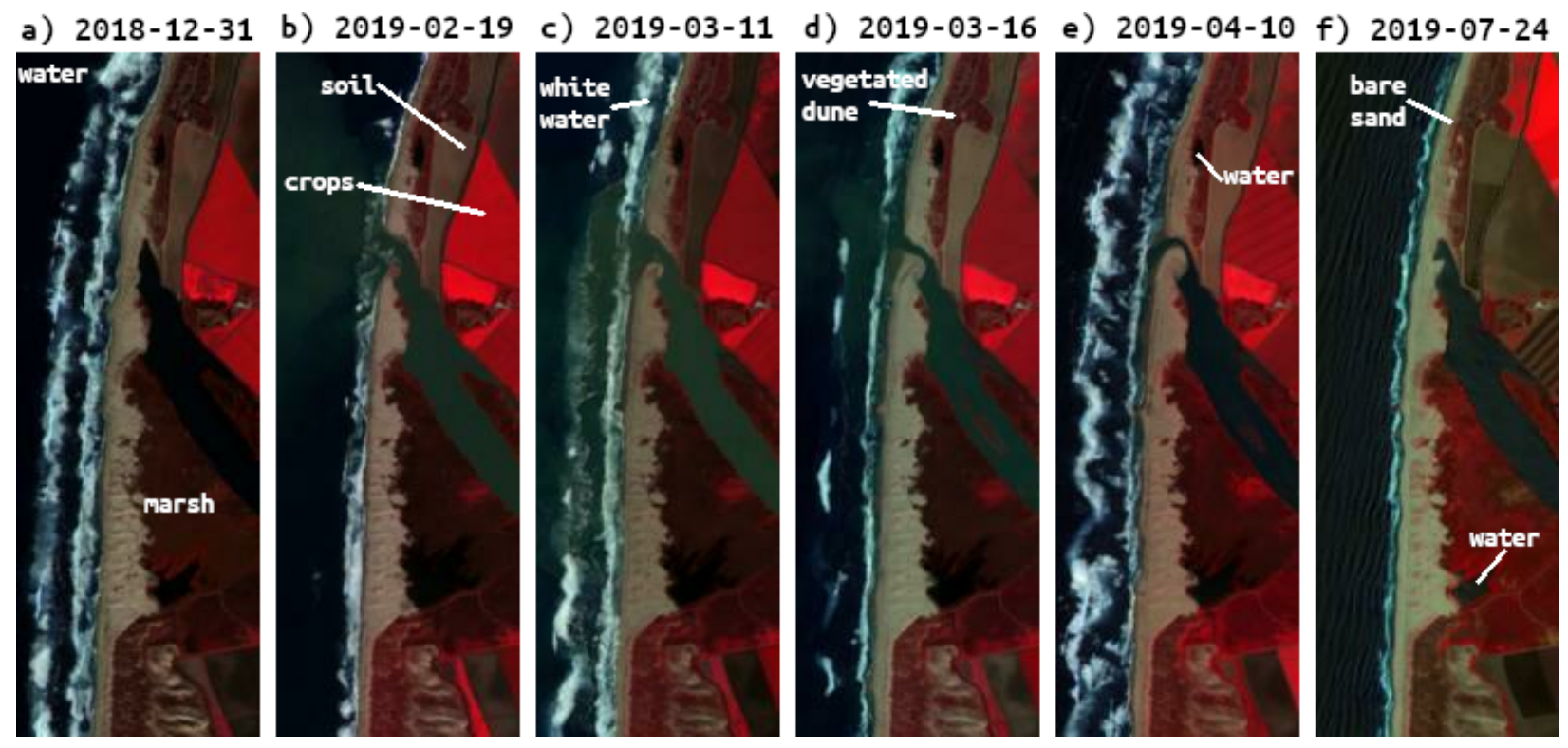

Figure 22: A time-series of false-color Sentinel-2 images consisting of band 8 (near infrared), red (band 4), and green (band 3). These examples span the period Feb 15, 2015 and Sept 27, 2021 during which time a breaching event occurred and subsequently the barrier resealed, as is visible in the imagery. Some classes are also identified. 
Doodler: Human-in-the-Loop Segmentation of Earth Surface Imagery
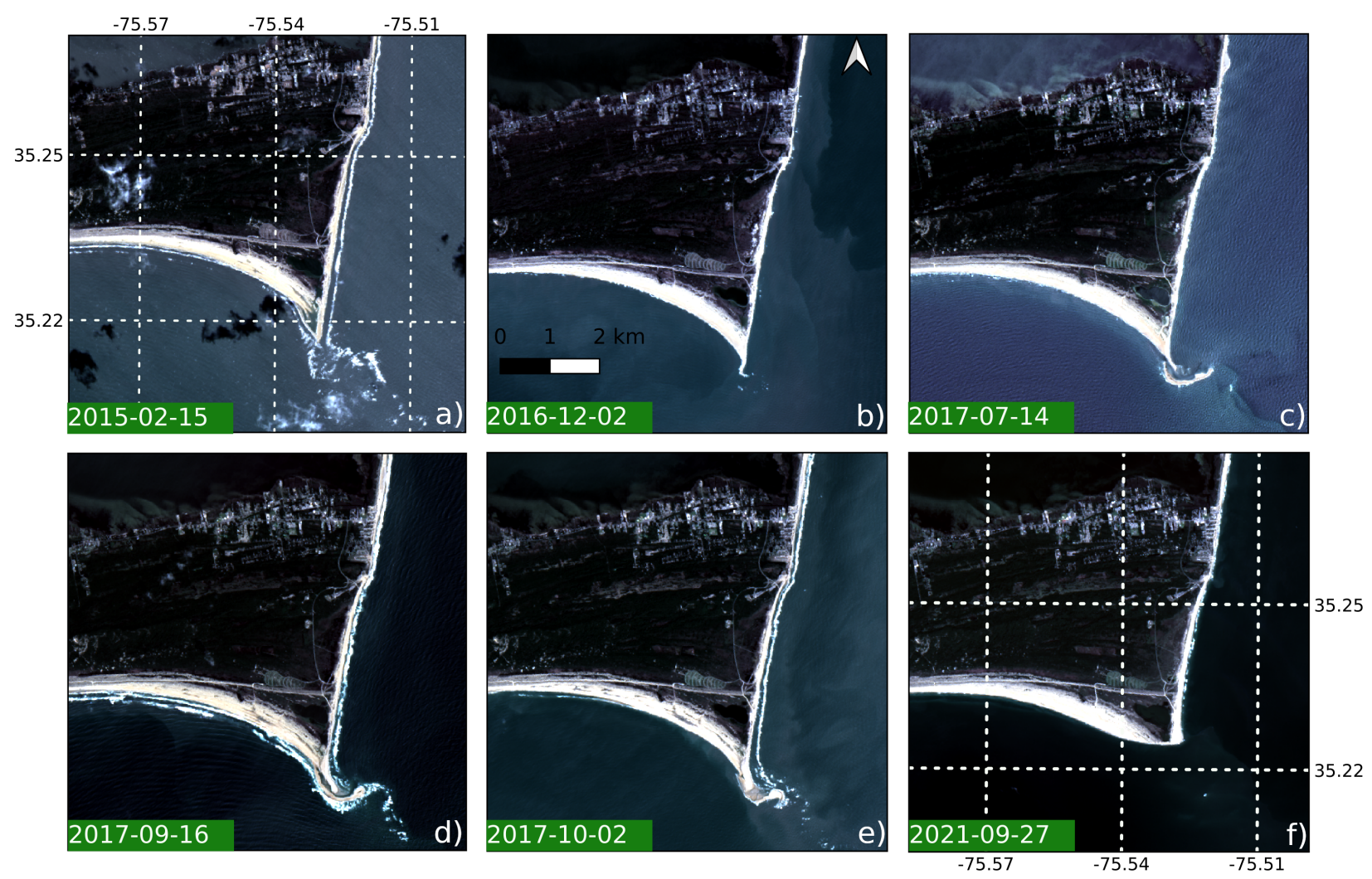

Figure 23: A time-series of visible-band Landsat-8 images of Cape Hatteras National Seashore, in North Carolina, USA. These examples span the period 2nd February, 2015 and September 27th, 2021. Initially (a), there is an onshore-migrating bar that by late in 2016 (b) had welded onto shore and formed a spit. By summer of 2017 (c), another bar (this one of crescentic shape) had formed spanning the cape, which by September of 2017 (d) had welded to shore, then breached by October 2nd (e). Since that time, the cape has been in steady recession, such that by September 2021 the cape is farther north and east than at any point since at least early 2015. 

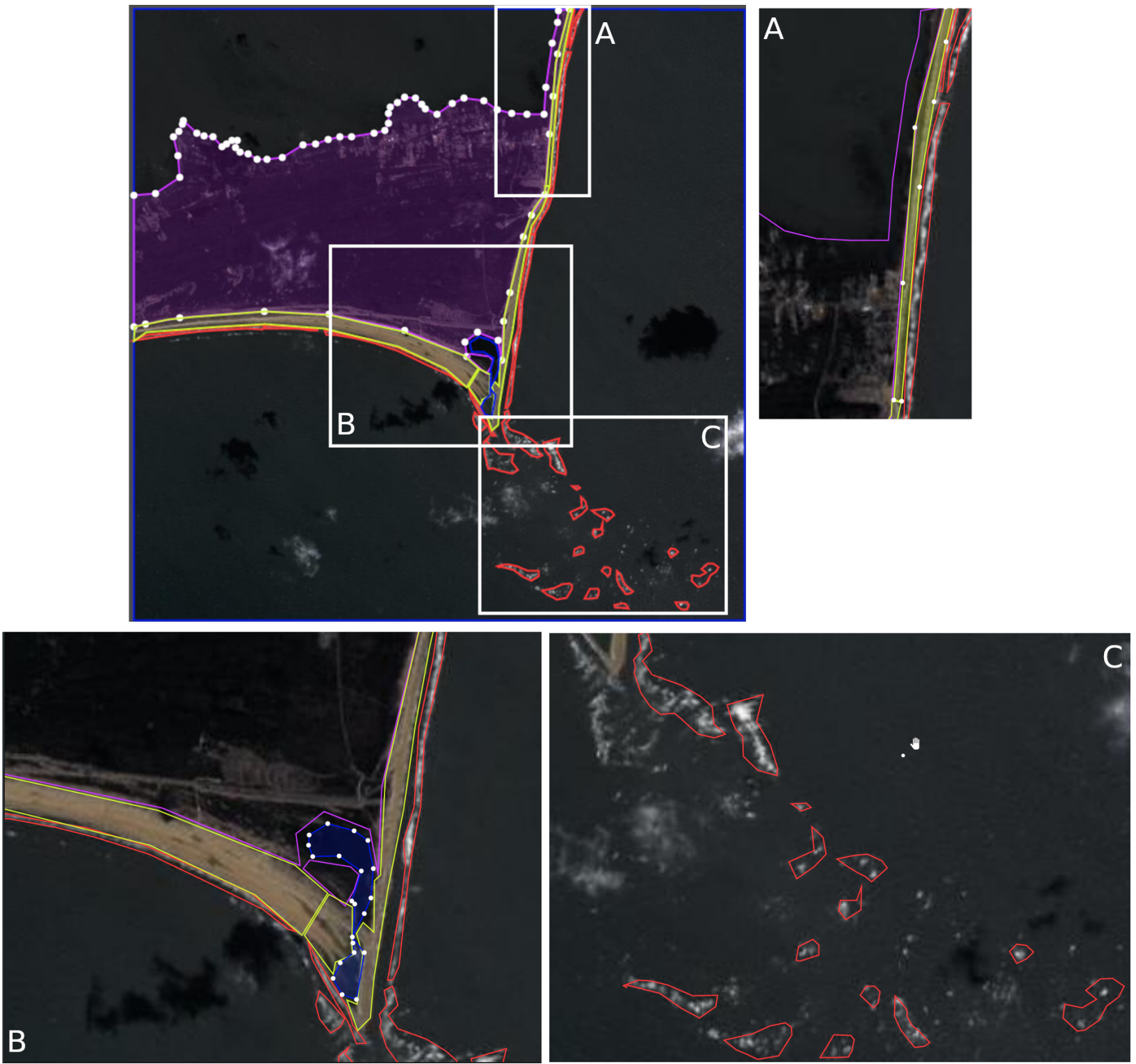

Figure 24: Hand annotation workflows using the Makesense.ai program Skalski [2019]. The level of zoom and pan required to effectively label such large scenes is comparable between Doodler and Makesense.ai and other programs that facilitate labeling by hand-drawing polygons. 


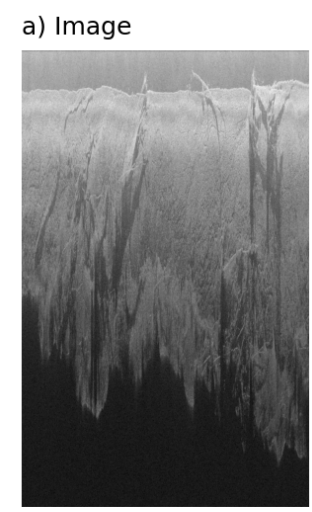

c) Spatially filtered label image

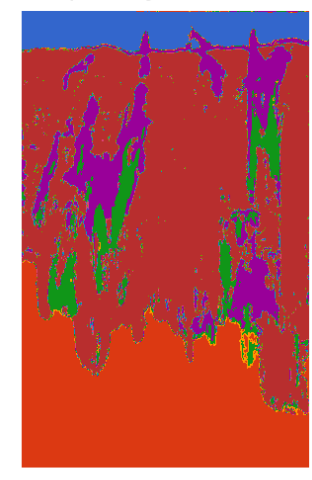

e) Distance-filtered label image

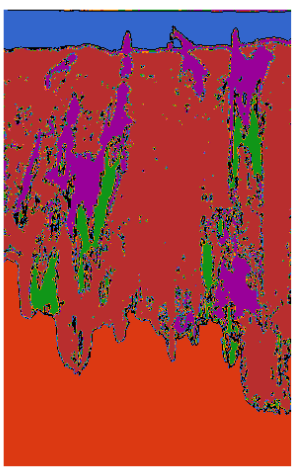

b) Label image

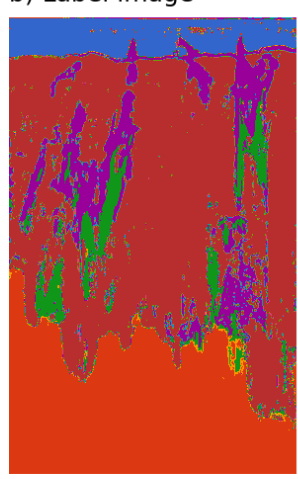

d) Reclassified pixels

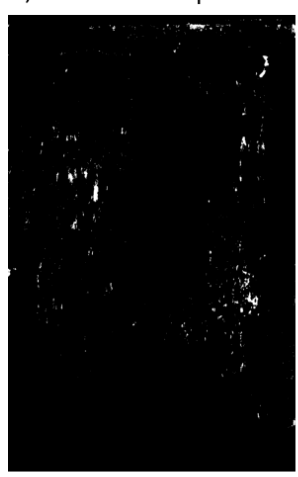

f) Pixels for CRF reclassification

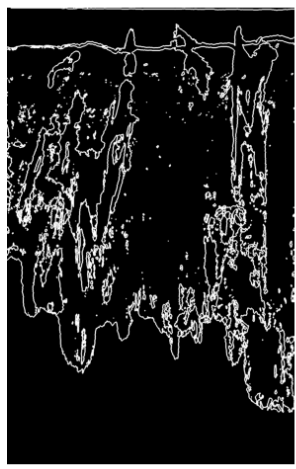

Figure 25: The two-stage label image filtering process; a) the raw image, b) the label image (unary potentials) produced by the Multilayer Perceptron (MLP) classifier; c) the spatially filtered label image; d) the pixels that are reclassified by the spatial filtering (stage 1); e) the label image as a result of the distance-based filtering with an additional null (zero) class shown as black pixels (stage 2); and f) those null pixels identified that will be reclassified by the Conditional Random Field (CRF) model. Subplot $\mathrm{f}$ ) is redundant of e) but is used for visual comparison of the relative number of pixels reclassified as a result of spatially filtering the one-hot encoded label stack (d) and the distance filter (f). 
a) Image

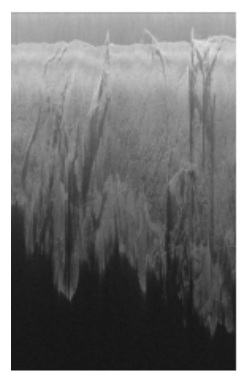

c) Class 1

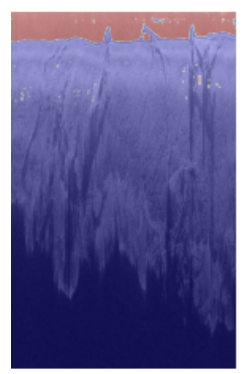

e) Class 2

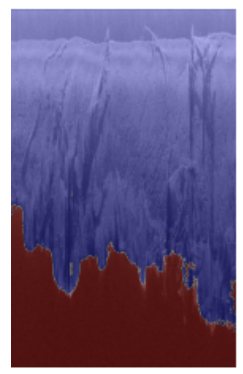

b) All filtered pixels

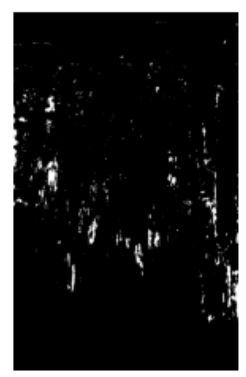

d) Filtered 1

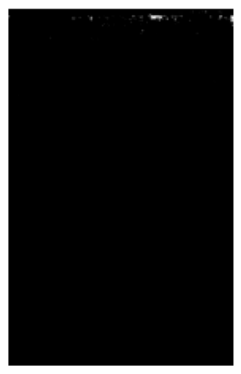

f) Filtered 2

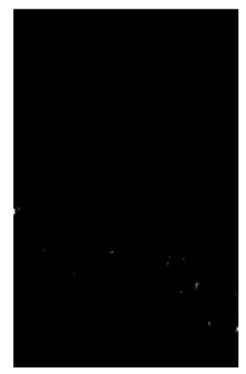

g) Class 3

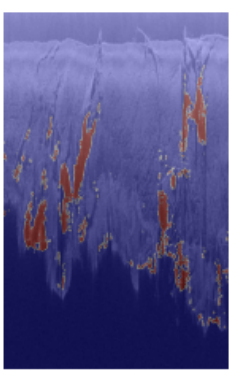

i) Class 4

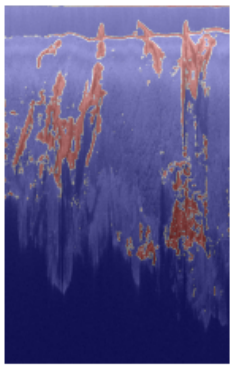

k) Class 7

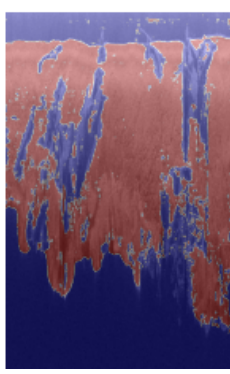

h) Filtered 3

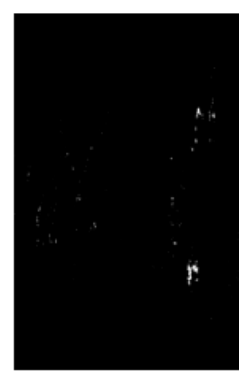

j) Filtered 4

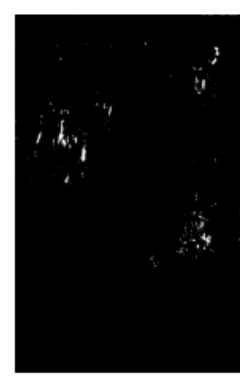

I) Filtered 7

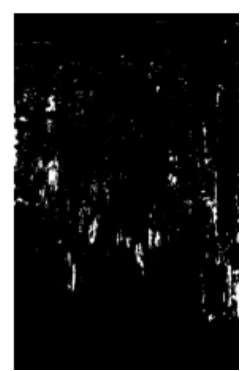

Figure 26: An illustration of the spatial filtering of the one-hot encoded labels, using an example from the sidescan dataset (a). The pixelwise prediction of five classes (called 1, 2, 3, 4, and 7) are shown in c), e), g), i) and k), and the corresponding pixels that are flagged and removed from that 2D binary pixel class map are shown alongside in, respectively, subplots d), f), h), j), and l). b) shows all pixels that have been filtered. The number of pixels flagged is not proportional to the number of overall pixels in that class. Instead, more pixels are flagged if the class is composed of smaller, more spatially isolated regions more indicative of noise than signal in the overall label image. 
a) Image

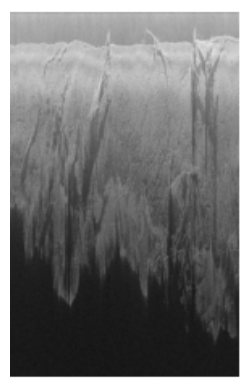

c) Class 1

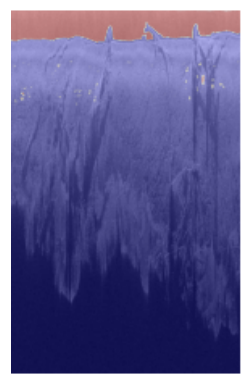

e) Class 2

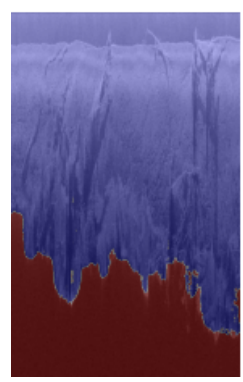

b) All filtered pixels

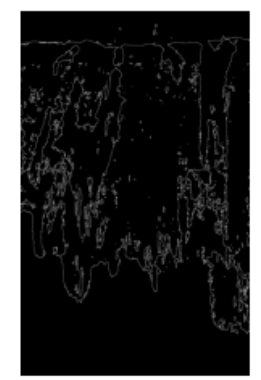

d) Filtered 1

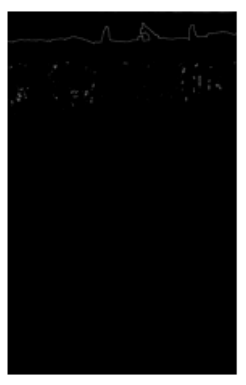

f) Filtered 2

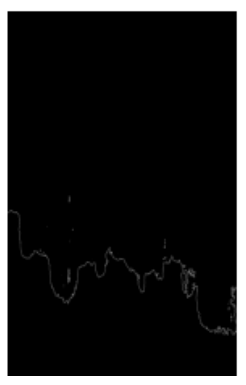

g) Class 3

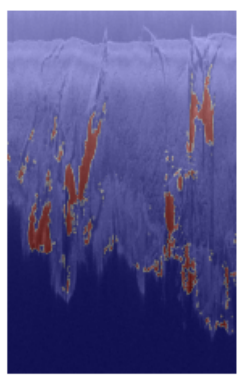

i) Class 4

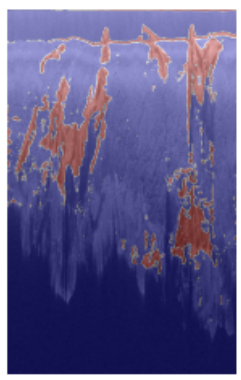

k) Class 7

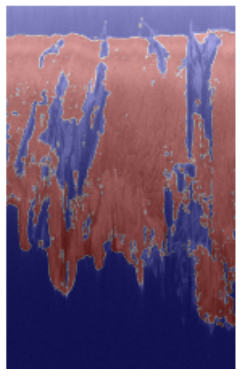

h) Filtered 3

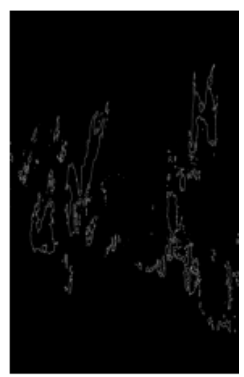

j) Filtered 4

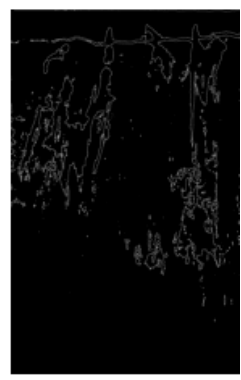

I) Filtered 7

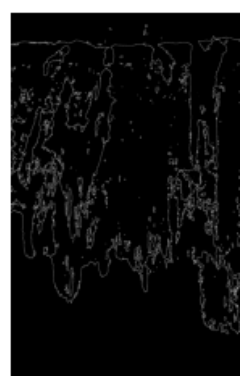

Figure 27: An illustration of the second spatial filtering procedure of the one-hot encoded labels, using a measure of distance between labeled pixels of the same class, with the same example from the sidescan dataset (a) used in Figure 26 and is structured in the same way; each of the five present classes are presented alongside a black-and-white map of pixels that have been zeroed (white), with b) a map of all pixels zeroed in this way. 
Doodler: Human-in-the-Loop Segmentation of Earth Surface Imagery

A Larsen, W Nardin, WI Van de Lageweg, and N Bätz. Biogeomorphology, quo vadis? On processes, time, and space in biogeomorphology. Earth Surface Processes and Landforms, 46(1):12-23, 2021.

Markus Reichstein, Gustau Camps-Valls, Bjorn Stevens, Martin Jung, Joachim Denzler, and Nuno Carvalhais. Deep learning and process understanding for data-driven Earth system science. Nature, 566(7743):195-204, 2019.

Evan B Goldstein, Giovanni Coco, and Nathaniel G Plant. A review of machine learning applications to coastal sediment transport and morphodynamics. Earth Science Reviews, 194:97-108, 2019.

Daniel Buscombe, Paul E Grams, and Matthew A Kaplinski. Compositional signatures in acoustic backscatter over vegetated and unvegetated mixed sand-gravel riverbeds. Journal of Geophysical Research: Earth Surface, 122(10):1771-1793, 2017.

Jie Ni, Tonghua Wu, Xiaofan Zhu, Guojie Hu, Defu Zou, Xiaodong Wu, Ren Li, Changwei Xie, Yongping Qiao, and Qiangqiang Pang. Simulation of the present and future projection of permafrost on the Qinghai-Tibet Plateau with statistical and machine learning models. Journal of Geophysical Research: Atmospheres, 126(2):e2020JD033402, 2021.

Tomas Beuzen, Evan B Goldstein, and Kristen D Splinter. Ensemble models from machine learning: An example of wave runup and coastal dune erosion. Natural Hazards and Earth System Sciences, 19(10):2295-2309, 2019.

RO Tinoco, EB Goldstein, and G Coco. A data-driven approach to develop physically sound predictors: Application to depth-averaged velocities on flows through submerged arrays of rigid cylinders. Water Resources Research, 51(2):1247-1263, 2015.

Nozomi Sugiura and Shigeki Hosoda. Machine learning technique using the signature method for automated quality control of argo profiles. Earth and Space Science, 7(9):e2019EA001019, 2020.

Nathaniel G Plant and Hilary F Stockdon. Probabilistic prediction of barrier-island response to hurricanes. Journal of Geophysical Research: Earth Surface, 117(F3), 2012.

Małgorzata Chmiel, Fabian Walter, Michaela Wenner, Zhen Zhang, Brian W McArdell, and Clement Hibert. Machine learning improves debris flow warning. Geophysical Research Letters, 48(3): e2020GL090874, 2021.

Matthew Fox, Thomas Bodin, and David L Shuster. Abrupt changes in the rate of Andean Plateau uplift from reversible jump Markov Chain Monte Carlo inversion of river profiles. Geomorphology, 238:1-14, 2015.

Evan B Goldstein and Giovanni Coco. Machine learning components in deterministic models: Hybrid synergy in the age of data. Frontiers in Environmental Science, 3:33, 2015. 
Doodler: Human-in-the-Loop Segmentation of Earth Surface Imagery

Sofia C Olhede and Patrick J Wolfe. The growing ubiquity of algorithms in society: Implications, impacts and innovations. Philosophical Transactions of the Royal Society A: Mathematical, Physical and Engineering Sciences, 376(2128):20170364, 2018.

Yolanda Gil, Cédric H David, Ibrahim Demir, Bakinam T Essawy, Robinson W Fulweiler, Jonathan L Goodall, Leif Karlstrom, Huikyo Lee, Heath J Mills, and Ji-Hyun Oh. Toward the Geoscience paper of the future: Best practices for documenting and sharing research from data to software to provenance. Earth and Space Science, 3(10):388-415, 2016.

Heng-Da Cheng, X_H_Jiang, Ying Sun, and Jingli Wang. Color image segmentation: Advances and prospects. Pattern Recognition, 34(12):2259-2281, 2001.

Mohammad D Hossain and Dongmei Chen. Segmentation for Object-Based Image Analysis (OBIA): A review of algorithms and challenges from Remote Sensing perspective. ISPRS Journal of Photogrammetry and Remote Sensing, 150:115-134, 2019.

Li Mi and Zhenzhong Chen. Superpixel-enhanced deep neural forest for remote sensing image semantic segmentation. ISPRS Journal of Photogrammetry and Remote Sensing, 159:140-152, 2020 .

AP Carleer, Olivier Debeir, and Eléonore Wolff. Assessment of very high spatial resolution satellite image segmentations. Photogrammetric Engineering \& Remote Sensing, 71(11):1285-1294, 2005.

Ioannis Kotaridis and Maria Lazaridou. Remote sensing image segmentation advances: A metaanalysis. ISPRS Journal of Photogrammetry and Remote Sensing, 173:309-322, 2021.

Stefan Lang, Geoffrey J Hay, Andrea Baraldi, Dirk Tiede, and Thomas Blaschke. GEOBIA achievements and spatial opportunities in the era of Big Earth Observation Data. ISPRS International Journal of Geo-Information, 8(11):474, 2019.

Niels S Anders, Arie C Seijmonsbergen, and Willem Bouten. Segmentation optimization and stratified object-based analysis for semi-automated geomorphological mapping. Remote Sensing of Environment, 115(12):2976-2985, 2011.

ME Gaddes, Andy Hooper, and Marco Bagnardi. Using machine learning to automatically detect volcanic unrest in a time series of interferograms. Journal of Geophysical Research: Solid Earth, 124(11):12304-12322, 2019.

Ulrike Bayr and Oskar Puschmann. Automatic detection of woody vegetation in repeat landscape photographs using a convolutional neural network. Ecological Informatics, 50:220-233, 2019. 
Doodler: Human-in-the-Loop Segmentation of Earth Surface Imagery

Hui Su, Longtao Wu, Jonathan H Jiang, Raksha Pai, Alex Liu, Albert J Zhai, Peyman Tavallali, and Mark DeMaria. Applying satellite observations of tropical cyclone internal structures to rapid intensification forecast with machine learning. Geophysical Research Letters, 47(17): e2020GL089102, 2020.

L Allan James, Michael E Hodgson, Subhajit Ghoshal, and Mary Megison Latiolais. Geomorphic change detection using historic maps and DEM differencing: The temporal dimension of geospatial analysis. Geomorphology, 137(1):181-198, 2012.

Paul E Grams, Daniel Buscombe, David J Topping, Matt Kaplinski, and Joseph E Hazel Jr. How many measurements are required to construct an accurate sand budget in a large river? Insights from analyses of signal and noise. Earth Surface Processes and Landforms, 44(1):160-178, 2019.

John Barlow, Steven Franklin, and Yvonne Martin. High spatial resolution satellite imagery, DEM derivatives, and image segmentation for the detection of mass wasting processes. Photogrammetric Engineering \& Remote Sensing, 72(6):687-692, 2006.

Lucian Drăguţ and Clemens Eisank. Automated object-based classification of topography from srtm data. Geomorphology, 141:21-33, 2012.

Justin T Ridge, Patrick C Gray, Anna E Windle, and David W Johnston. Deep Learning for coastal resource conservation: automating detection of shellfish reefs. Remote Sensing in Ecology and Conservation, 2019.

Carmen Chilson, Katherine Avery, Amy McGovern, Eli Bridge, Daniel Sheldon, and Jeffrey Kelly. Automated detection of bird roosts using NEXRAD radar data and Convolutional Neural Networks. Remote Sensing in Ecology and Conservation, 5(1):20-32, 2019.

Patrick C Gray, Abram B Fleishman, David J Klein, Matthew W McKown, Vanessa S Bézy, Kenneth J Lohmann, and David W Johnston. A Convolutional Neural Network for detecting sea turtles in drone imagery. Methods in Ecology and Evolution, 10(3):345-355, 2019.

Michael A Lefsky. A global forest canopy height map from the Moderate Resolution Imaging Spectroradiometer and the Geoscience Laser Altimeter System. Geophysical Research Letters, 37 (15), 2010.

Daniel Buscombe and Andrew C Ritchie. Landscape classification with deep neural networks. Geosciences, 8(7):244, 2018.

Patrice E Carbonneau, Stephen J Dugdale, Toby P Breckon, James T Dietrich, Mark A Fonstad, Hitoshi Miyamoto, and Amy S Woodget. Adopting deep learning methods for airborne RGB fluvial scene classification. Remote Sensing of Environment, 251:112107, 2020. 
Doodler: Human-in-the-Loop Segmentation of Earth Surface Imagery

Prem Chandra Pandey, Nikos Koutsias, George P Petropoulos, Prashant K Srivastava, and Eyal Ben Dor. Land use/land cover in view of Earth observation: Data sources, input dimensions, and classifiers-a review of the state of the art. Geocarto International, 36(9):957-988, 2021.

Ben G Weinstein. A computer vision for animal ecology. Journal of Animal Ecology, 87(3):533-545, 2018.

Priyanka Chaudhary, Stefano D’Aronco, Matthew Moy de Vitry, João P Leitão, and Jan D Wegner. Flood-water level estimation from social media images. ISPRS Annals of the Photogrammetry, Remote Sensing and Spatial Information Sciences, 4(2/W5):5-12, 2019.

John A Quinn, Marguerite M Nyhan, Celia Navarro, Davide Coluccia, Lars Bromley, and Miguel Luengo-Oroz. Humanitarian applications of Machine Learning with remote-sensing data: Review and case study in refugee settlement mapping. Philosophical Transactions of the Royal Society A: Mathematical, Physical and Engineering Sciences, 376(2128):20170363, 2018.

Travers Ching, Daniel S Himmelstein, Brett K Beaulieu-Jones, Alexandr A Kalinin, Brian T Do, Gregory P Way, Enrico Ferrero, Paul-Michael Agapow, Michael Zietz, and Michael M Hoffman. Opportunities and obstacles for Deep Learning in Biology and Medicine. Journal of The Royal Society Interface, 15(141):20170387, 2018.

Farhana Sultana, Abu Sufian, and Paramartha Dutta. Evolution of image segmentation using deep convolutional neural network: A survey. Knowledge-Based Systems, 201:106062, 2020.

Thomas Serre. Deep learning: the good, the bad, and the ugly. Annual Review of Vision Science, 5: 399-426, 2019.

Mark Everingham, Luc Van Gool, Christopher KI Williams, John Winn, and Andrew Zisserman. The PASCAL visual object classes (VOC) challenge. International Journal of Computer Vision, 88(2):303-338, 2010.

Robert Monarch. Human-in-the-Loop Machine Learning: Active learning and annotation for human-centered AI, 2021.

Karianne J Bergen, Paul A Johnson, V Maarten, and Gregory C Beroza. Machine Learning for data-driven discovery in solid Earth Geoscience. Science, 363(6433), 2019.

Renguang Zuo, Yihui Xiong, Jian Wang, and Emmanuel John M Carranza. Deep learning and its application in geochemical mapping. Earth Science Reviews, 192:1-14, 2019.

Carolina Crisci, Badih Ghattas, and Ghattas Perera. A review of supervised machine learning algorithms and their applications to ecological data. Ecological Modelling, 240:113-122, 2012. 
Doodler: Human-in-the-Loop Segmentation of Earth Surface Imagery

K Kashinath, M Mustafa, A Albert, JL Wu, C Jiang, S Esmaeilzadeh, K Azizzadenesheli, R Wang, A Chattopadhyay, and A Singh. Physics-informed Machine Learning: Case studies for weather and climate modelling. Philosophical Transactions of the Royal Society A, 379(2194):20200093, 2021a.

Daphne Koller and Nir Friedman. Probabilistic graphical models: Principles and techniques. MIT press, 2009.

E B Goldstein, D Buscombe, E Lazarus, S D Mohanty, S R Rafique, K A Anarde, A D Ashton, T Beuzen, K A Castagno, N Cohn, M P Conlin, A Ellenson, M Gillen, P A Hovenga11, J R Over, and R V Palermo. Labeling post-storm coastal imagery for machine learning: Measurement of inter-rater agreement. Earth and Space Sciences, page https://doi.org/10.1029/2021EA001896, 2021.

Christopher Bishop. Pattern Recognition and Machine Learning. Springer, January 2006. URL https://wwW.microsoft.com/en-us/research/publication/ pattern-recognition-machine-learning/.

Sanjiv Kumar and Martial Hebert. Discriminative random fields. International Journal of Computer Vision, 68(2):179-201, 2006.

Yanfei Zhong, Ji Zhao, and Liangpei Zhang. A hybrid object-oriented conditional random field classification framework for high spatial resolution remote sensing imagery. IEEE Transactions on Geoscience and Remote Sensing, 52(11):7023-7037, 2014.

George Vosselman, Maximilian Coenen, and Franz Rottensteiner. Contextual segment-based classification of airborne laser scanner data. ISPRS Journal of Photogrammetry and Remote Sensing, 128:354-371, 2017.

Philipp Krähenbühl and Vladlen Koltun. Efficient inference in fully connected CRFs with Gaussian edge potentials. Advances in Neural Information Processing Systems, 24:109-117, 2011.

Daniel Buscombe and Paul E Grams. Probabilistic substrate classification with multispectral acoustic backscatter: A comparison of discriminative and generative models. Geosciences, 8(11):395, 2018.

Jan J Koenderink and Andrea J Van Doorn. Surface shape and curvature scales. Image and Vision Computing, 10(8):557-564, 1992.

Mehmet Nadir Kurnaz, Zümray Dokur, and Tamer Ölmez. Segmentation of remote-sensing images by incremental neural network. Pattern Recognition Letters, 26(8):1096-1104, 2005.

Thomas Villmann, Erzsébet Merényi, and Barbara Hammer. Neural maps in remote sensing image analysis. Neural Networks, 16(3-4):389-403, 2003. 
Doodler: Human-in-the-Loop Segmentation of Earth Surface Imagery

Ronald Kemker, Carl Salvaggio, and Christopher Kanan. Algorithms for semantic segmentation of multispectral remote sensing imagery using deep learning. ISPRS Journal of Photogrammetry and Remote Sensing, 145:60-77, 2018.

Matt W Gardner and SR Dorling. Artificial neural networks (the multilayer perceptron) - a review of applications in the atmospheric sciences. Atmospheric Environment, 32(14-15):2627-2636, 1998.

Diederik P Kingma and Jimmy Ba. Adam: A method for stochastic optimization. Proceedings of the 3rd International Conference on Learning Representations (ICLR) arXiv:1412.6980, 2014.

Hugo Costa, Giles M Foody, and Doreen S Boyd. Supervised methods of image segmentation accuracy assessment in land cover mapping. Remote Sensing of Environment, 205:338-351, 2018.

Gabriela Csurka, Diane Larlus, Florent Perronnin, and F Meylan. What is a good evaluation measure for semantic segmentation. IEEE PAMI, 26(1), 2004.

C.R. Sherwood, J.R. Over, and K. Soenen. Structure from motion products associated with uas flights in sandwich, massachusetts. U.S. Geological Survey data release., 2021. URL https://doi.org/10.5066/P9BFD3YH.

C.J. Kranenburg, A.C. Ritchie, J.A. Brown, J.R. Over, D. Buscombe, C.R. Sherwood, J.A. Warrick, and P.A. Wernette. Post-Hurricane Florence aerial imagery: Cape Fear to Duck, North Carolina, October 6-8, 2018. U.S. Geological Survey data release, https://doi.org/10.5066/P91KB9SF., 2020.

Jin-Si R Over, Andrew C Ritchie, Christine J Kranenburg, Jenna A Brown, Daniel D Buscombe, Tom Noble, Christopher R Sherwood, Jonathan A Warrick, and Phillipe A Wernette. Processing coastal imagery with Agisoft Metashape Professional Edition, version 1.6-Structure from motion workflow documentation. Technical report, US Geological Survey, Reston, VA, USA., 2021.

Jonathan A Warrick, Andrew C Ritchie, Kevin M Schmidt, Mark E Reid, and Joshua Logan. Characterizing the catastrophic 2017 Mud Creek landslide, California, using repeat structurefrom-motion (SfM) photogrammetry. Landslides, 16(6):1201-1219, 2019.

NOAA. National Geodetic Survey Emergency response imagery. https://storms .ngs . noaa. gov/, 2021. Online; accessed May-2021.

Sean Andrew Chen, Andrew Escay, Christopher Haberland, Tessa Schneider, Valentina Staneva, and Youngjun Choe. Benchmark dataset for automatic damaged building detection from posthurricane remotely sensed imagery. arXiv preprint arXiv:1812.05581, 2018. URL https: //arxiv.org/abs/1812.05581. 
Doodler: Human-in-the-Loop Segmentation of Earth Surface Imagery

Patrick L Barnard, Jenifer E Dugan, Henry M Page, Nathan J Wood, Juliette A Finzi Hart, Daniel R Cayan, Li H Erikson, David M Hubbard, Monique R Myers, John M Melack, et al. Multiple climate change-driven tipping points for coastal systems. Scientific Reports, 11(1):1-13, 2021.

Evan B Goldstein, Somya D Mohanty, Shah Nafis Rafique, and Jamison Valentine. An active learning pipeline to detect hurricane washover in post-storm aerial images. EarthArXiv, 2020.

Daniel Buscombe. Shallow water benthic imaging and substrate characterization using recreationalgrade sidescan-sonar. Environmental modelling \& software, 89:1-18, 2017.

Daniel Buscombe, Paul E Grams, and Sean MC Smith. Automated riverbed sediment classification using low-cost sidescan sonar. Journal of Hydraulic Engineering, 142(2):06015019, 2016.

Francesco Vuolo, Mateusz Żółtak, Claudia Pipitone, Luca Zappa, Hannah Wenng, Markus Immitzer, Marie Weiss, Frederic Baret, and Clement Atzberger. Data service platform for Sentinel-2 surface reflectance and value-added products: System use and examples. Remote Sensing, 8(11):938, 2016.

Matthew J McCarthy, Kaitlyn E Colna, Mahmoud M El-Mezayen, Abdiel E Laureano-Rosario, Pablo Méndez-Lázaro, Daniel B Otis, Gerardo Toro-Farmer, Maria Vega-Rodriguez, and Frank E MullerKarger. Satellite remote sensing for coastal management: A review of successful applications. Environmental Management, 60(2):323-339, 2017.

Kilian Vos, Mitchell D Harley, Kristen D Splinter, Andrew Walker, and Ian L Turner. Beach slopes from satellite-derived shorelines. Geophysical Research Letters, 47(14):e2020GL088365, 2020.

Piotr Skalski. Make Sense. https://github.com/SkalskiP/make-sense/, 2019.

X Yao, LG Tham, and FC Dai. Landslide susceptibility mapping based on support vector machine: a case study on natural slopes of Hong Kong, China. Geomorphology, 101(4):572-582, 2008.

Floriane Provost, Clément Hibert, and J-P Malet. Automatic classification of endogenous landslide seismicity using the random forest supervised classifier. Geophysical Research Letters, 44(1): 113-120, 2017.

George LW Perry and Mark E Dickson. Using machine learning to predict geomorphic disturbance: The effects of sample size, sample prevalence, and sampling strategy. Journal of Geophysical Research: Earth Surface, 123(11):2954-2970, 2018.

Yuansheng Hua, Diego Marcos, Lichao Mou, Xiao Xiang Zhu, and Devis Tuia. Semantic segmentation of remote sensing images with sparse annotations. IEEE Geoscience and Remote Sensing Letters, 2021. 
Doodler: Human-in-the-Loop Segmentation of Earth Surface Imagery

Tsung-Wei Ke, Jyh-Jing Hwang, and Stella X Yu. Universal weakly supervised segmentation by pixel-to-segment contrastive learning. arXiv preprint arXiv:2105.00957, 2021.

Yao Wei and Shunping Ji. Scribble-based weakly supervised deep learning for road surface extraction from remote sensing images. IEEE Transactions on Geoscience and Remote Sensing, 2021.

R Stuart Geiger, Dominique Cope, Jamie Ip, Marsha Lotosh, Aayush Shah, Jenny Weng, and Rebekah Tang. "Garbage In, Garbage Out" Revisited: What Do Machine Learning Application Papers Report About Human-Labeled Training Data? Quantitative Science Studies, pages 1-32, 2021.

Lei Ding, Hao Tang, and Lorenzo Bruzzone. LANET: Local attention embedding to improve the semantic segmentation of remote sensing images. IEEE Transactions on Geoscience and Remote Sensing, 59(1):426-435, 2020.

Augusto Cunha, Axelle Pochet, Hélio Lopes, and Marcelo Gattass. Seismic fault detection in real data using transfer learning from a convolutional neural network pre-trained with synthetic seismic data. Computers \& Geosciences, 135:104344, 2020.

Xinming Wu, Luming Liang, Yunzhi Shi, and Sergey Fomel. FaultSeg3D: Using synthetic data sets to train an end-to-end convolutional neural network for 3D seismic fault segmentation. Geophysics, 84(3):IM35-IM45, 2019.

Daniel Buscombe and Roxanne J Carini. A data-driven approach to classifying wave breaking in infrared imagery. Remote Sensing, 11(7):859, 2019.

Daniel Buscombe, Roxanne J Carini, Shawn R Harrison, C Chris Chickadel, and Jonathan A Warrick. Optical wave gauging using deep neural networks. Coastal Engineering, 155:103593, 2020.

Chen Yang, Haishi Zhao, Lorenzo Bruzzone, Jon Atli Benediktsson, Yanchun Liang, Bin Liu, Xingguo Zeng, Renchu Guan, Chunlai Li, and Ziyuan Ouyang. Lunar impact crater identification and age estimation with Chang'E data by deep and transfer learning. Nature Communications, 11 (1): $1-15,2020$.

Hieu M Le, Bento Goncalves, Dimitris Samaras, and Heather Lynch. Weakly labeling the antarctic: The penguin colony case. In Proceedings of the IEEE Conference on Computer Vision and Pattern Recognition Workshops, pages 18-25, 2019.

Paul C Smits and Silvana G Dellepiane. Synthetic aperture radar image segmentation by a detail preserving Markov random field approach. IEEE Transactions on Geoscience and Remote Sensing, 35(4):844-857, 1997. 
Doodler: Human-in-the-Loop Segmentation of Earth Surface Imagery

Huawu Deng and David A Clausi. Unsupervised segmentation of synthetic aperture radar sea ice imagery using a novel Markov random field model. IEEE Transactions on Geoscience and Remote Sensing, 43(3):528-538, 2005.

Angel Bueno, Luciano Zuccarello, Alejandro Díaz-Moreno, Jack Woollam, Manuel Titos, Carmen Benítez, Isaac Álvarez, Janire Prudencio, and Silvio De Angelis. PICOSS: Python Interface for the classification of seismic signals. Computers \& Geosciences, 142:104531, 2020.

Jianghua Zhao, Xuezhi Wang, and Yuanchun Zhou. A crowdsourcing-based platform for labelling remote sensing images. In The 2020 IEEE International Geoscience and Remote Sensing Symposium, pages 3227-3230. IEEE, 2020.

Gencer Sumbul, Marcela Charfuelan, Begüm Demir, and Volker Markl. BigEarthNet: A large-scale benchmark archive for remote sensing image understanding. In The 2019 IEEE International Geoscience and Remote Sensing Symposium, pages 5901-5904. IEEE, 2019.

Karthik Kashinath, Mayur Mudigonda, Sol Kim, Lukas Kapp-Schwoerer, Andre Graubner, Ege Karaismailoglu, Leo Von Kleist, Thorsten Kurth, Annette Greiner, and Ankur Mahesh. ClimateNet: An expert-labeled open dataset and deep learning architecture for enabling high-precision analyses of extreme weather. Geoscientific Model Development, 14(1):107-124, 2021 b.

Jixuan Cai, Bo Huang, and Yimeng Song. Using multi-source geospatial big data to identify the structure of polycentric cities. Remote Sensing of Environment, 202:210-221, 2017.

Dash. A productive python framework for building web analytic applications, 2021. URL https://dash.plotly.com/introduction.

Miguel Grinberg. Flask web development: Developing web applications with python. O'Reilly Media, Inc., 2018.

Plotly. Collaborative data science, 2015. URL https://plot.ly.

React. A javascript library for building user interfaces, 2021. URL https://reactjs.org/.

Dirk Merkel. Docker: Lightweight linux containers for consistent development and deployment. Linux Journal, 2014(239):2, 2014.

Holoviz. High-level tools to simplify visualization in python, 2021. URL https://holoviz.org/ index.html,

Fabian Pedregosa, Gaël Varoquaux, Alexandre Gramfort, Vincent Michel, Bertrand Thirion, Olivier Grisel, Mathieu Blondel, Peter Prettenhofer, Ron Weiss, Vincent Dubourg, et al. Scikit-learn: Machine learning in python. the Journal of machine Learning research, 12:2825-2830, 2011. 
Charles R. Harris, K. Jarrod Millman, Stéfan J. van der Walt, Ralf Gommers, Pauli Virtanen, David Cournapeau, Eric Wieser, Julian Taylor, Sebastian Berg, Nathaniel J. Smith, Robert Kern, Matti Picus, Stephan Hoyer, Marten H. van Kerkwijk, Matthew Brett, Allan Haldane, Jaime Fernández del Río, Mark Wiebe, Pearu Peterson, Pierre Gérard-Marchant, Kevin Sheppard, Tyler Reddy, Warren Weckesser, Hameer Abbasi, Christoph Gohlke, and Travis E. Oliphant. Array programming with NumPy. Nature, 585(7825):357-362, September 2020. doi: 10.1038/s41586-020-2649-2. URL https://doi.org/10.1038/s41586-020-2649-2.

Intisar Rizwan I Haque and Jeremiah Neubert. Deep learning approaches to biomedical image segmentation. Informatics in Medicine Unlocked, 18:100297, 2020. 